\title{
Nucleosynthesis in Asymptotic Giant Branch Stars:Relevance for Galactic Enrichment and Solar System Formation
}

\author{
M. Busso, ${ }^{1}$ R. Gallino, ${ }^{2}$ and G. J. Wasserburg ${ }^{3}$ \\ ${ }^{1}$ Osservatorio Astronomico di Torino, 10025 Pino Torinese, Italy, \\ e-mail: busso@to.astro.it; ${ }^{2}$ Dipartimento di Fisica Generale, Universita'di Torino, Via P. \\ Giuria 1, 10125 Torino, Italy, e-mail: gallino@ph.unito.it; ${ }^{3}$ Lunatic Asylum, Division of \\ Geological and Planetary Sciences, California Institute of Technology, Pasadena \\ California91125,e-mail: isotopes@gps.caltech.edu
}

Key Words stellar evolution, supernovae, extinct radioactivities, $s$-process, $r$-process, red giants, low mass stars

Abstract We present a review of nucleosynthesis in AGB stars outlining the development of theoretical models and their relationship to observations. We focus on the new high resolution codes with improved opacities, which recently succeeded in accounting for the third dredge-up. This opens the possibility of understanding low luminosity C stars (enriched in $s$-elements) as the normal outcome of AGB evolution, characterized by production of ${ }^{12} \mathrm{C}$ and neutron-rich nuclei in the He intershell and by mass loss from strong stellar winds. Neutron captures in AGB stars are driven by two reactions: ${ }^{13} \mathrm{C}(\alpha, \mathrm{n}){ }^{16} \mathrm{O}$, which provides the bulk of the neutron flux at low neutron densities $\left(N_{n} \leq 10^{7} \mathrm{n} / \mathrm{cm}^{3}\right)$, and ${ }^{22} \mathrm{Ne}(\alpha, \mathrm{n})^{25} \mathrm{Mg}$, which is mildly activated at higher temperatures and mainly affects the production of $s$-nuclei depending on reaction branchings. The first reaction is now known to occur in the radiative interpulse phase, immediately below the region previously homogenized by third dredge-up. The second reaction occurs during the convective thermal pulses. The resulting nucleosynthesis phenomena are rather complex and rule out any analytical approximation (exponential distribution of neutron fluences). Nucleosynthesis in AGB stars, modeled at different metallicities, account for several observational constraints, coming from a wide spectrum of sources: evolved red giants rich in $s$-elements, unevolved stars at different metallicities, presolar grains recovered from meteorites, and the abundances of $s$-process isotopes in the solar system. In particular, a good reproduction of the solar system main component is obtained as a result of Galactic chemical evolution that mixes the outputs of AGB stars of different stellar generations, born with different metallicities and producing different patterns of $s$-process nuclei. The main solar $s$-process pattern is thus not considered to be the result of a standard archetypal $s$ process occurring in all stars. Concerning the ${ }^{13} \mathrm{C}$ neutron source, its synthesis requires penetration of small amounts of protons below the convective envelope, where they are captured by the abundant ${ }^{12} \mathrm{C}$ forming a ${ }^{13} \mathrm{C}$-rich pocket. This penetration cannot 
be modeled in current evolutionary codes, but is treated as a free parameter. Future hydrodynamical studies of time dependent mixing will be required to attack this problem. Evidence of other insufficiencies in the current mixing algorithms is common throughout the evolution of low and intermediate mass stars, as is shown by the inadequacy of stellar models in reproducing the observations of CNO isotopes in red giants and in circumstellar dust grains. These observations require some circulation of matter between the bottom of convective envelopes and regions close to the H-burning shell (cool bottom processing). AGB stars are also discussed in the light of their possible contribution to the inventory of short-lived radioactivities that were found to be alive in the early solar system. We show that the pollution of the protosolar nebula by a close-by AGB star may account for concordant abundances of ${ }^{26} \mathrm{Al},{ }^{41} \mathrm{Ca},{ }^{60} \mathrm{Fe}$, and ${ }^{107} \mathrm{Pd}$. The AGB star must have undergone a very small neutron exposure, and be of small initial mass $\left(M \leq 1.5 M_{\odot}\right)$. There is a shortage of ${ }^{26} \mathrm{Al}$ in such models, that however remains within the large uncertainties of crucial reaction rates. The net ${ }^{26} \mathrm{Al}$ production problem requires further investigation.

\section{INTRODUCTION}

This paper presents an updated view of nucleosynthesis in asymptotic giant branch (AGB) stars and of the implications for solar system composition and formation, as well as for galactic chemical history. This area of study is of growing interest and complexity owing to the confluence of results in nuclear physics, astronomical observations, laboratory studies of meteorites, and theoretical studies of AGB evolution. AGB stars show various degrees of photospheric enhancements of $\mathrm{C}$ and of elements produced by slow neutron captures (the $s$ process), in particular those elements belonging to the $\mathrm{Zr}$ and to the Ba abundance peaks. Among these red stars are the carbon stars that, because of their peculiar spectrum, were classified by Secchi (1868) as of "class four."

The definition of the $s$ process as the neutron addition mechanism running along the valley of $\beta$ stability dates back to Burbidge et al (1957) and to Cameron (1957). It occurs when the neutron density is so small that most unstable isotopes have time to decay before capturing a neutron. Since the above cited work by Burbidge et al, an extensive body of work has been dedicated to the phenomenological treatment of the $s$ process and of its signatures in the solar system abundance distribution (Clayton et al 1961, Seeger et al 1965, Clayton \& Ward 1974, Käppeler et al 1982). For an account of this research see Käppeler et al (1989) and Meyer (1994). Studying the occurrence of these processes in stars then required the development of detailed stellar models for low- to intermediate-mass stars $\left(1 \leq M / M_{\odot} \leq 10\right)$. In this work a fundamental role was played by Icko Iben and coworkers (Iben 1975, 1976, 1982, 1983; Truran \& Iben 1977; Iben \& Truran 1978, Iben \& Renzini 1982a,b; Hollowell \& Iben 1988, 1989, 1990). For extensive reviews see Iben \& Renzini 1983, Sackmann \& Boothroyd 1991, Iben 1991, and Wallerstein et al 1997.

Several observational and experimental results have contributed, in the last 15 years, to an increased interest in AGB stars. In particular, developments in high-resolution spectroscopy of evolved red giants have yielded a more precise 
understanding of their photospheric composition, where freshly synthesized materials dredged up from the interior are observed. Fundamental work on $s$-processenriched AGB stars of classes MS and S was done by Smith \& Lambert (1985, 1986). Important results were obtained also through the extensive search for Tc in evolved red giants (Dominy \& Wallerstein 1986, Wallerstein \& Dominy 1988, Little et al 1987, Little-Marenin 1989), after the original discovery of this unstable nuclide by Merrill (1952) in S stars. A more difficult but relevant issue was the attempt to analyze the composition of C stars (cf Kilston 1985, Utsumi 1985). The above studies made clear the correlation of $s$-process abundances with $\mathrm{C}$ enhancement (see e.g. Lambert 1985). Recently s-process abundances have been traced for all of the zoo of AGB stars and their descendants, from MS and S stars (Smith \& Lambert 1990) to various classes of Ba stars (Luck \& Bond 1991, North et al 1994) to the metal-poor CH giants (Vanture 1992).

In addition the observational work at long wavelengths, using improved infrared (IR) and radio techniques, has greatly increased our knowledge of circumstellar environments. From ground-based IR observations, the signatures of circumstellar grains were recognized [e.g. SiC (see Cohen 1984, Little-Marenin 1986, Martin \& Rogers 1987)]. Infrared studies received an enormous impetus from data collected by the Infrared Astronomical Satellite (IRAS), which yielded a comprehensive picture of the general evolution of dusty envelopes (see e.g. van der Veen \& Habing 1988, Willems \& de Jong 1988, Chan \& Kwok 1988, Groenewegen \& de Jong 1999). The results from the Infrared Space Observatory (ISO) mission have recently completed and extended this view (see e.g. Blommaert et al 1998, Aoki et al 1998a, 1998b; Voors et al 1998; Yamamura et al 1998; Waters et al 1998). Radio observations of molecular lines then provided information on the isotopic composition of such environments (Knapp \& Chang 1985, Jura et al 1988, Nyman et al 1993, Kahane et al 1992, Bujarrabal et al 1994a,b).

In the dusty envelopes, newly synthesized species are trapped in molecules, and some condense in dust grains before being ultimately injected into the interstellar medium (ISM). Several types of such presolar dust grains (SiC, graphite, corundum, etc) have been recovered from a wide variety of meteorites (see e.g. Huss \& Lewis 1995). A large fraction of these grains were identified as circumstellar condensates formed around AGB stars, as inferred from clear nucleosynthetic signatures (see e.g. Anders \& Zinner 1993, Ott \& Begemann 1990, Zinner 1997, Hoppe \& Ott 1997). Some graphite grains include crystals of different compounds in a regular relationship to the host grain (Bernatowicz et al 1991). This shows that dust can form in dense stellar winds, often governed by efficient grain growth in chemical equilibrium (Sharp \& Wasserburg 1995; Bernatowicz et al 1996; Lodders \& Fegley 1995, 1997; Glassgold 1996).

Finally, the existence of short-lived radioactive nuclei (especially ${ }^{26} \mathrm{Al}$ and ${ }^{41} \mathrm{Ca}$ ), in condensates of the early solar system (ESS) indicates injection from a nearby star and rapid solar system formation. This has stimulated a search for a plausible stellar source (a supernova, Wolf-Rayet, or AGB star) that might have polluted the ESS with radioactivity and perhaps triggered the collapse of the protosun in a dense molecular cloud (see e.g. Truran \& Cameron 1978; Cameron 1993; 
Wasserburg et al 1994, 1995; Cameron et al 1997; Vanhala \& Cameron 1998). Hydrodynamical models of triggered collapse and injection from such an event were presented (cf Foster \& Boss 1998, Vanhala \& Cameron 1998). An alternative hypothesis considers a local high-energy particle bombardment from the early sun during late stages of accretion (Lee et al 1999, Clayton \& Jin 1995, Glassgold et al 1998, Shu et al 1996). This mechanism may have played a role in generating some short-lived nuclei in the ESS, although there are distinct conflicts in explaining the observations (Ramaty et al 1996, Lee et al 1998). The interpretations presented in this review are based on the assumption that the observed abundances of shortlived nuclei are global solar system properties reflecting the initial composition of the protosolar cloud (SC). This approach would be subject to major changes if the early sun had played an important role in providing them.

All of the above considerations call for an interpretation based on stellar nucleosynthesis. A comprehensive review dealing with the most recent developments in AGB models and with their relevance for galactic enrichment and pollution of the ESS was therefore needed, and this need, in part, motivates this work. After a short outline of stellar evolution before the AGB phase and of previous studies on AGB stars (Sections 2 and 3), we give a rather extended account of recent calculations both for AGB evolution and for neutron capture processes (Sections 4 and 5). We show how predicted abundances of $s$-process nuclei are strongly affected by the initial metallicity and are characterized by a consistent star-to-star scatter, in which the solar $s$-process distribution is only a particular sample of a continuously changing mixture, established by galactic chemical evolution (Section 6). Concerning the problem of solar system formation, we focus our attention on the possibility that a close encounter with an AGB star may have been the source of some of the extinct radioactivity in the ESS (Section 7). For reasons of space we do not address the important constraints coming from the observed isotopic composition of circumstellar dust grains preserved in meteorites. Recent reviews on this subject can be found in Anders \& Zinner 1993, Zinner 1997, and Gallino et al 1997. The final conclusions are drawn in Section 8.

\section{STELLAR EVOLUTION PRIOR TO THE ASYMPTOTIC GIANT BRANCH PHASE}

For clarity, we discuss the phases of stellar evolution that are of relevance here, making use of a schematic view of the track followed by the stellar representative point in the $\mathrm{H}-\mathrm{R}$ diagram. We adopt models of $1-M_{\odot}$ and $5-M_{\odot}$ stars as examples (see Figure $1 a, b$ ). Core hydrogen burning starts on the zero age main sequence (ZAMS; the diagonal line in Figure $1 a, b$ ) and goes on until $\mathrm{H}$ is exhausted in the core over a mass fraction close to $10 \%$ (Iben \& Renzini 1984). Then the He core

Figure $1 a$. Schematic evolution in the H-R diagram of a $1-M_{\odot}$ stellar model and solar metallicity. All of the major evolutionary phases discussed in the text are indicated. $b$. Same as $a$, but for a stellar model of $5 M_{\odot}$ with solar metallicity. 

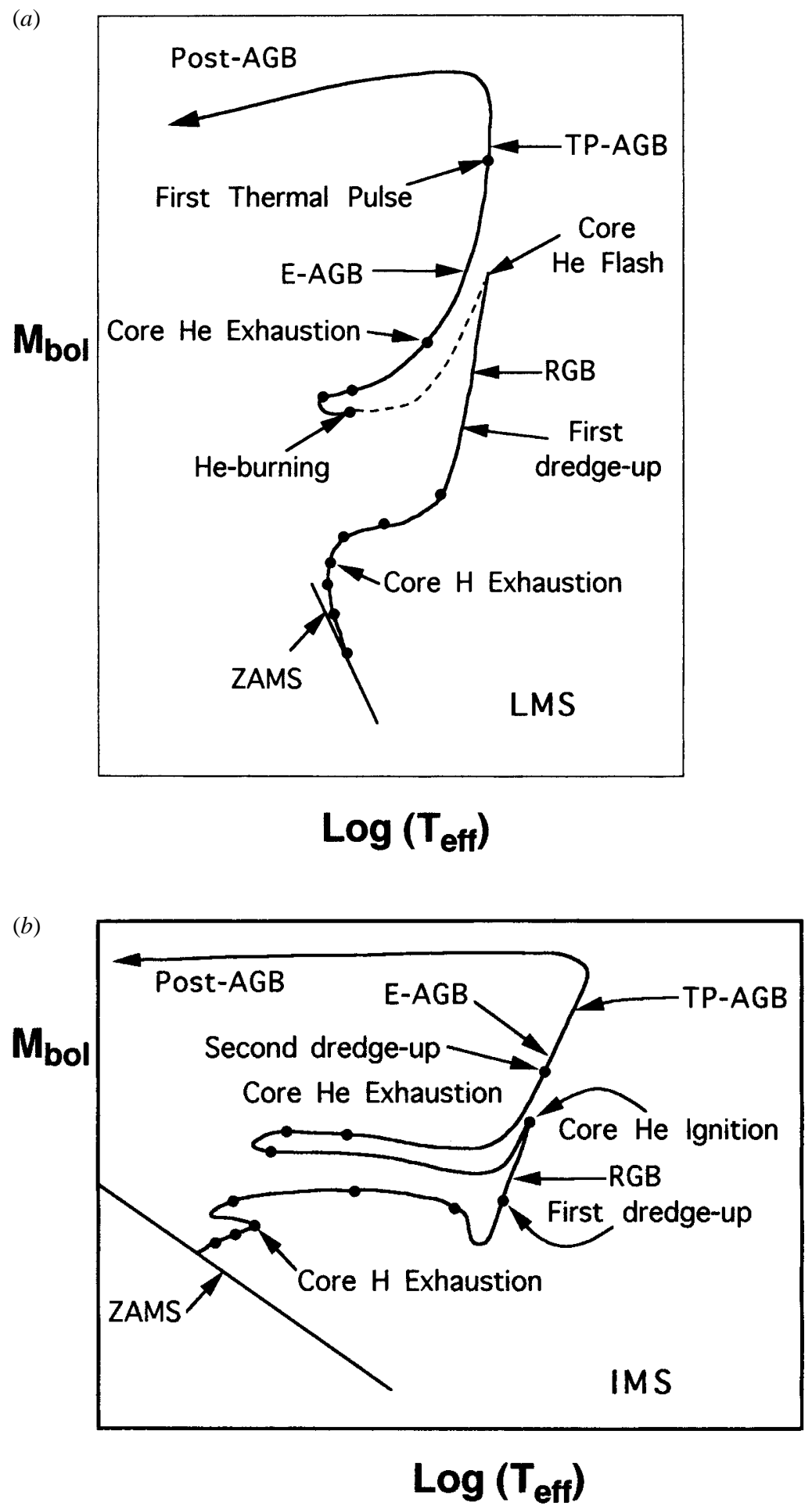
shrinks, while the stellar radius increases to carry out the energy produced by the H-burning shell (Sandage \& Schwarzschild 1952, Hoyle \& Schwarzschild 1955). As a consequence of envelope expansion, the stellar representative point in the H-R diagram moves to the red and to increasing luminosity, into the region occupied by stars with fully convective envelope structures, and then climbs a track called the red giant branch (RGB; see Figure $1 a, b$ ). The details of such an evolution have been the object of many dedicated studies over the last 20 years (e.g. Renzini 1984, Chiosi et al 1992).

While the envelope expands outward, convection penetrates into regions that, during the main sequence, had already experienced partial $\mathrm{C}-\mathrm{N}$ processing and whose abundances of light elements had therefore been changed by proton captures (Figure 2). This occurs after the star reaches the red giant stage and ascends for

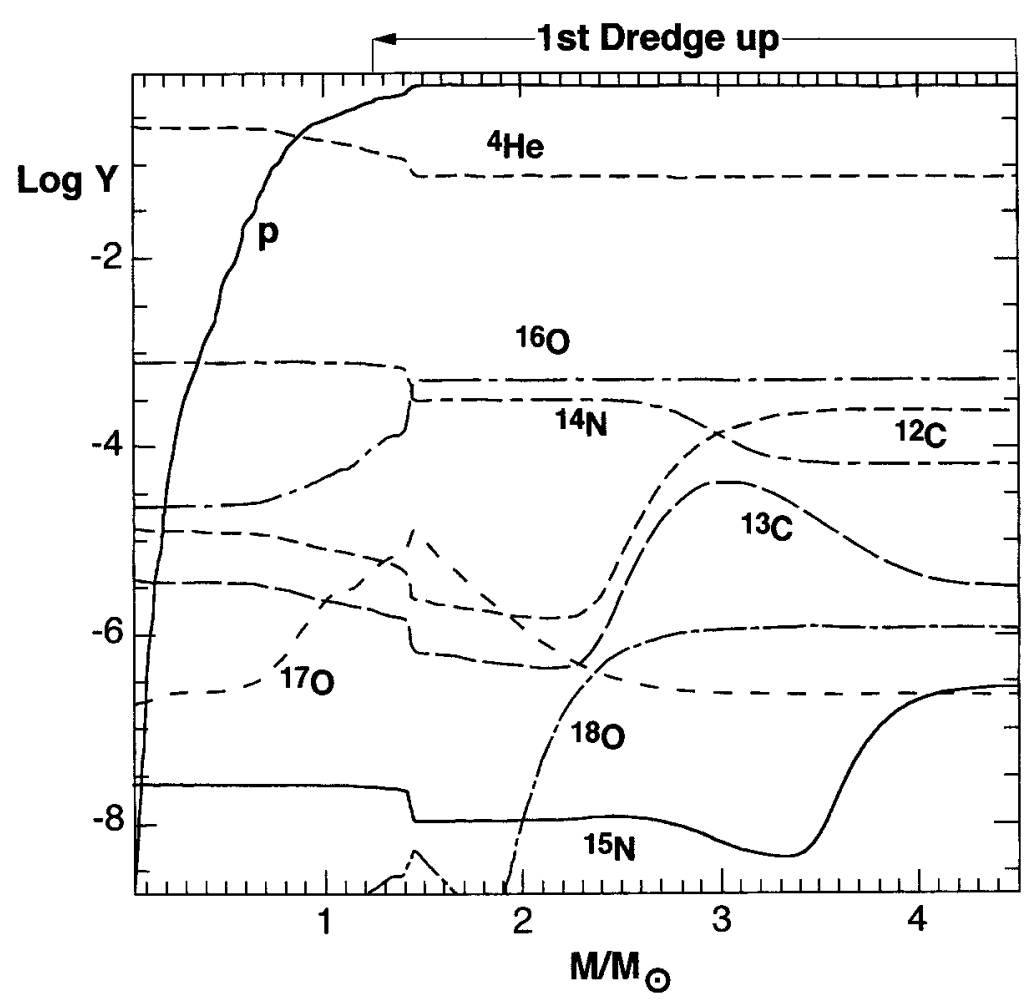

Figure 2 Distribution of the abundances of light nuclei in the inner regions of a 5- $M_{\odot}$ star with $Z=Z_{\odot}$ after core hydrogen burning. The region subsequently mixed by the first dredge-up is indicated by the arrow. Before first dredge-up, the original composition is unchanged down to $\sim 4.0 M_{\odot}$; below that region there are increases in ${ }^{13} \mathrm{C}$ and ${ }^{14} \mathrm{~N}$ and sharp decreases in ${ }^{12} \mathrm{C}$ and ${ }^{15} \mathrm{~N}$. After first dredge-up, the envelope composition is then changed to the average value mixed to the depth indicated. 


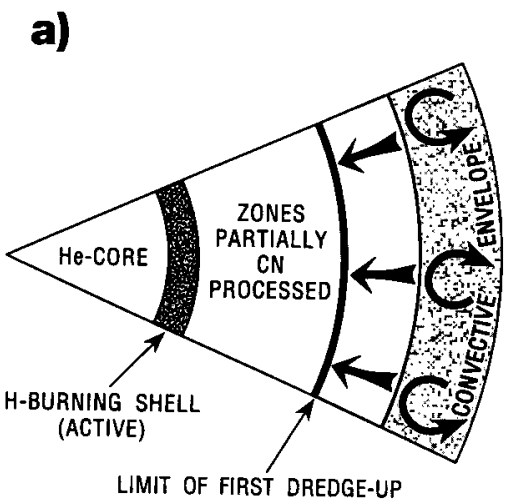

LIMIT OF FIRST DREDGE-UP

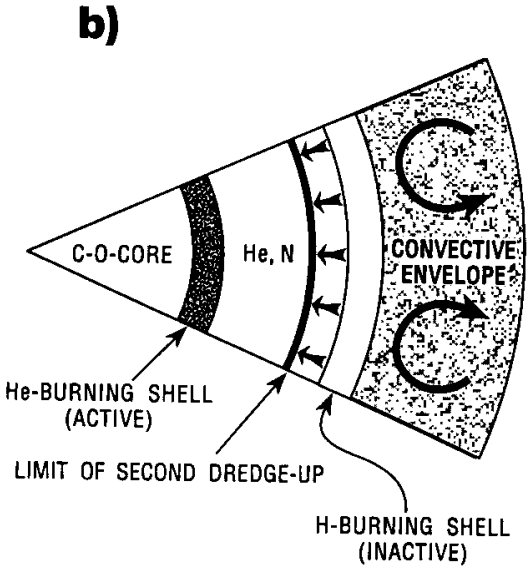

Figure $3 a$. Sketch of the internal structure of a star at the moment of first dredge-up. The energy is produced by the H-burning shell above the He core. The first dredge-up extends downward to incorporate regions where partial $\mathrm{CN}$ processing occurred during the main sequence phase and are thus incorporated into the convective envelope (see Figure 2). $b$. Second dredge-up for intermediate mass stars. Here the energy is produced by the He-burning shell during the time when $\mathrm{H}$ burning is extinguished in the $\mathrm{H}$ shell. Convection penetrates from the envelope to below the former position of the $\mathrm{H}$ shell and hence mixes with the envelope products of $\mathrm{H}$ burning.

the first time along the RGB. The ensuing process, which mixes the pre-existing layered stellar structure, homogenizing about $80 \%$ of the mass (see Figure $3 a$ ), is called the first dredge-up. In the H-R diagram the position of stars undergoing the first dredge-up is indicated in Figure $1 a$ and $b$. As a consequence, a sharp chemical discontinuity forms between the homogenized envelope and the radiative interior. The mixing modifies the surface composition: models predict a drop of $\sim 30 \%$ in ${ }^{12} \mathrm{C}$ and an increase by a factor of $2-3$ in ${ }^{13} \mathrm{C}$. The $\mathrm{N}$ and $\mathrm{O}$ isotopes are also affected: ${ }^{14} \mathrm{~N}$ increases by a factor of $3-4,{ }^{15} \mathrm{~N}$ is decreased by a factor of $\sim 2,{ }^{17} \mathrm{O}$ is enhanced by up to 1 order of magnitude, and ${ }^{18} \mathrm{O}$ is slightly depleted $(\sim 30 \%)$ (see Dearborn 1992, El Eid 1994, Lattanzio \& Boothroyd 1997). Later in their evolution, for all stars below $\sim 2.5 M_{\odot}$ the H-burning shell reaches the discontinuity in molecular weight and erases it. This does not occur in more massive stars, because the remaining evolutionary time is too short.

Observations showed that model predictions for the first dredge-up are in rough agreement with spectroscopic abundances of $\mathrm{C}$ and $\mathrm{O}$ isotopes in red giant stars of mass above $\approx 2.5 M_{\odot}$ (Lambert \& Ries 1981, Luck \& Lambert 1982, Kraft et al 1993, Kraft 1994). Below this mass limit, photospheric CNO abundances of star clusters reveal remarkable discrepancies (e.g. Gilroy 1989, Gilroy \& Brown 1991, Charbonnel 1994). Figure 4 shows a comparison of carbon and oxygen isotopic ratios predicted by stellar models with observations. The low ${ }^{12} \mathrm{C} /{ }^{13} \mathrm{C}$ ratio found 

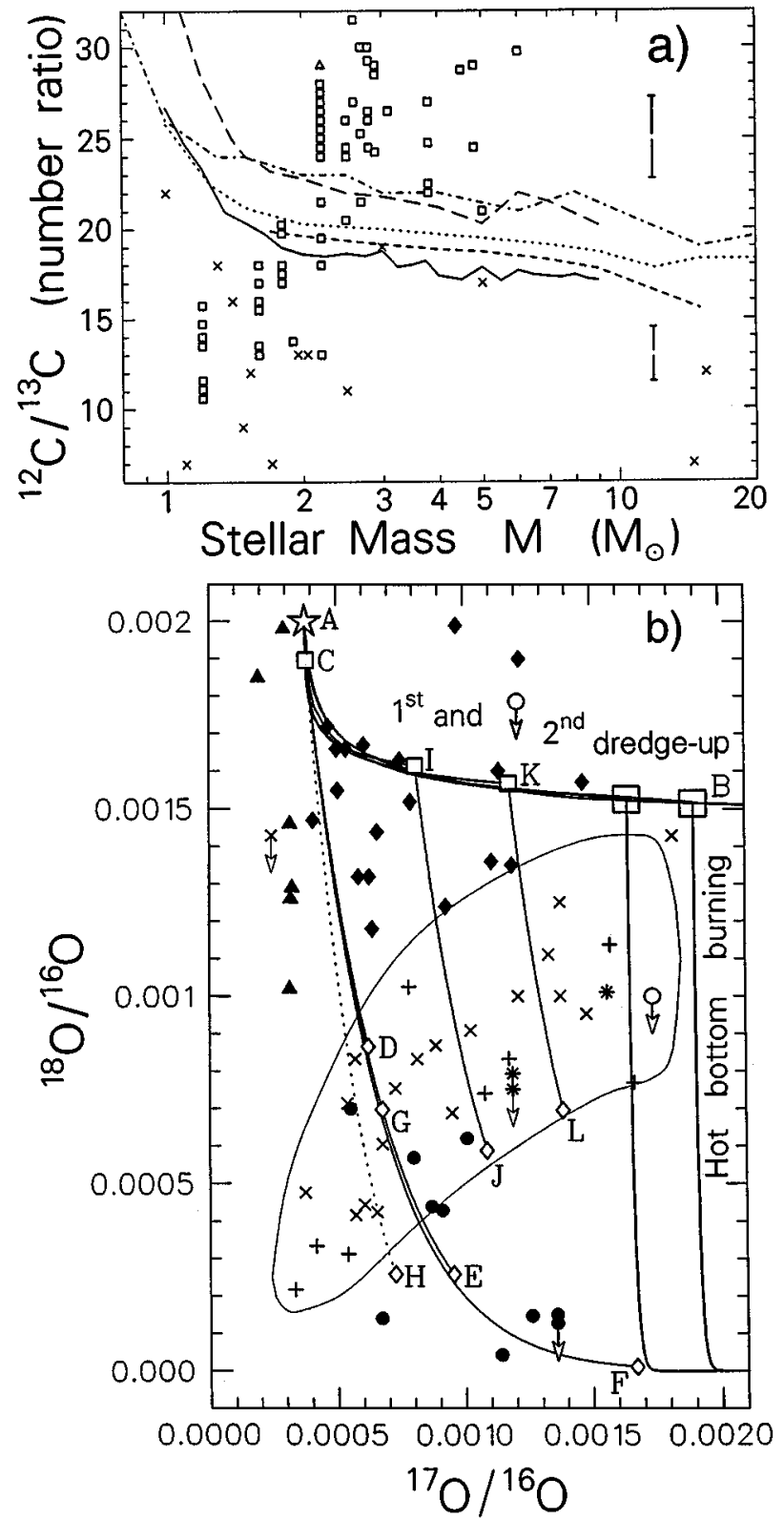
for low-mass stars approaching $\mathrm{CNO}$ equilibrium is in gross disagreement with the standard models (see Figure 4). It has also been noted (cf Pilachowski et al 1997) that giants with $M_{\mathrm{Bol}} \gtrsim 0.5 \mathrm{mag}$ consistently have a sharp drop in ${ }^{12} \mathrm{C} /{ }^{13} \mathrm{C}$. These observations appear to require an extra mixing process from the bottom of stellar envelopes to near the $\mathrm{H}$ shell to produce the extra ${ }^{13} \mathrm{C}$ and to decrease the ${ }^{18} \mathrm{O} /{ }^{16} \mathrm{O}$ ratio to the level observed in circumstellar $\mathrm{Al}_{2} \mathrm{O}_{3}$ grains and in stellar envelopes. Its nature is still much debated; it has been ascribed to turbulent diffusion, shear instabilities, or semiconvection and has sometimes been associated with rotation (Sweigart \& Mengel 1979, Zahn 1992, Charbonnel 1995, Denissenkov \& Weiss 1996, Langer et al 1999). In general, the fact that, below $2.5 M_{\odot}$, the discontinuity in molecular weight left by first dredge-up is subsequently erased by the advancing $\mathrm{H}$ shell may allow the mixing of material at the bottom of the convective envelope with regions close to the H-burning shell, where partial nuclear burning modifies the chemical composition. This process was called cool bottom processing (CBP; Boothroyd et al 1994, 1995). It appears to be independent of the details of transport so long as the extra mixing gets sufficiently close in temperature to that of the $\mathrm{H}$ shell. It was shown, from consideration of both stellar observations and data on oxygen isotopes in circumstellar grains (cf Huss et al 1994, Nittler et al 1994, Choi et al 1998) that many observational constraints can be accounted for by CBP (Boothroyd, Sackmann \& Wasserburg 1995, Wasserburg et al 1995).

For stars below a critical mass $M_{\mathrm{HeF}}\left[M_{\mathrm{HeF}}\right.$ is the mass at helium flash (see Alongi et al 1991, Bertelli et al 1985)], the conditions to ignite $\mathrm{He}$ are reached while the stellar interior is strongly electron degenerate. $M_{\mathrm{HeF}}$ spans the range from 1.7 to $2.5 M_{\odot}$, for different choices of the mixing algorithms (Shaviv \& Salpeter 1973, Maeder 1975, Sweigart \& Gross 1978, Langer 1986, Renzini 1987, Chiosi et al 1992). When $\mathrm{He}$ is ignited in degenerate conditions, a thermal runaway (core He flash) develops (Hartwick et al 1968, Buzzoni et al 1983, El Eid 1994). After a number of flashing episodes, which burn some He into C, the electron degeneracy is removed without modification of the envelope composition (Renzini \& Fusi Pecci 1988). The start of core He burning, occurring at the so-called red giant tip (Figure $1 a, b$ ), forces the representative point of the star to move off the RGB, while the H-burning shell is extinguished. A low-mass star of solar metallicity

Figure $4 a$. Comparison of the ${ }^{12} \mathrm{C} /{ }^{13} \mathrm{C}$ ratios observed in red giant stars with model predictions of first dredge-up, as a function of stellar mass. Note that stars with $M_{\odot} \leq 2 M_{\odot}$ have ${ }^{12} \mathrm{C} /{ }^{13} \mathrm{C}$ far (10-fold) below the five theoretical curves shown. Stars above this mass have ${ }^{12} \mathrm{C} /{ }^{13} \mathrm{C}$ typically within $\sim 20 \%$ of the theoretical curves shown. $b$. Comparison between observations on oxygen in $\mathrm{Al}_{2} \mathrm{O}_{3}$ grains formed as circumstellar condensates (blackened symbols) and stellar observations $(+)$. Note that the stellar observations have large errors (not shown). The trajectory $A-B$ corresponds to first and second dredge-up for standard stellar models for $Z=Z_{\odot}$. Trajectories $A, D, J, L$, and $F$ correspond to cool-bottom-processing conveyor belt calculations (after Wasserburg et al 1995) and data sources cited therein, particularly Nittler et al (1994). 
moves slightly toward the blue and to lower luminosities in the H-R diagram, to the clump region, where He burning proceeds quietly up to central He exhaustion (Figure 1a). Stars of low mass and metallicity, like those in globular clusters, in this phase populate a wider and bluer track called the horizontal branch (Faulkner 1966, Hartwick et al 1968, Castellani et al 1969). For initial masses above $M_{\mathrm{HeF}}$, electron degeneracy at core He ignition is avoided, and core He burning occurs quietly in an extended blue loop of the H-R diagram (as illustrated in Figure $1 b$ ). The rest of the evolution is similar to that of lower masses.

At core He exhaustion, the star, whose mass has been reduced by stellar winds by up to $10 \%$ (Renzini 1977, Iben \& Renzini 1984), becomes powered by He burning in a shell and partly by the release of potential energy from the gravitationally contracting C-O core. The large energy output pushes the representative point upward in the H-R diagram along a track that, for low-mass stars (see Figure 1a), asymptotically approaches the former RGB and is therefore known as the AGB. During the early phases (E-AGB; see Figure $1 a, b$ ), for all stars more massive than about $3.5 M_{\odot}$, the energy output from the He shell forces the star to expand and cool so that the $\mathrm{H}$ shell remains inactive. While on the E-AGB, all stars more massive than $\sim 3.5 M_{\odot}$ [hereafter referred to as intermediate-mass stars (IMS), while those below this limit are called low-mass stars (LMS)] experience a second mixing episode, called second dredge-up, whose position in the H-R diagram is shown in Figure $1 b$. This is driven by the expansion of the envelope, which is forced to radiate the energy produced by He burning shell and core contraction. The base of the convective envelope penetrates beyond the $\mathrm{H}$-He discontinuity (Figure $3 b$ ), mixing H-burning ashes (essentially ${ }^{4} \mathrm{He}$ and ${ }^{14} \mathrm{~N}$ ) with the envelope (Becker \& Iben 1980, Becker 1981). Note that the H-processed material dredged into the envelope is also ${ }^{15} \mathrm{~N}$ poor, so that the ratio ${ }^{14} \mathrm{~N} /{ }^{15} \mathrm{~N}$ undergoes a large increase (sixto sevenfold), and that smaller changes affect ${ }^{12} \mathrm{C}$, whereas $\mathrm{O}$ isotopes are almost unchanged (see Lattanzio \& Boothroyd 1997 for details).

\section{ASYMPTOTIC GIANT BRANCH STARS AND THE SLOW NEUTRON CAPTURE PROCESS: General Background}

\subsection{Stellar Evolution Along the Asymptotic Giant Branch}

When the E-AGB phase is terminated, the $\mathrm{H}$ shell is reignited, and from then on it dominates energy production, whereas the He shell is almost inactive, $\left(L_{\mathrm{He}} / L_{\mathrm{H}} \leq\right.$ $10^{-3}$; see Iben \& Renzini 1983). This behavior is interrupted at regular intervals by thermal instabilities of the He shell [thermal pulses (TP)]. The phenomenon was discovered by Schwarzschild \& Härm (1965) in LMS and then confirmed by Weigert (1966) in IMS. The structure of the star in this stage is schematically represented in Figures $3 b$ and 5. It is characterized by a degenerate $\mathrm{C}-\mathrm{O}$ core, by two shells (of $\mathrm{H}$ and $\mathrm{He}$ ) burning alternatively, and by an extended convective 


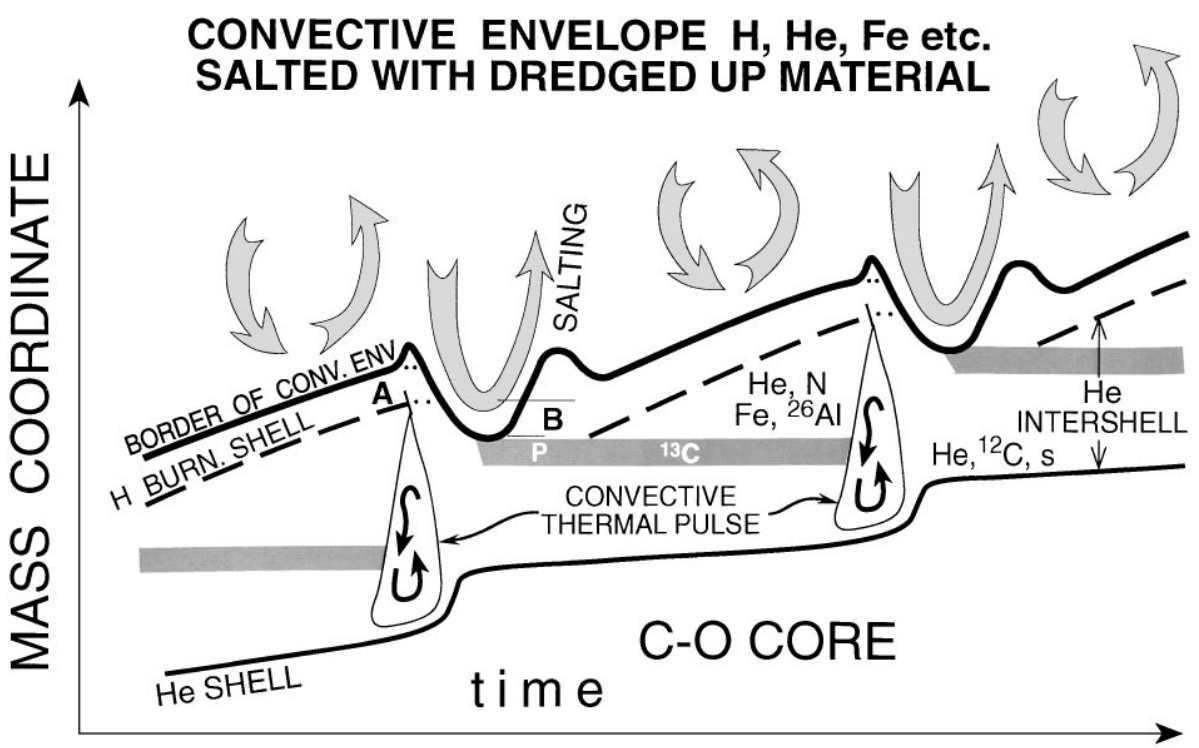

Figure 5 Illustration of the structure of a thermal pulse-asymptotic giant branch star over time, showing the border of the convective envelope, the H-burning shell, and the Heburning shell. The region between the $\mathrm{H}$ and $\mathrm{He}$ shells is the He intershell. Horizontal gray bars represent zones where protons are assumed to be ingested to make ${ }^{13} \mathrm{C}$. In the earlier models, ${ }^{13} \mathrm{C}$ was not allowed to burn until the region was engulfed in a convective pulse. In the newer models, ${ }^{13} \mathrm{C}$ is naturally burned under radiative conditions in the gray area before ingestion because of the progressive heating of the region. The slow neutron capture $(s)$ products are then engulfed by the thermal pulse, and further processing occurs owing to neutrons from the ${ }^{22} \mathrm{Ne}(\alpha, \mathrm{n})^{25} \mathrm{Mg}$ source. Region $A$ between the $\mathrm{H}$ shell and the border of the convective zone and region $B$ in the He intershell are mixed into the convective envelope during TDU, and these regions salt the envelope with freshly synthesized material. The remaining part of the He intershell region below B is also enriched in $s$-process nuclides and is partly mixed over subsequent cycles. Note that the convective thermal pulse does not reach the H-burning shell, as found by Iben (1977).

envelope that reaches dimensions of a few AU (Wallerstein \& Knapp 1998). The two shells are separated by a thin layer in radiative equilibrium that we call the $\mathrm{He}$ intershell (see Figure 5). Initially, $M_{\mathrm{H}}-M_{\mathrm{He}} \simeq 1 \times 10^{-2} M_{\odot}-2 \times 10^{-2} M_{\odot}$ for LMS, and a factor of 5-10 less for IMS. As shell $\mathrm{H}$ burning proceeds while the He shell is inactive, the mass of the He intershell $M_{\mathrm{H}}-M_{\mathrm{He}}$ increases (owing to sinking of newly formed $\mathrm{He}$ ) and attains higher densities and temperatures. This results in a dramatic increase of the He-burning rate for a short period of time, that is, in the development of a TP. The radiative state of the He intershell is thereby interrupted, and the shell then becomes almost completely convective, so that nucleosynthesis products manufactured by He burning at its bottom are carried 
close to the H-He interface (see Figure 5). Then the star readjusts its structure, expanding to radiate the energy produced by the temporarily effective He burning. This expansion pushes the $\mathrm{H}-\mathrm{He}$ interface to low temperatures, so that $\mathrm{H}$ burning is shut off, whereas the convective burning in the intershell ceases. The process is repeated many times (from $\sim 10$ to 100 s of cycles) before the envelope is completely eroded by mass loss. This evolutionary phase is usually referred to as the TP-AGB stage. It occupies the zone of the H-R diagram shown in Figure $1 a$ and $b$. The general integrated properties of stars in this phase were early studied by Paczyński $(1970,1971,1975)$ and expressed through analytical relations between key stellar parameters (luminosity, interpulse period, etc.) and the mass of the $\mathrm{H}$-exhausted core $\left(M_{\mathrm{H}}\right)$. The TP-AGB is characterized by the stellar structure shown in Figure 6

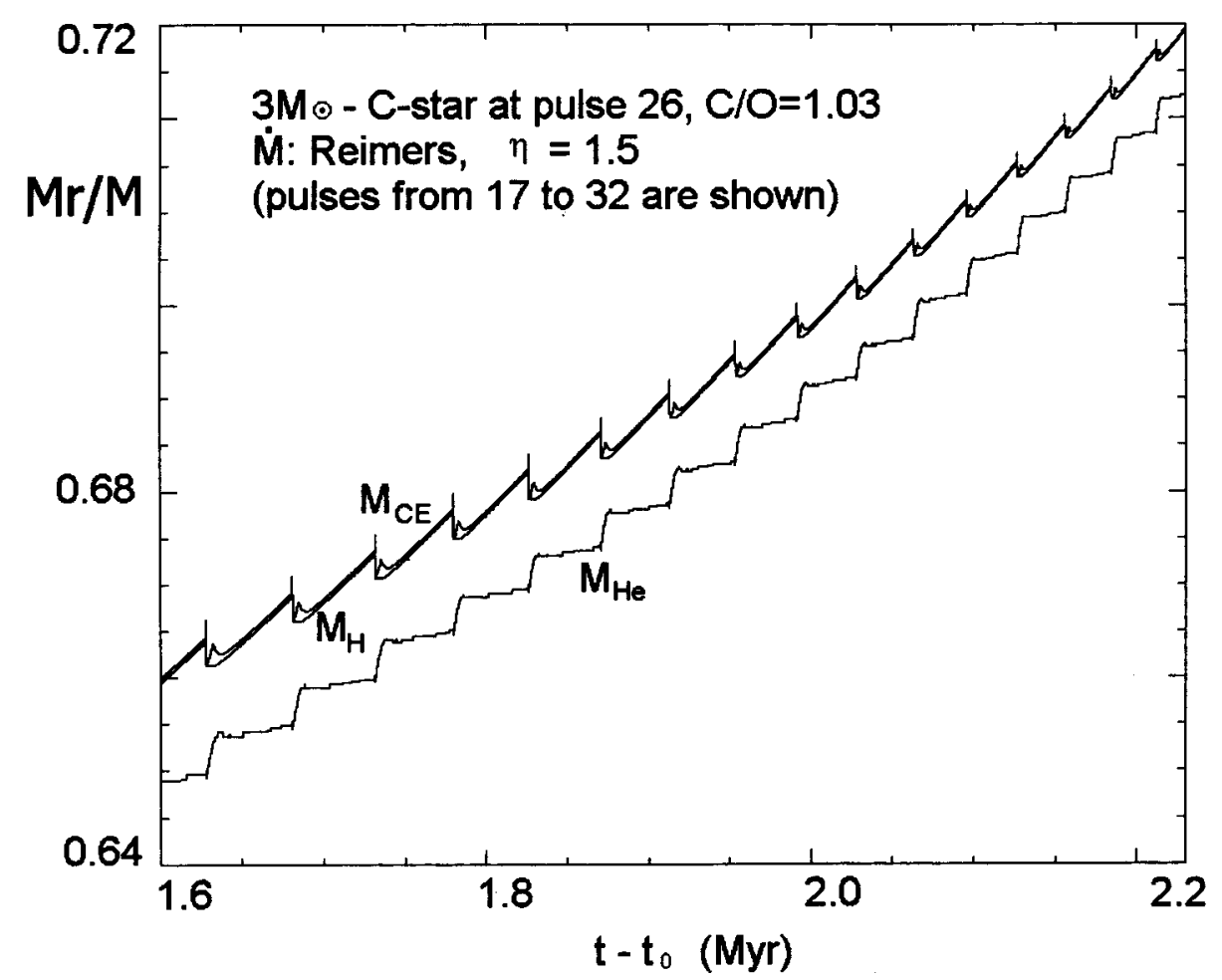

Figure 6 Plot of the internal structure of a thermal-pulse-asymptotic giant branch star as a function of time, for a 3- $M_{\odot}$ model with $Z=0.02$ (Straniero et al 1997). The positions in mass of the H-burning shell $\left(M_{\mathrm{H}}\right)$, of the He-burning shell $\left(M_{\mathrm{He}}\right)$, and of the bottom border of the convective envelope $\left(M_{C E}\right)$ are shown. Convective pulses (not shown) occupy almost the whole intershell region during the sudden advancement in mass of the He shell. Periodic penetration of the envelope into the He intershell (third dredge-up) is clearly visible. This model reaches the $\mathrm{C}$ star phase $(\mathrm{C} / \mathrm{O} \geq 1)$ at the 26th pulse. 
as a function of time, for a 3- $M_{\odot}$ model star with solar metallicity. A more complex evolution is followed by AGBs with masses in the range of 8-10 $M_{\odot}$; here the $\mathrm{C}-\mathrm{O}$ core becomes only partially degenerate, and both He- and C-thermal pulses occur (Ritossa et al 1996, Iben et al 1997, García-Berro et al 1997).

A final phase of enhanced mass loss [superwind (see Iben \& Renzini 1983)] by which the star ejects the remaining envelope mass and produces a planetary nebula, is believed to terminate the AGB evolution. As a consequence, in the post-AGB phase the stellar core (i.e. the planetary nebula nucleus) evolves first to the blue (Figure $1 a, b$ ) and then toward lower luminosities, along the cooling sequence of white dwarfs. Models for the post-AGB phases were presented by Schönberner (1979, 1983), Iben (1984), Blöcker (1995a), and Blöcker \& Schönberner (1996).

Since the first models were computed for TP-AGB stars, a critical problem has been that of understanding how freshly synthesized nuclei are mixed from the $\mathrm{He}$ intershell with the convective envelope, where they can be observed at the surface. Iben (1977) pointed out that during a thermal instability an entropy barrier exists between the He intershell and the envelope, preventing direct penetration of the convective pulse into the $\mathrm{H}$ shell. The excess entropy is later transferred outward, quenching the convective instability and causing the already mentioned sudden expansion and cooling of the envelope, whereas the inner border of envelope convection suddenly retreats. Then the system relaxes, the stellar structure shrinks again, and the bottom border of the envelope convection penetrates below the $\mathrm{H}$ $\mathrm{He}$ discontinuity, that is, below the former position of the $\mathrm{H}$ shell, now inactive. This results in a mixing process called third dredge-up (TDU), which carries some fraction of $\mathrm{H}$ - and He-burning products to the surface. From the structural point of view, the TDU is very similar to the second dredge-up in Figure $3 b$; however, its occurrence is much faster and is expected to repeat many times.

Modeling TDU was always very difficult; it was related to the choice of the opacity tables and, in the framework of the mixing-length theory, to the value of $\alpha_{P}$ (the ratio of the mixing length $l$ and the pressure scale height $H_{P}$ ). Using opacities that considered only atomic lines, the value of $\alpha_{P}$ that allowed a reproduction of the present luminosity and structure of the sun was $\alpha_{P} \sim 1-1.5$. Adopting this value, negative results on TDU models (Boothroyd \& Sackmann 1988a,b,c,d) and occasional successes (Iben 1975, Wood 1981) were both reported. Successes were sometimes favored by various specific inputs, for example, a low metallicity. TDU was found more easily in models for population-II stars or in IMS with massive envelopes (Iben 1975, Wood 1981). In most works, sufficiently high $\mathrm{C} / \mathrm{O}$ ratios to account for the formation of $\mathrm{C}$ stars could not be achieved or were obtained only for luminosities much higher than observed and/or by applying extra-mixing algorithms (Vassiliadis \& Wood 1993). Only Lattanzio $(1986,1989)$ and subsequently Straniero et al (1995), using LMS models with $\alpha_{P} \simeq 1.5$ and the Schwarzschild criterion for convection, obtained $\mathrm{C}$ stars of low enough luminosity and high enough metallicity to agree with observations. The problems in explaining self-consistently TDU and the long computational times required by this work 
caused some authors to develop semianalytical models of the TP-AGB phases, in which TDU and other model parameters were chosen to be constrained by observations (see Marigo et al 1996 and Wagenhuber \& Groenewegen 1998 for recent work in this field). The reader is referred to Wood (1981) and Iben \& Renzini (1983) for the first models with third dredge-up and to Straniero et al $(1995,1997)$ for an updated view.

\subsection{O bservational Evidence of Asymptotic Giant Branch Nucleosynthesis}

The relevance of the evolutionary phases briefly outlined above for the nucleosynthesis of heavy elements was demonstrated observationally long before any stellar model could address the problem. Indeed, after Merrill (1952) discovered that the chemically peculiar S stars, enriched in elements heavier than iron, contain the unstable isotope ${ }^{99} \mathrm{Tc}\left(\bar{\tau}=2 \times 10^{5}\right.$ years $)$ in their spectra, it was clear that ongoing nucleosynthesis occurred in situ in their interior and that the products were mixed to the surface. The fact that Tc is widespread in S stars and also in the more evolved $\mathrm{C}$ stars was subsequently confirmed by many workers on a quantitative basis (Smith \& Wallerstein 1983, Dominy \& Wallerstein 1986, Little et al 1987, Wallerstein \& Dominy 1988, Kipper 1991). Coupling of high-resolution spectroscopic observations with sophisticated stellar atmosphere models allowed the determination of heavy-element abundances in AGB stars (see Gustaffson 1989 for a discussion). In particular, Smith \& Lambert (1985, 1986, 1990) and Plez et al (1992) revealed that MS and S stars show an increased concentration of $s$-process elements. Despite large observational uncertainties, this was recognized to apply also to $\mathrm{C}$ stars, characterized by a photospheric C/O ratio above unity (Utsumi 1985, Kilston 1985, Olofsson et al 1993a,b, Busso et al 1995b; see also Figure 7). Direct information on AGB nucleosynthesis can also be derived spectroscopically from stars belonging to the post-AGB phase and evolving to the blue (see Figure $1 a, b$ ) after envelope ejection (Gonzalez \& Wallerstein 1992, Waelkens et al 1991, Decin et al 1998). Since the pioneering work of McClure et al (1980) and McClure (1984), another source of information has come from the observation of surface abundances for the binary relatives of AGB stars, that is, for the various classes of binary sources whose enhanced concentrations of n-rich elements are caused by mass transfer in a binary system (Pilachowski 1998, Wallerstein et al 1997). These involve the classical Ba stars (Luck \& Bond 1991) and their dwarf star equivalents (North et al 1994), as well as the low-metallicity CH giants (Vanture 1992). All of these works lead to the conclusion that, despite a large intrinsic scatter, an anticorrelation exists between the abundance of heavy n-rich elements and the initial metal content of the star, at least for metallicities typical of the galactic disk (see Figure 8 and Busso et al 1995b for a discussion). A summary of abundance "anomalies" of many elements was recently given by Pilachowski (1998).

From extended surveys of $\mathrm{C}$ stars and of MS and S giants in the Magellanic Clouds, an estimate of the luminosities (hence masses) could be derived. By such techniques, Blanco et al (1980) and Mould \& Reid (1987) showed that C stars 


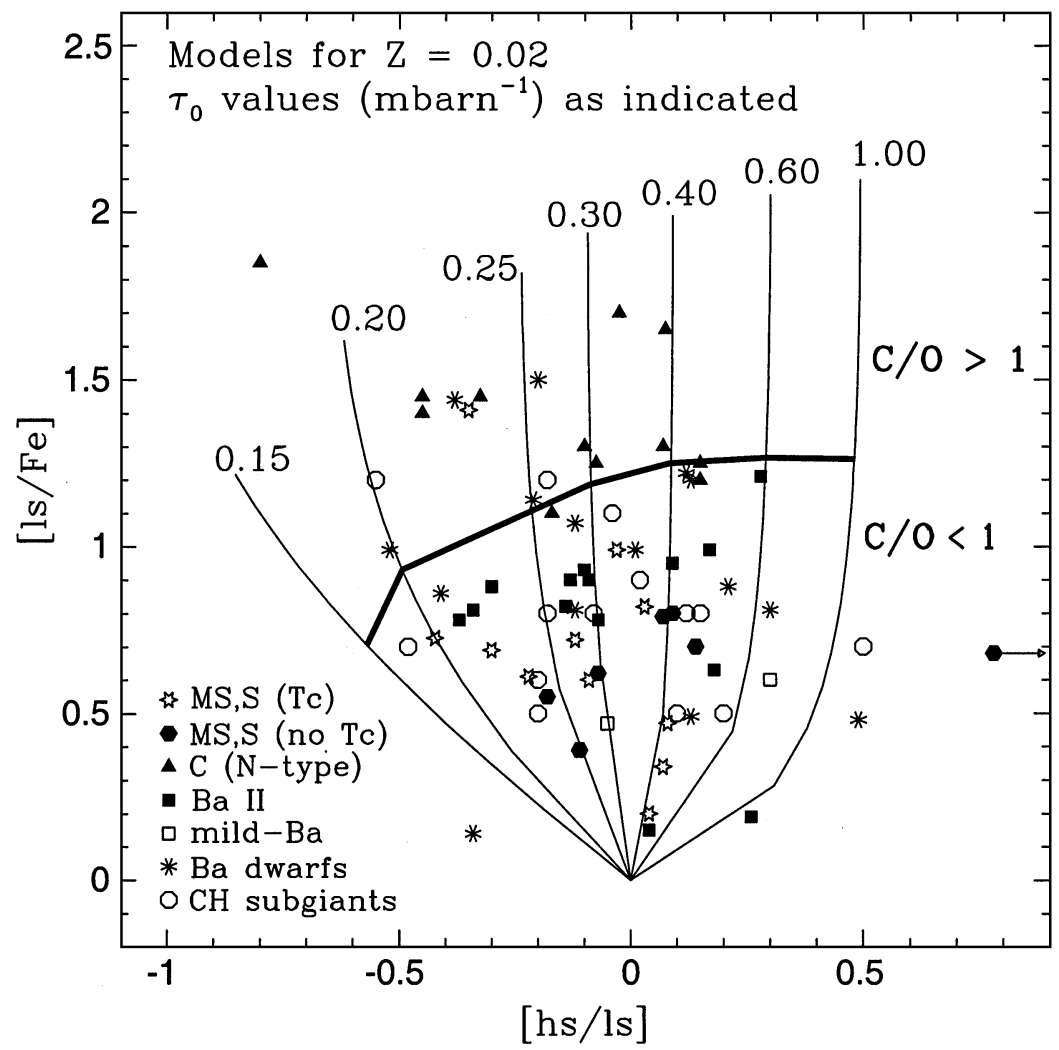

Figure 7 Observations of the logarithmic ratios [ls/Fe] of light $s$ elements (Y, $\mathrm{Zr}$ ) with respect to the logarithmic ratios between heavy (Ba, La, Nd, Sm) and light (Y, Zr) slow neutron capture $(s)$ elements. Symbols refer to different types of $s$-enriched stars. Stars with the higher $s$-element enrichments are C-rich (adapted from Busso et al 1995b). Model curves were calculated for the previous generation of $s$-process models with convective burning of ${ }^{13} \mathrm{C}$, for different values of the mean neutron exposure $\tau_{0}\left(\tau_{0}=0.28\right.$ mbarn $^{-1}$ corresponds to the solar $s$-process main component for the phenomenological model). Each curve represents the evolution of the envelope composition, for increasing dredge-up of processed material. Models with radiative burning of ${ }^{13} \mathrm{C}$ cover similar sequences when the amount of ${ }^{13} \mathrm{C}$ burnt is varied (upward or downward) within a factor of 3 compared with the standard (ST) choice of Gallino et al (1998).

are generally much fainter than the most luminous AGB stars, which appear to be O rich. This fact was confirmed more recently (see Frogel et al 1990, van Loon et al 1998, Wallerstein \& Knapp 1998). In particular, Frogel et al (1990) showed how the masses of $\mathrm{C}$ stars in Magellanic Cloud clusters do not generally exceed 2.7 $M_{\odot}$. On the other side, Smith \& Lambert (1989) and Smith et al (1995) showed that the brightest, Li-rich AGB objects are invariably of class $\mathrm{S}$, that is, O rich. For a recent review of $C$ star observations and evolution, see Wallerstein \& Knapp 


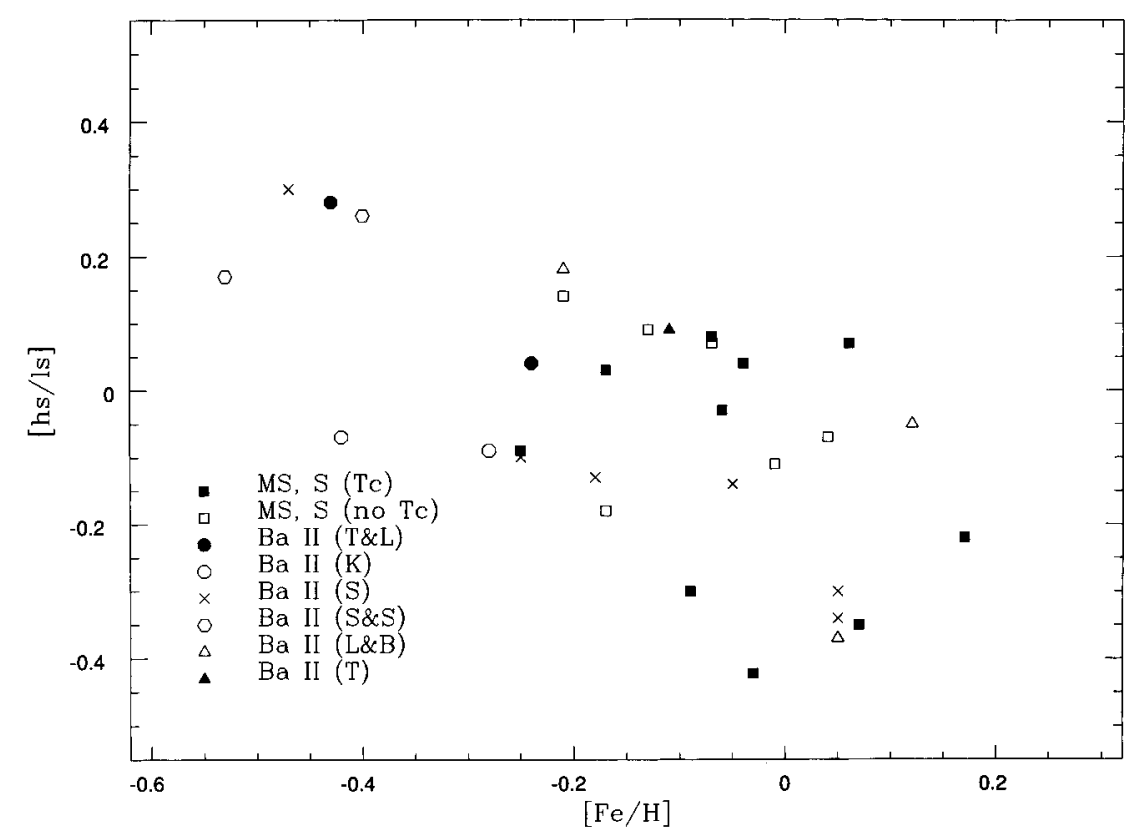

Figure 8 Observations of the logarithmic abundance ratios [hs/ls] as a function of $[\mathrm{Fe} / \mathrm{H}]$ for slow neutron capture-enriched stars in the galactic disk (symbols are as in Figure 7). On average, and despite considerable scatter, the neutron exposure (which grows with the ratio [hs/ls]) appears to increase toward low metallicities. (Adapted from Busso et al 1995b).

(1998). The above studies provided the observational basis for neutron capture nucleosynthesis studies in AGB stars, in particular for discriminating between possible neutron sources. In summary, direct observations contain compelling evidence that AGB stars are the main astrophysical site for the $s$ process and provide abundant constraints on its occurrence (e.g. its neutron exposure, correlation with ${ }^{12} \mathrm{C}$ production, inferred masses of the parent stars, etc). It is therefore not surprising that red giants in the TP-AGB phase were suggested as the site for $s$ processes as early as in the 1960s (Sanders 1967).

\subsection{The Phenomenological Approach to the Slow Neutron Capture Process}

Long before the physics of AGB stars became known in some detail, the mathematical tools for the analysis of the nucleosynthesis mechanisms controlling $s$ processes had been described by Clayton et al (1961) and by Seeger et al (1965), who pursued the original idea by Burbidge et al (1957) and built a large part of what later became known as the phenomenological approach to the $s$ process. 
They showed how considerable insight could be obtained with analytical methods. They noticed that, far from magic nuclei, the product $\sigma(A) N_{s}(A)$ of neutron capture cross-sections times $s$-process abundances is smoothly variable, so that the rule that $\sigma(A) N_{s}(A)$ is constant can be applied locally. They also recognized that, to reproduce the $s$-element abundances in the solar system, a single neutron fluence is insufficient. Defining the time-integrated neutron flux, or neutron exposure $\tau$, as $\tau=\int N_{n} v_{\mathrm{T}} d t$, where $v_{\mathrm{T}}$ is the thermal velocity and $N_{n}$ the neutron density, Seeger et al (1965) pointed out that a series of physical processes, providing a number of different values of $\tau$, were necessary to bypass the bottlenecks introduced in the neutron capture path by the very small cross-sections of the neutron magic nuclei $(N=50,82,126)$. Both a limited series of relatively large neutron exposures and a decreasing distribution of them (e.g. like an exponential form) were shown to provide a satisfactory fit to the solar system composition (see Clayton 1968). The exponential distribution soon became very popular, because it allowed a simple analytical formulation (Clayton \& Ward 1974). If $\rho(\tau) \mathrm{d} \tau$ is the number of iron nuclei in the solar system that experienced a neutron exposure between $\tau$ and $\tau+\mathrm{d} \tau$, one has $\rho(\tau)=\left(f N_{56}{ }^{\odot} / \tau_{0}\right) e^{-\tau / \tau_{0}}$, where $f\left(\equiv N_{56}{ }^{\text {capt }} / N_{56}{ }^{\odot}\right)$ is the fractional number of iron seeds that captured neutrons and $\tau_{0}$ is the mean neutron exposure. The mean neutron exposure required to reproduce the main $s$-process component in the solar system is $\tau_{0}=0.28 \mathrm{mbarn}^{-1}$ (Käppeler et al 1989, and Gallino et al 1993). For the evolution of a particular star, the value of $\tau_{0}$ depends on the metallicity, the mass, and the size of the ${ }^{13} \mathrm{C}$ pocket; hence $\tau_{0}$ for a model star may be quite different from the value for the solar fit. It was also realized that it is unlikely to obtain all the required neutron fluences in a single astrophysical site, so that three different components of the $s$ process, for the build-up of nuclei in different atomic-mass regions, were suggested: (a) the weak component, necessary for producing $s$ isotopes from $\mathrm{Fe}$ to $\mathrm{Sr} ;(b)$ the main component, for $s$ nuclei from $\mathrm{Sr}$ to $\mathrm{Pb}$; and $(c)$ a strong component, devised to provide at least $50 \%$ of ${ }^{208} \mathrm{~Pb}$. The observations discussed in the previous subsection make clear that the main component is associated with AGB stars. Each of the three components was supposed to be characterized by its own exponential distribution of exposures $\rho(\tau)$.

After the first TP-AGB models became available, Ulrich (1973) showed that, whatever the neutron source is (with the only assumption being that it burns inside the convective intershell), the recurrent mechanism of the partially overlapping TPs could easily produce an exponential distribution of neutron exposures. Indeed, the $s$-processed material is forced to undergo repeated $n$-irradiations, each followed by dilution with fresh Fe seeds. It was subsequently assumed that an exponential distribution was really required, at least for the main component, which fact, however, is not true (see Section 5).

The analyses by Seeger et al (1965) and Clayton \& Ward (1974) were followed by a long series of phenomenological studies aimed at deriving more stringent constraints on the stellar models. This approach (also known as the "classical" analysis) was rapidly developed into a technique sophisticated enough to account 


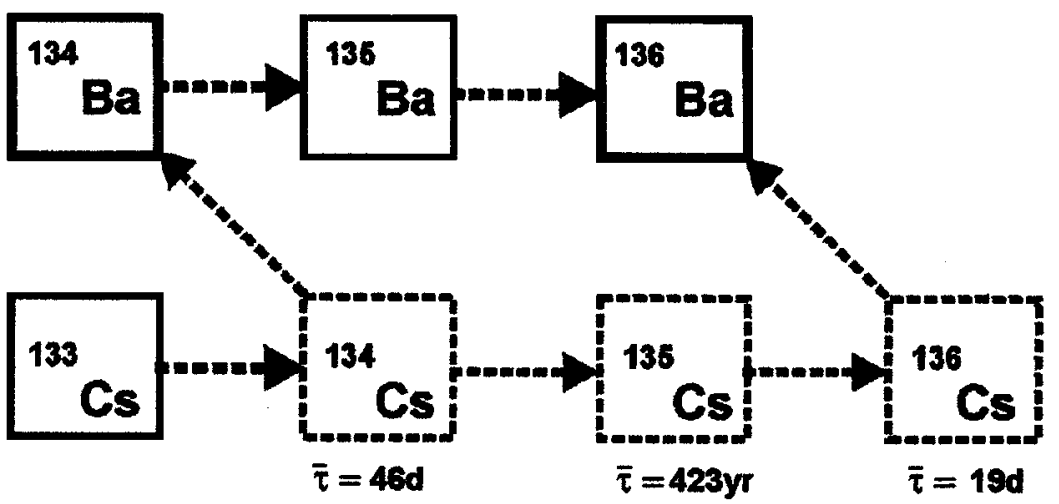

Figure 9 Neutron capture reactions branching along the slow neutron capture path at ${ }^{134} \mathrm{Cs}$ and ${ }^{136} \mathrm{Cs}$. Here the relatively long-lived ${ }^{135} \mathrm{Cs}$ may be considered as a stable isotope, as shown for simplicity. The flow to ${ }^{134} \mathrm{Ba}$ and to ${ }^{135} \mathrm{Ba}$ depends on the branch at ${ }^{134} \mathrm{Cs}$. Lifetimes of unstable nuclei are from Takahashi \& Yokoi (1987), for $T=$ $3 \times 10^{8} \mathrm{~K}$.

for reaction branchings along the $s$ path. Even at the low neutron densities characterizing the $s$ process, the competition between capture and decay still had to be considered for a number of crucial unstable isotopes (e.g. ${ }^{79} \mathrm{Se},{ }^{85} \mathrm{Kr},{ }^{148} \mathrm{Pm}$, and ${ }^{151} \mathrm{Sm}$ ). The existence of isomeric states in a number of nuclei, such as ${ }^{85} \mathrm{Kr}$, pointed in the same direction (Ward 1977). An analytical formulation of reaction branchings for a time-dependent neutron flow was given by Ward et al (1976). The branching analysis became a useful tool for deriving information on the physical parameters at the production site (average neutron density, temperature, and electron density). As an example consider Figure 9, showing the (simplified) $s$ flow through cesium and barium. Neutron captures on ${ }^{133} \mathrm{Cs}$ feed ${ }^{134} \mathrm{Cs}$; this nucleus is unstable against $\beta^{-}$decay to ${ }^{134} \mathrm{Ba}$, but its mean life is appreciable, from 0.97 years down to $46 \mathrm{~d}$ in the range $T=1 \times 10^{8}-3 \times 10^{8} \mathrm{~K}$ (see Takahashi \& Yokoi 1987). The estimated neutron capture cross-section for ${ }^{134} \mathrm{Cs}$ is large (about 1 barn at $30 \mathrm{KeV}$; see Holmes et al 1976). Hence, ${ }^{134} \mathrm{Cs}$ becomes a branching point of the reaction chain, which can produce not only ${ }^{134} \mathrm{Ba}$ but also the relatively long-lived ${ }^{135} \mathrm{Cs}$. In particular, this means that ${ }^{134} \mathrm{Ba}$ is partly bybassed, whereas ${ }^{136} \mathrm{Ba}$ is unbranched (as the two channels join on it).

For each branching, a branching ratio $f_{\beta}$ can be defined comparing the rates for $\beta^{-}$decay and neutron capture, so that $f_{\beta}=\lambda_{\beta} /\left(\lambda_{\beta}+\lambda_{n}\right)$, where $\lambda_{n}=N_{n}\langle\sigma\rangle_{b} v_{\mathrm{T}}$. Here $\langle\sigma\rangle_{b}$ is the Maxwellian averaged (n, $\gamma$ ) cross-section of the nucleus at the branching point $\left({ }^{134} \mathrm{Cs}\right.$ in the above example). Note that $\lambda_{\beta}(T)$ can be dependent on temperature and thus on the stellar model. $N_{n}$ is the neutron density and $v_{\mathrm{T}}$ is the thermal velocity. The operation of a branch sometimes controls the abundance ratio of two $s$-only isotopes (i.e. nuclei that are shielded against the $r$ process, as for the pair ${ }^{134} \mathrm{Ba}$ and $\left.{ }^{136} \mathrm{Ba}\right)$. Using the solar system $s$-process abundances, 
assuming constant neutron density, and looking for branchings at which the decay rate is independent of temperature, one can then derive $N_{n}$ from the formula $N_{n}=\left(1-f_{\beta}\right) \lambda_{\beta} /\left(f_{\beta} v_{\mathrm{T}}\langle\sigma\rangle_{b}\right)$, by choosing $f_{\beta}$ so that the abundance ratio of the two $s$ only nuclei involved is solar. The temperature can subsequently be derived by branchings in which $\lambda_{\beta}$ strongly depends on $T$, as for ${ }^{134} \mathrm{Cs}$ shown in Figure 9. It was shown that, in some cases, this procedure can also yield information on the time dependence of the neutron flux (Ward \& Newman 1978). By considering several different branchings together, the classical analysis sought to derive a self-consistent set of parameters characterizing the $s$ process. This became a guideline for stellar models, which had the task of discovering the astrophysical sites where the conditions derived a priori by the classical analysis could be fulfilled. Abundance distributions expected with an exponential form of $\rho(\tau)$ and different values of the mean neutron exposure and of the neutron density were published by Malaney (1987) and were then extensively used in the literature as approximations of what real stars should do.

The method briefly described above was continuously updated over the past two decades, to take into account progress in neutron capture cross-section measurements along the $s$ path. After the pioneering work of the Oak Ridge group led by Macklin (see e.g. Winters \& Macklin 1982 and references therein), a fundamental role in precise measurements of the key cross-sections was played by the Karlsruhe group of Franz Käppeler and coworkers (see e.g. Käppeler et al 1989, Bao \& Käppeler 1987, Beer et al 1992). They sustained the stellar s-process studies with a continuous flow of high-precision cross-section data, without which the recent improvements in the field would have been impossible. The level of accuracy reached in cross-section measurements (typically of the order of a few percent) has finally demonstrated that the phenomenological approach, based on an exponential distribution of exposures, can no longer be seen as an acceptable approximation of the $s$ process. Indeed, using the new, high-precision cross-sections, this treatment leads to large discrepancies with the solar $\sigma N_{s}$ curve for isotopes of elements like Sn, Ba, and Nd (see Käppeler 1997). Hence, the new results from updated stellar models described later (Section 5) find their correspondence in the acknowledgement that the classical analysis of $s$ processes, after its many important contributions in the past, is now superseded. A more complete account of the phenomenological approach in various stages of its development, up to the most recent works with refined cross-section results, can be found in Käppeler et al (1982), Mathews \& Ward (1985), Käppeler et al (1989), Wheeler et al (1989), Meyer (1994), and Käppeler (1997, 1999). This approach will, in any case, continue to be a key guide to nuclear astrophysical processes and problems.

\subsection{The First Models of Nucleosynthesis in Asymptotic Giant Branch Stars}

Since the works of Schwarzschild \& Härm (1967) and Sanders (1967), AGB stars have been recognized as the most promising sites for slow neutron capture 
processes, and the success of Ulrich's model (1973) gave new support to this idea. Computing detailed nucleosynthesis results for LMS and IMS thus became a very important task of stellar astrophysics. This could be done owing to improvements obtained over the years in the estimates (either theoretical or experimental) of reaction rates for charged-particle interactions in stellar interiors. This work has been carried out in laboratories dedicated to nuclear physics. For many years this field was led by William Fowler and his coworkers (e.g. see Fowler et al 1967, 1975, Caughlan et al 1985, Caughlan \& Fowler 1988, Kavanagh 1982). Several other groups made very important contributions, which unfortunately cannot be sufficiently detailed here (see reviews in Barnes 1982, Champagne \& Wiescher 1992, Rolfs et al 1987, Rolfs \& Rodney 1988). Reports on recent experimental and theoretical nuclear physics problems pertinent to this area of research can be found in the volumes of the Nuclei in the Cosmos series (e.g. see the issues edited by Käppeler \& Wisshak 1993, Busso et al 1995a, Görres et al 1997, Prantzos 1999).

In the He-burning conditions typical of the AGB phases, two neutron sources can in principle be at play: the ${ }^{13} \mathrm{C}(\alpha, \mathrm{n}){ }^{16} \mathrm{O}$ reaction, originally introduced by Cameron $(1954,1957)$ and by Greenstein (1954), and the ${ }^{22} \mathrm{Ne}(\alpha, \mathrm{n})^{25} \mathrm{Mg}$ reaction, suggested by Cameron (1960). ${ }^{22} \mathrm{Ne}$ is naturally produced in the He intershell by conversion of the original $\mathrm{CNO}$ nuclei first to ${ }^{14} \mathrm{~N}$ in the $\mathrm{H}$-burning shell and then to ${ }^{22} \mathrm{Ne}$ at the very beginning of a thermal pulse, through the reaction chain ${ }^{14} \mathrm{~N}(\alpha, \gamma){ }^{18} \mathrm{~F}\left(\beta^{+} v\right){ }^{18} \mathrm{O}(\alpha, \gamma){ }^{22} \mathrm{Ne}$. Formation of a consistent amount of ${ }^{13} \mathrm{C}$ instead needs some mixing process to bring protons into the He intershell. As mentioned (Section 3a), the direct engulfment of protons from the $\mathrm{H}$ shell (that had been suggested by Schwarzschild \& Härm 1967) was found to be impossible (Iben 1977). Alternatively, Iben (1975) showed that in IMS the reaction ${ }^{22} \mathrm{Ne}(\alpha, \mathrm{n})^{25} \mathrm{Mg}$ is naturally activated, as the temperature at the bottom of the convective intershell $\left(T_{b}\right)$ reaches values of $T_{b} \geq 3.5 \times 10^{8} \mathrm{~K}$. In this scenario, the same repeated generation of $n$ exposures envisaged by Ulrich (1973) would result, but with the ${ }^{13} \mathrm{C}(\alpha, \mathrm{n}){ }^{16} \mathrm{O}$ reaction replaced by the ${ }^{22} \mathrm{Ne}(\alpha, \mathrm{n}){ }^{25} \mathrm{Mg}$ one.

The nucleosynthesis induced by ${ }^{22} \mathrm{Ne}$ burning was analyzed by Truran $\&$ Iben (1977), who stated that an asymptotic distribution of $s$-process abundances rather similar to the solar pattern for $80 \leq A \leq 210$ could be achieved. Their result was, however, strictly related to the status of knowledge of cross-sections and solar abundances available at that time and was subsequently questioned (Mathews et al 1986). The combined information coming from detailed calculations of stellar models and from phenomenological analyses of the $s$ process soon showed that the ${ }^{22} \mathrm{Ne}(\alpha, n)^{25} \mathrm{Mg}$ source in IMS suffers from serious problems. Indeed, the peak neutron density reached in thermal pulses through ${ }^{22} \mathrm{Ne}$ burning is rather high (above $10^{11} \mathrm{~cm}^{-3}$ for $T \geq 3.5 \times 10^{8} \mathrm{~K}$ ). This implies an $n$-irradiation intermediate between the $s$ and the $r$ process (Despain 1980), leading to a reaction network that becomes rather complex, with many branchings open. This inevitably leads to a nonsolar distribution of $s$ isotopes and especially to excesses in the neutron-rich nuclides affected by $s$ branchings, like ${ }^{86} \mathrm{Kr},{ }^{87} \mathrm{Rb}$, and ${ }^{96} \mathrm{Zr}$. The possibility was envisaged that the decline of the $n$ density after its peak value could overcome 
this difficulty, because the abundances of several isotopes on the $s$-path freeze out along the $n$-density tail (Cosner et al 1980). However, the final drop in neutron density is too rapid, and it was also shown that the neutron exposure that can be achieved with the ${ }^{22} \mathrm{Ne}$ source is insufficient to produce enough heavy $s$-isotopes to account for the solar system main component (Howard et al 1986, Mathews et al 1986, Busso et al 1988).

\section{EVOLUTION AND NUCLEOSYNTHESIS IN LOW-MASS ASYMPTOTIC GIANT BRANCH STARS}

\subsection{The First Models for Low-Mass-Star Nucleosynthesis}

The stellar models so far discussed and the works on neutron captures by the ${ }^{22} \mathrm{Ne}$ neutron source were increasingly difficult to apply in explaining the observed characteristics of $\mathrm{C}$ stars. The main problems can be summarized as follows: $(a)$ the luminosities obtained by the models were far in excess of the observations for $\mathrm{C}$ stars; $(b)$ there had been great difficulty in obtaining TDU; and $(c)$ there was no well-developed technique to treat mass loss in a physically reliable way, to account for both the low- and the high-mass loss phases observed in AGB stars (this latter problem remains a fundamental issue today). Another difficulty was that stars more massive than 5-6 $M_{\odot}$ were found to experience partial $\mathrm{H}$ burning at the base of the convective envelope [hot bottom burning (HBB); see Sugimoto 1971, Scalo \& Ulrich 1973, Iben 1973, Renzini \& Voli 1981, Blöcker \& Schönberner 1991, Sackmann \& Boothroyd 1992, Vassiliadis \& Wood 1993, D’ Antona \& Mazzittelli 1996]. Proton captures in HBB partially convert carbon to ${ }^{14} \mathrm{~N}$. This prevents the formation of a $\mathrm{C}$ star unless third dredge-up (TDU) can continue to operate after HBB ceases. For example, recent calculations by Frost et al (1998) found that heavy-mass loss switches HBB off before the end of TDU. In this case, the last pulses can bring the $\mathrm{C} / \mathrm{O}$ ratio above unity. In any case, the formation of a $\mathrm{C}$ star is at least delayed and may produce ${ }^{12} \mathrm{C} /{ }^{13} \mathrm{C}$ ratios lower than those observed in normal, C- (N-type) stars. Furthermore, a series of dedicated spectroscopic observations of $s$-enriched stars did not find any sign of the expected excesses in ${ }^{25} \mathrm{Mg}$, which would result by the activation of the ${ }^{22} \mathrm{Ne}(\alpha, \mathrm{n})^{25} \mathrm{Mg}$ source. Indeed Clegg et al (1979), Smith \& Lambert (1986), and McWilliam \& Lambert (1988) found that the isotopic mix of $\mathrm{Mg}$ deduced for such cool stars by the $\mathrm{MgH}$ molecular lines is essentially solar. In addition, current estimates for the masses of $\mathrm{C}$ stars suggested that they were of low mass (Frogel et al 1990). For these reasons, while the evolution of massive AGB stars remained a fascinating and open research field, it was gradually recognized that the major role in $s$ processing and $\mathrm{C}$ production had to be played by LMS.

The maximum temperature achieved in LMS at the bottom of TPs does not exceed $T_{b}=3 \times 10^{8} \mathrm{~K}$, hence ${ }^{22} \mathrm{Ne}$ is only marginally consumed. This fact pushed some authors to reanalyze the conditions for the activation of the alternative 
${ }^{13} \mathrm{C}(\alpha, \mathrm{n}){ }^{16} \mathrm{O}$ source that had been previously abandoned. The main difficulty concerning this reaction is that of finding some mixing mechanism (other than the direct engulfment of a small amount of hydrogen from the envelope into a convective pulse), to bring protons into the intershell region, where they are subsequently captured by the abundant ${ }^{12} \mathrm{C}$ at $\mathrm{H}$ reignition, producing ${ }^{13} \mathrm{C}$.

Iben \& Renzini (1982a,b) suggested that a suitable mechanism may operate in LMS of low metallicity. Immediately after the occurrence of each thermal instability, in the cooling and expansion phase, the C-rich material is pushed into a low-temperature regime, and the local opacity strongly increases owing to partial $\mathrm{C}$ recombination. It was proposed that this might allow the formation of a semiconvective layer in which a small amount of hydrogen is dredged down into carbon-enriched zones. The entropy barrier problem discussed by Iben (1977) would thus be overcome. When the region heats up again, the reactions ${ }^{12} \mathrm{C}(p, \gamma){ }^{13} \mathrm{~N}\left(\beta^{+} v\right){ }^{13} \mathrm{C}(p, \gamma){ }^{14} \mathrm{~N}$ consume all the hydrogen, producing a ${ }^{13} \mathrm{C}$ (and ${ }^{14} \mathrm{~N}$ ) pocket of a few $10^{-4} M_{\odot}$. Iben \& Renzini (1982a,b) suggested that this pocket is engulfed by the next convective pulse, where ${ }^{13} \mathrm{C}$ nuclei easily suffer $\alpha$ captures, releasing neutrons. It was immediately clear that the effectiveness of the above mechanism would critically depend on the complex physics of convective boundaries and on the treatment of opacities in the recombination zone. However, Boothroyd \& Sackmann (1988a) did not find semiconvection to be important in their LMS models, and Iben (1983) demonstrated that it is ineffective in population-I stars. Nevertheless, for sufficiently low metallicity $(Z=0.001)$, Hollowell \& Iben (1988) confirmed the possibility of formation of a consistent ${ }^{13} \mathrm{C}$ pocket, through a local time-dependent treatment of semiconvection.

Other processes may be at play in bringing protons below the inner border of the convective envelope. In fact the TDU forces the $\mathrm{H}$-rich and the ${ }^{12} \mathrm{C}$-rich layers to establish a contact, leaving a sharp $\mathrm{H} / \mathrm{C}$ discontinuity; this is likey to be smoothed by some sort of mixing at the interface, e.g. by chemical diffusion during the interpulse phase (as considered by Iben 1982, but the effectiveness of diffusive mixing is difficult to quantify) or by rotational shear (Langer et al 1999). In the future, modeling of the proton mixing below the envelope should become the object of hydrodynamical simulations, similar to those performed for subphotospheric solar layers (see e.g. Nordlund \& Stein 1995); these should be run with inputs suitable for AGB conditions. Recently, diffusive approximations of the dynamical processes playing a role at the base of the convective envelope have started to provide encouraging results, in which a ${ }^{13} \mathrm{C}$ pocket appears to be produced (Herwig et al 1997, 1998). Fully hydrodynamical calculations have also been started, and these offer promising possibilities (Singh et al 1998). As a matter of fact, the occurrence of hydrogen penetration at the top of the ${ }^{12} \mathrm{C}$-rich intershell appears plausible, but the mass involved and the resulting ${ }^{13} \mathrm{C}$ and ${ }^{14} \mathrm{~N}$ profiles at $\mathrm{H}$ reignition have still to be treated as relatively free parameters, constrained only by the rates of proton capture on carbon isotopes. A dedicated effort on hydrodynamical grounds constitutes a major challenge for AGB calculations and $s$-process nucleosynthesis studies. 

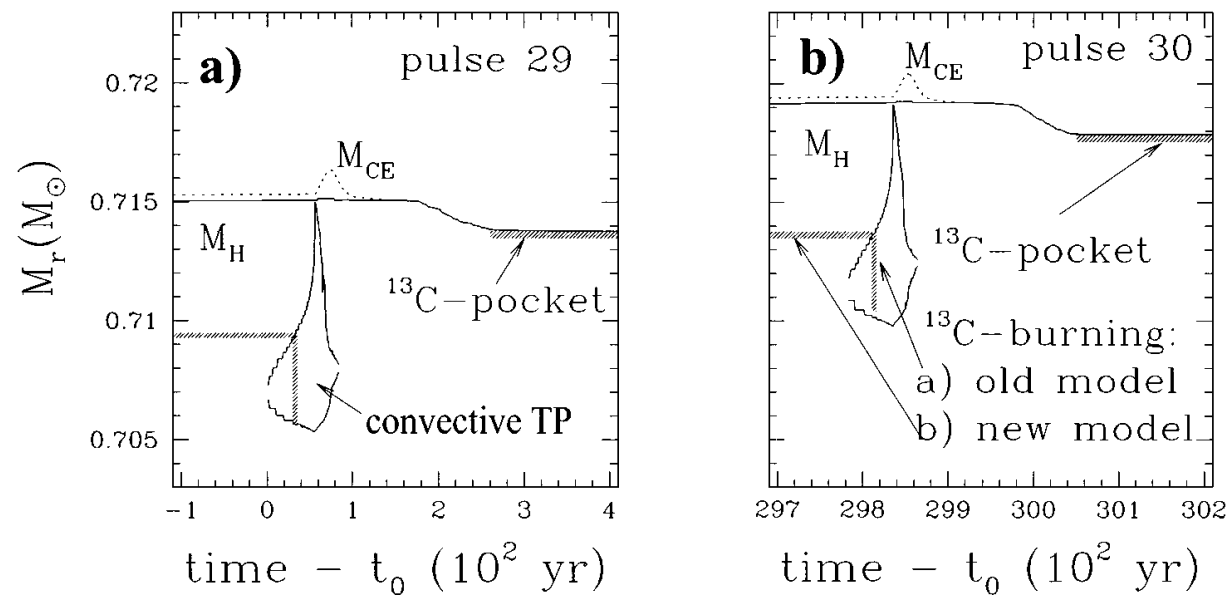

Figure 10 Two successive thermal pulses for the $3-M_{\odot}$ model with $Z=Z_{\odot}$ are shown in their relative positions as calculated from the stellar model. The shaded zone is the ${ }^{13} \mathrm{C}$ pocket, in which protons are captured by ${ }^{12} \mathrm{C}$. $a$. Ingestion and burning of ${ }^{13} \mathrm{C}$ in a pulse, based on the older models, where ${ }^{13} \mathrm{C}(\alpha, \mathrm{n}){ }^{16} \mathrm{O}$ is first burned convectively, producing the major neutron exposure, followed by a small exposure from the ${ }^{22} \mathrm{Ne}(\alpha, \mathrm{n}){ }^{25} \mathrm{Mg}$ neutron source in the pulse. $b$. The newer model in which ${ }^{13} \mathrm{C}$ burns in the thin radiative layer where it is produced, releasing neutrons locally. After ingestion into the convective intershell region, this is then followed by a second small neutron exposure from the marginal activation of the ${ }^{22} \mathrm{Ne}$ source.

A more indirect, but fruitful, approach was to study the implications of an assumed ${ }^{13} \mathrm{C}$ pocket on nucleosynthesis results, by comparing the computed abundances with observations. The first models presented with ${ }^{13} \mathrm{C}$ as the dominant neutron source were based on the hypothesis (Iben \& Renzini 1982a,b, Malaney 1986) that the ${ }^{13} \mathrm{C}$ pocket is formed and remains inactive until the occurrence of the next instability; there, it was assumed to be engulfed and burned in convective conditions. Figure 10 illustrates the development of two subsequent pulses (the 29th and 30th) for a 3- $M_{\odot}$ star model in which TDU was successfully achieved. The area marked "convective thermal pulse" represents the region swept by the pulse. Figure $10 a$ shows the position of the ${ }^{13} \mathrm{C}$ pocket before the 29 th pulse, its ingestion into the 29th pulse, and the position of the ${ }^{13} \mathrm{C}$ pocket after the pulse. The vertical line represents the ingestion and mixing of the ${ }^{13} \mathrm{C}$ pocket into the convective pulse where it is burned following the standard old model. Figure $10 b$ shows the position of the ${ }^{13} \mathrm{C}$ pocket after pulse 29 and the position after pulse 30 . In the new model, the ${ }^{13} \mathrm{C}$ pocket is consumed under radiative, not convective conditions (i.e., along the line marked ${ }^{13} \mathrm{C}$ pocket). The products are then mixed into the convective thermal pulse after ${ }^{13} \mathrm{C}$ consumption and processed further by neutrons from the ${ }^{22} \mathrm{Ne}$ source (see Section 5). When convection in the He intershell reaches its maximum extent and the bottom temperature increases to $T_{b} \sim 3 \times 10^{8} \mathrm{~K}$, the 
${ }^{22} \mathrm{Ne}$ source is marginally activated, providing a second small neutron burst of relatively high peak $N_{n}$. This neutron burst was recognized to account for several details of the solar $s$-process abundance distribution, mainly for nuclei dependent on branchings, for which the phenomenological approach suggested a relatively high temperature (Käppeler et al 1989, 1990).

Based on this approach and the idea that the two neutron sources could be activated at different times in the same TP, a number of works were dedicated to understanding the conditions under which the solar system main component could be matched in low-mass TP-AGB stars (Gallino et al 1988; Hollowell \& Iben 1989, 1990; Gallino 1989). These studies were first aimed at fitting the solar system main $s$-process component inside a single, archetypal AGB star. From these results, in the early 1990s, a more physical approach began to be followed, in which the main component was considered as the natural outcome of different $s$-process distributions averaged by galactic evolution (for an early attempt see Iben \& Truran 1978). The exploration of a wide range of stellar conditions for providing a range of $s$-process distributions was also supported by new observational constraints, coming from the growing database on abundances for evolved and unevolved stars at various metallicities (see e.g. Gratton 1996 and references therein) and from the $s$-process isotopic signatures found in presolar dust grains recovered from meteorites (see e.g. Zinner 1997).

A detailed comparison between the stellar conditions suitable for ${ }^{13} \mathrm{C}$ burning and the results from the phenomenological approach was presented by Käppeler et al (1990). This comparison showed for the first time a remarkable general agreement, in sharp contrast with the many problems previously encountered with the ${ }^{22} \mathrm{Ne}$ source. The need for averaging stellar contributions from different generations of stars was clearly stated, but a best-fit solution was also found to be possible inside individual AGB models slightly less metal rich than the $\operatorname{Sun}\left(Z_{\odot} / 3\right)$. Despite this success, the idea of ${ }^{13} \mathrm{C}$ burning in the convective pulses was also shown to have some drawbacks. In general, it provided values of $N_{n}=4 \times 10^{8}-10 \times 10^{8} \mathrm{n} \mathrm{cm}^{-3}$, higher than previously expected, unless very slow ingestion rates were assumed. Moreover, Bazan \& Lattanzio (1993) pointed out that there could be a risk of producing excessive energy by ${ }^{13} \mathrm{C}$ burning, thus modifying the pulse structure in a direction that would increase $N_{n}$ up to values incompatible with the $s$ process.

\subsection{Recent Improvements: the Formation of Low-Mass C Stars}

The field has recently gone through a phase of rapid change. In part this has been caused by the advent of new opacity tables, including contributions from molecules (Kurucz 1991, Alexander \& Ferguson 1994, Rogers \& Iglesias 1992, Sharp 1992), with large effects on the energy transfer in stellar envelopes. As discussed in detail by Sackmann \& Boothroyd (1992), the match of the solar structure with these increased opacities required values for the parameter $\alpha_{P}$ of $\sim 2-2.2$. When applied to the AGB phase, this choice has major consequences. In particular, 
owing to the inverse dependence of the stellar radius on $\alpha_{P}$, new stellar models provided more compact envelopes, which made TDU occur more easily. The new opacities stimulated a number of new calculations of AGB models by various groups (Vassiliadis \& Wood 1993, Straniero et al 1995, Forestini \& Charbonnel 1997, Frost et al 1998). However, despite these improvements, a general consensus on TDU was not achieved. Some of the new models found third dredge-up, but this was not established as a self-consistent process agreed on by all researchers. The complexities of the AGB structure, involving extreme contrasts in local material properties, the use of the mixing-length theory for describing convective transport, and the short duration of the interpulse phases available for mixing, continue to make it difficult to address the problem (Frost \& Lattanzio 1996, Lattanzio \& Boothroyd 1997).

Straniero et al $(1995,1997)$ emphasized the necessity of adopting a computational scheme with high resolution in time and mass. With opacities based only on atomic lines and using the Schwarzschild criterion for convection, they were able to obtain a self-consistent model for a 3- $M_{\odot}$ star exhibiting regular dredge-up above $M_{\mathrm{H}}=0.63 M_{\odot}$ and evolving into a C star (Straniero et al 1995). After introducing the new opacity tables requiring the increased value of $\alpha_{P}=2.1$, using their Frascati Raphson Newton Evolutionary Code (FRANEC), these workers found that TDU occurred even more efficiently. It was reported (Straniero et al 1997) that, for models of solar metallicity, formation of a $\mathrm{C}$ star occurs for $M \geq 1.5 M_{\odot}$, if no mass loss is included, and for $M \geq 2 M_{\odot}$ if mass loss is taken into account through the Reimers' formula (1975): the values of the free parameter $\eta$ were 0.7 for $M=2 M_{\odot}$ and either 1.5 or 3 for $M=3 M_{\odot}$. TDU was found to start after a limited number of pulses (when the core mass reached $M_{\mathrm{H}} \simeq 0.60 M_{\odot}$ ) and to vanish while TPs were still active, when the envelope mass was decreased below a critical limit $\left(\sim 0.5 M_{\odot}\right)$. It was stressed in these works that the time and mass resolution must be high and tuned so that variations of the physical quantities through each mass layer and through each time step remain very small and under control. These findings seem to express necessary, but not sufficient, conditions to model TDU through the Schwarzschild criterion alone. The results of other groups, using high-resolution prescriptions in different codes, sometimes confirm the above findings (Frost \& Lattanzio 1996, case C), and sometimes do not (Herwig et al 1998, Mowlavi et al 1998).

Apart from these problems, there is now general consensus on the fact that, for sufficiently low initial masses (below $\sim 1.3-1.5 M_{\odot}$ ), stellar winds should prevent the star from suffering more than a few pulses (and hence a few TDU episodes) before evolving to the blue and eventually becoming a white dwarf. They would thus not contribute much to the galactic synthesis of carbon and $s$ nuclei. In this respect, we note that AGB stars with few, if any, TDU episodes are very common. Because the initial mass function favors the lower-mass stars, we actually expect that the majority of AGBs will be of low mass and hence will not mix significant amounts of $\mathrm{C}$ and neutron-rich nuclei into the envelope. Observational evidence confirms this conclusion and shows that several AGBs, 
showing IR excesses caused by dust (including the O-rich Mira prototype $o$ Cet, $\left.M=1.2 M_{\odot}\right)$, show Tc in their atmosphere, with no other indications of $s$-process activity (Dominy \& Wallerstein 1986, Little et al 1987).

A crucial parameter for the understanding of the relevant mass range for $s$ processing is mass loss, because AGB stars are believed to be responsible for $\sim 50 \%$ of the mass return to the galaxy from evolved stars (Wallerstein \& Knapp 1998). Mass loss is currently supposed to be driven by radiation pressure on dust grains, which are consequently ejected supersonically into the ISM, dragging gas with them (Sedlmayr \& Dominik 1995). On this very important aspect of AGB evolution, stellar models have so far been essentially incapable of providing useful insights. Indeed, current laws used in modeling the mass loss rate by stellar winds are simple parameterized fits to the average mass loss rates observed [e.g. $\sim 10^{-6} M_{\odot} /$ year for C stars (Olofsson et al 1993a,b, Loup et al 1993)]. But fits to average observations obviously do not sufficiently weight the objects with the strongest winds, which predominantly contribute to the return of mass to the galaxy, in the form of both gas and dust particles (Jura 1997). They also cannot account for the extreme variability shown by mass loss rates (Jura \& Kleinmann 1989), modulated on a short time scale ( $\sim 1$ year) by stellar pulsations (Le Bertre 1998), on an intermediate time scale $(\sim 100$ years $)$ by unknown envelope instabilities (Sahai et al 1998), and on a longer time scale by the TPs (Waters et al 1994, Izumiura et al 1996). These variable mass loss phases also result in observations of detached circumstellar shells (Sahai et al 1998). A final phase of fast mass loss or "superwind" (Iben \& Renzini 1983) is also to be expected in the preplanetary nebula stage. Attempts to introduce these enhanced mass loss rates in the models have been performed (Vassiliadis \& Wood 1993, Blöcker 1995b, Schröder et al 1998), but the real situation is much too complex to be followed in a physically realistic way. The problem of the inadequate treatment of mass loss is relevant, especially because stellar winds affect the final evolution of an AGB star and the envelope composition. Strong stellar winds limit the number of pulses that a star experiences and, hence, the integrated amount of material dredged up into the envelope. Important improvements in this field are now coming from hydrodynamical models of AGB stellar winds, made by different groups (Hron et al 1997, Arndt et al 1997).

\section{RADIATIVE ${ }^{13} \mathrm{C}$ BURNING AND THE NEW SLOW-NEUTRON-CAPTURE PROCESS}

The works by Straniero et al $(1995,1997)$ represent an important step in studies of AGB evolution and nucleosynthesis, owing to the recognition that the neutron release by ${ }^{13} \mathrm{C}$ burning actually occurs in the interpulse phases, under radiative and not convective conditions. This result has been verified by various authors (Lattanzio 1995, Mowlavi et al 1996) and could have been obtained previously, if it were not for the common procedure used in past stellar models to exclude 
proton captures during He burning. Recognizing that neutrons are released by the ${ }^{13} \mathrm{C}$ source in radiative conditions makes a profound difference for all phenomenological treatments based on an exponential distribution of neutron exposures. The $s$-process abundances in AGB stars now appear to be controlled by how the hydrogen profile penetrates into the ${ }^{12} \mathrm{C}$-rich intershell; the corresponding neutron exposure is better described as a superposition of a few single irradiations (Clayton et al 1961) than as an exponential distribution. Owing to the importance of this change, we shall discuss recent results in some detail, focusing on $s$-process calculations performed by Gallino et al (1998), using the new stellar models computed by Straniero et al (1997).

In the above works it was assumed that penetration of a small amount of hydrogen below the formal envelope border occurs at each TDU episode. TDU itself was found to start after 11 pulses for a $2-M_{\odot}$ model and after 9 pulses for a $3-M_{\odot}$ Model. At $\mathrm{H}$ reignition the temperature in the $\mathrm{He}$ intershell quickly reaches values of $\sim 0.8-0.9 \times 10^{8} \mathrm{~K}$, so that the time scale $\tau_{13, \alpha}$ for $\alpha$ captures on ${ }^{13} \mathrm{C}$ becomes much smaller than the available time before the next pulse (several $10^{4}$ years for LMS). Hence, any ${ }^{13} \mathrm{C}$ left behind by partial-mixing mechanisms will burn in radiative conditions in the tiny zone $(\sim 1 / 20$ of the intershell) where it was produced, that is, the ${ }^{13} \mathrm{C}$ pocket; and only the reaction products will be ingested into the convective pulse. This avoids the risk of structural changes from the energy release by the ${ }^{13} \mathrm{C}(\alpha, \mathrm{n}){ }^{16} \mathrm{O}$ reaction (Bazan \& Lattanzio 1993). Indeed, the $\mathrm{H}$ shell is active in the star and dominates any other energy source. The rate for the ${ }^{13} \mathrm{C}(\alpha, \mathrm{n}){ }^{16} \mathrm{O}$ reaction has recently been revised on experimental grounds by Denker et al (1995), whose results were used by Gallino et al (1998). In the temperature range of interest, this rate is up to a factor of 2 higher than estimated by Caughlan \& Fowler (1988).

Figure 10 illustrates the new situation for ${ }^{13} \mathrm{C}$ burning, as compared with the previous scenario. In the present point of view, the ${ }^{13} \mathrm{C}$ pocket is the region where $s$ processing occurs under radiative conditions. The hydrogen profile adopted for these zones by Gallino et al (1998) was similar to that found by Hollowell \& Iben (1988), but was restricted to hydrogen mass fractions below $X_{\mathrm{H}} \sim 0.0015$, that is, to number ratios $N(\mathrm{H}) / N\left({ }^{12} \mathrm{C}\right)<0.1$. (A higher $\mathrm{H}$ abundance would be accompanied by a correspondingly higher abundance of ${ }^{14} \mathrm{~N}$, which is the dominant neutron absorber in these zones.) Due to the gradient in $\mathrm{H}$ and to the radiative conditions that maintain the layered structure, the concentration of ${ }^{13} \mathrm{C}$ which is locally formed when protons are captured by the ambient ${ }^{12} \mathrm{C}$ is dependent on the position. The same is true for the neutron density and the neutron exposure characterizing the $s$ process that ensues when this ${ }^{13} \mathrm{C}$ undergoes $(\alpha, \mathrm{n})$ captures. For the sake of simplicity Gallino et al (1998) modeled these continuously changing conditions using three layers. The temporal behavior of $N_{n}$ (dashed line) in the central layer of their simulation during ${ }^{13} \mathrm{C}$ consumption is shown in Figure 11a. It reaches rather low values, at most $10^{7} \mathrm{n} \mathrm{cm}^{-3}$. Consequently, only a small number of reaction branchings along the $s$ path are open during radiative ${ }^{13} \mathrm{C}$ burning. In contrast, the neutron exposure $\Delta \tau$ in the same zone is quite large $\left(\Delta \tau \sim 0.40 \mathrm{mbarn}^{-1}\right)$. The $s$ processing in each layer is highly effective; enhancement factors for the 

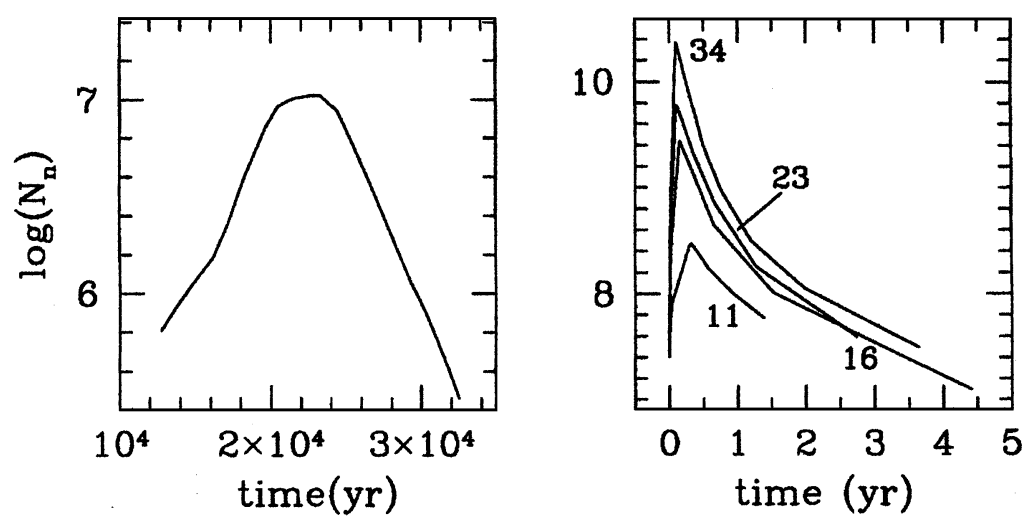

Figure 11 Neutron density during slow neutron capture processing in thermal-pulsing asymptotic giant branch stars. $a$. The situation in the central layer of the radiative zone where ${ }^{13} \mathrm{C}$ burns in the interpulse period, according to the schematic model of Gallino et al (1998). $b$. Neutron density from activation of the ${ }^{22} \mathrm{Ne}(\alpha, \mathrm{n})^{25} \mathrm{Mg}$ neutron source in different pulses. The bottom temperature increases with pulse number $(11,16$, etc), as does the neutron density.

initial composition of $s$-only isotopes range from $1 \times 10^{3}$ to $5 \times 10^{4}$. The next convective instability of the He shell mixes the $s$-process nuclei manufactured in the ${ }^{13} \mathrm{C}$ pocket over the intershell zone, so that the material over which the next neutron capture episode operates is a mixture of original Fe nuclei, of light elements (some coming from previous shell $\mathrm{H}$ burning), and of $s$-processed material left behind in the intershell at the quenching of the previous pulse. Note that the mass spanned by convective instabilities and their mutual overlap factor $r$ decrease with pulse number. As the $\mathrm{H}$ shell advances in mass into the envelope, another reservoir of ${ }^{13} \mathrm{C}$ becomes available, as nuclei present in the $\mathrm{H}$-burning ashes are added to the He-intershell. This occurs above the ${ }^{13} \mathrm{C}$ pocket, in regions that experience a lower temperature and are rich in ${ }^{14} \mathrm{~N}$. They can survive the interpulse phase and be engulfed by the next pulse. However, the ${ }^{13} \mathrm{C}$ abundance established here by $\mathrm{H}$-burning equilibrium is small and so is its contribution to $s$-process synthesis.

During late pulses, an additional neutron flux is released by the ${ }^{22} \mathrm{Ne}$ source, whose strength varies with pulse number owing to a slight increase of the maximum $T_{b}$ in the convective shell. The average $N_{n}$ caused by the ${ }^{22} \mathrm{Ne}$ source is shown in Figure $11 b$ for different thermal pulses of a $3-M_{\odot}$ model with $Z=Z_{\odot}$. The neutron density reaches a high value of a few times $10^{10} \mathrm{n} / \mathrm{cm}^{3}$. In Gallino et al (1998), the rates of the ${ }^{22} \mathrm{Ne}(\alpha, \mathrm{n})^{25} \mathrm{Mg}$ reaction and of the competing channel ${ }^{22} \mathrm{Ne}(\alpha, \gamma){ }^{26} \mathrm{Mg}$ were taken from recent experimental measurements (Käppeler et al 1994, Denker et al 1995), after excluding a possible resonance at $633 \mathrm{keV}$. In the temperature range of interest, these rates are up to a factor of 3 higher than the Caughlan \& Fowler (1988) estimates. The consequences of this second neutron irradiation on the final abundances of $s$-branched nuclei are important. They result from the high peak $N_{n}$ and from the temperature dependence of the decay rate of 
many unstable nuclei along the $s$ path. Note that the $s$-processed material retains a memory of all the previous high-temperature phases. An example of the effect of the second small ${ }^{22} \mathrm{Ne}$ neutron burst is the production of $s$-only ${ }^{164} \mathrm{Er}$, which is possible because ${ }^{163} \mathrm{Dy}$, a normally stable nuclide, becomes unstable in He shell conditions (Takahashi \& Yokoi 1987). Other examples are given by the branchingdependent $s$-only nuclei ${ }^{134} \mathrm{Ba}$ and ${ }^{152} \mathrm{Gd}$. They are actually highly overproduced during the major ${ }^{13} \mathrm{C}$ neutron exposure at low neutron density, but subsequently suffer an important depletion during the short ${ }^{22} \mathrm{Ne}$ neutron peak and are eventually restored when the neutron density falls below $\sim 10^{8} \mathrm{n} / \mathrm{cm}^{3}$ (Käppeler et al 1990). A last example is the depletion of the pair ${ }^{86,87} \mathrm{Sr}$ (which are sensitive to the branching at ${ }^{85} \mathrm{Kr}$ ), relative to the unbranched ${ }^{88} \mathrm{Sr}$ (which in contrast is mostly fed by the major neutron exposure released during ${ }^{13} \mathrm{C}$ consumption). Isotopes on the neutron-rich side of $s$ branchings generally receive a low contribution (or are partially destroyed) by the ${ }^{13} \mathrm{C}$ neutron source, because of the low neutron density, whereas they can be fed by the ${ }^{22} \mathrm{Ne}$ source when the neutron density reaches a high peak value. This is particularly true near the neutron magic zones. Characteristic isotopes are ${ }^{86} \mathrm{Kr},{ }^{87} \mathrm{Rb},{ }^{96} \mathrm{Zr}$, and also ${ }^{60} \mathrm{Fe}$, whose production is controlled by the branching at unstable ${ }^{59} \mathrm{Fe}$.

Based on the above analysis, $s$-process nucleosynthesis in an AGB star can be summarized as occurring in different phases: i) penetration of a small amount of protons into the top layers of the cool He intershell (to form a proton pocket), ii) formation of a ${ }^{13} \mathrm{C}$ pocket at $\mathrm{H}$ reignition, iii) release of neutrons by the ${ }^{13} \mathrm{C}(\alpha, \mathrm{n}){ }^{16} \mathrm{O}$ reaction when the region is subsequently compressed and heated to $T=(0.8-0.9) \times 10^{8} \mathrm{~K}$ [here $s$ processing takes place locally under radiative conditions ( $s$-enhanced pocket)], iv) ingestion into the convective thermal pulse, where the $s$-enhanced pocket is mixed with $\mathrm{H}$-burning ashes from below the $\mathrm{H}$ shell (Fe seeds, ${ }^{14} \mathrm{~N}$ ) and with $s$-processed material already from the previous pulses, v) exposure to a small neutron irradiation at high $N_{n}$ by the ${ }^{22} \mathrm{Ne}$ source over the mixed material in the pulse, vi) occurrence of the TDU episode after the quenching of the thermal instability, so that part of the $s$-processed and ${ }^{12} \mathrm{C}$-rich material is mixed into the envelope, vii) repetition of the above cycle until the TP phase is over.

\section{METALLICITY EFFECTS AND THE CHEMICAL EVOLUTION OF THE GALAXY}

\subsection{Model Predictions at Different Metallicities}

To illustrate the dependence of $s$-process predictions on metallicity, we consider as an example the results obtained with the thin ${ }^{13} \mathrm{C}$ pocket of Gallino et al (1998). This pocket was used as a standard (ST) choice by Gallino et al $(1997)\left[M\left({ }^{13} \mathrm{C}\right)=\right.$ $3 \times 10^{-6} M_{\odot}$ burnt per pulse]. We recall here that both smaller and larger values are expected in MS, S, and C stars, with a considerable star-to-star scatter, as required by the observed spreads in the $s$-process enhancements of chemically peculiar stars (Smith \& Lambert 1990, Luck \& Bond 1991, Busso et al 1995b). On galactic time scales this spread of $s$-process abundance distribution is melded by the general 
process of mixing into the ISM. Hence, only the average abundance of ${ }^{13} \mathrm{C}$ burnt at each metallicity is really relevant for studies of galactic enrichment, whereas the detailed structure of the ${ }^{13} \mathrm{C}$ pocket plays a role in explaining the abundances of individual stars.

For a given choice of the ${ }^{13} \mathrm{C}$ pocket, the efficiency of $s$ processing turns out to be strongly dependent on metallicity. Under the assumptions discussed above, the results are shown in Figure 12, in terms of the logarithm of the yields of crucial

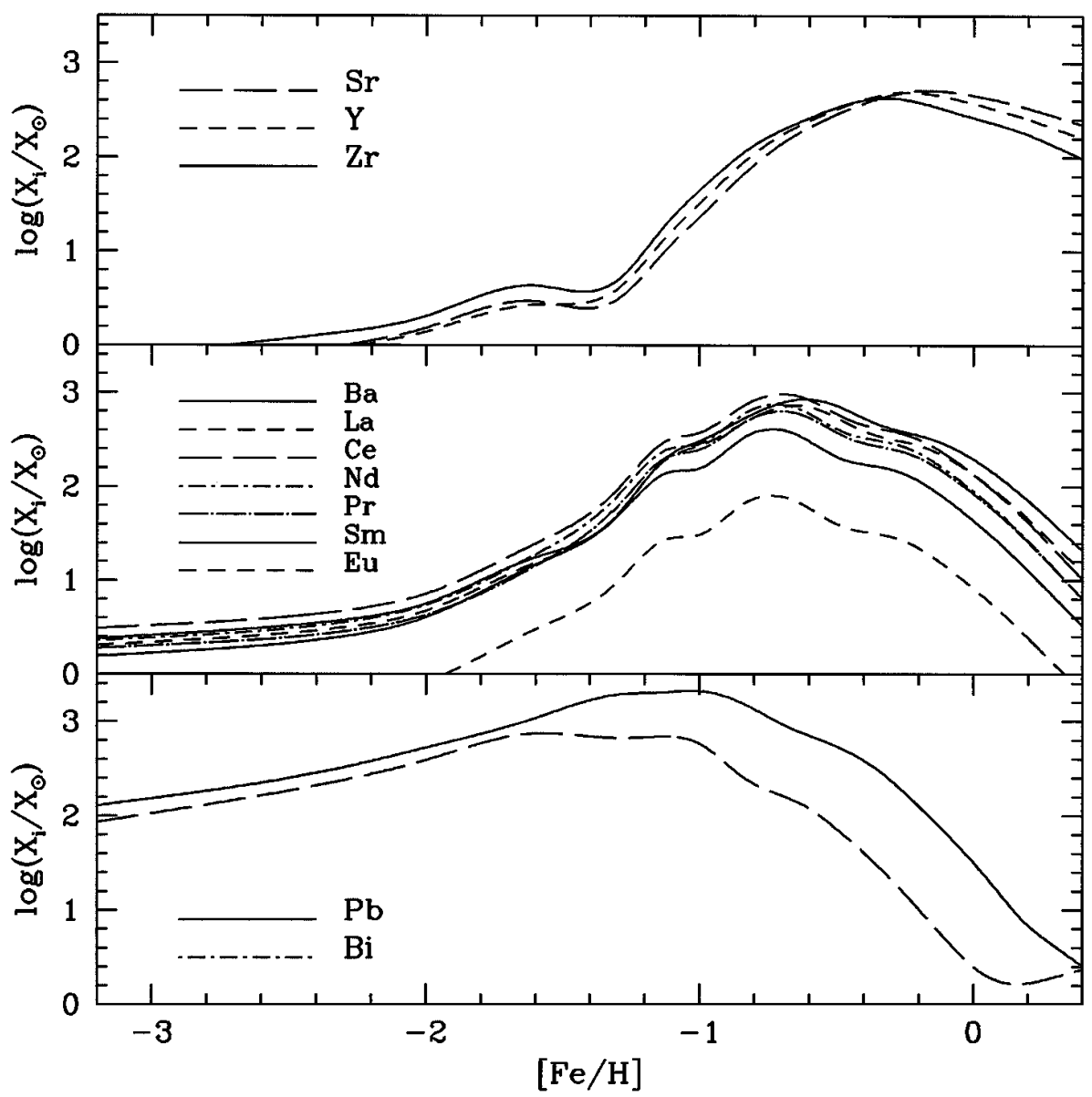

Figure 12 Logarithm of the enhancement factors relative to solar abundances as a function of metallicity for a $2-M_{\odot}$ model asymptotic giant branch star with the standard (ST) ${ }^{13} \mathrm{C}$ pocket discussed in the text. ${ }^{13} \mathrm{C}$ is burned under radiative conditions. It can be seen that $\mathrm{Pb}$ (dominantly ${ }^{208} \mathrm{~Pb}$ ) and $\mathrm{Bi}$ are the main slow neutron capture-process products in extremely metal-poor stars and that $\mathrm{Ba}$ and the rare-earth elements are enhanced for intermediate metallicities, whereas at near solar metallicity $\mathrm{Zr}, \mathrm{Y}$, and $\mathrm{Sr}$ are enhanced. This has clear implications for galactic chemical evolution. 
elements. Here the adopted stellar models are for $M=2 M_{\odot}$, but the trend shown has a general application, at least in the mass range $1.5 \leq M / M_{\odot} \leq 3-4$. One should notice that, in computing models for metal-poor stars, the initial abundance ratios are not simply a scaling of solar abundances but reflect the observed enhancement of $\alpha$-rich elements (e.g. O, Ne, Mg, etc) in population-II stars (e.g. see Gratton \& Ortolani 1986, Sneden et al 1991, Spite \& Spite 1991, Edvardsson et al 1993, Israelian et al 1998). In the calculations, the assumption was made that, below $[\mathrm{Fe} / \mathrm{H}]=-1$, oxygen is enhanced by 0.5 dex and other $\alpha$ elements are enhanced by 0.3 dex. For galactic-disk metallicities up to solar value, the enhancements are scaled linearly between these extreme values and zero. As is clear from Figure 12, at very low metallicity all of the $s$-element yields decrease for decreasing $Z$, reflecting the secondary nature of neutron captures that require seeds, mainly ${ }^{56} \mathrm{Fe}$, from the ISM. ${ }^{208} \mathrm{~Pb}$ (which reaches its maximum at $[\mathrm{Fe} / \mathrm{H}] \sim-1$ ) is the most abundantly produced nucleus in such metal-poor stars. This fact is sufficient to explain the galactic abundance of this isotope (Gallino et al 1998), without requiring any ad hoc strong component for the $s$ process (see also Section 3). At intermediate metallicities, $([\mathrm{Fe} / \mathrm{H}] \sim-0.8$, as typical of early galactic-disk stars), the Ba peak elements become the dominant products of neutron captures in AGB models. At still higher metallicities, the $\mathrm{Zr}$ peak elements become progressively more important. These nuclei are also efficiently contributed by more massive AGBs (IMS), which are instead never effective in producing much $\mathrm{Ba}$ (see the discussion of Section 4 and Busso et al 1988).

Some of the effects introduced by the primary nature of the ${ }^{13} \mathrm{C}$ neutron source on $s$-process abundances were recognized by Clayton (1988), who noticed that, although neutron captures are secondary in nature, a primary neutron source roughly implies that, for metallicities not far from solar, the neutron exposure behaves as $1 / Z$. The higher the neutron exposure $\tau$ is, the more the distribution of nuclei is peaked toward heavy species. Mathews et al (1992) also pointed out that an important role in controlling the dependence of the neutron flux on metallicity is played by the light neutron absorbers, especially by ${ }^{16} \mathrm{O}$. These workers simply scaled the solar main component distribution with $[\mathrm{Fe} / \mathrm{H}]$. However, not using suitable nucleosynthesis models, their analysis could not identify the strong non-linearity in the $s$-process trend versus metallicity (see Figure 12). In stellar models the trend of the neutron exposure as a function of $Z$ is actually more complex, and the dependence becomes $\sim Z^{-0.6}$ down to $Z=Z_{\odot} / 10$ (Gallino et al 1999).

For the same choice of the ${ }^{13} \mathrm{C}$ pocket, Figure 13 shows the distribution of the enhancement factors of $s$-only isotopes (and of isotopes mostly fed by the $s$ process) in the He intershell material cumulatively dredged up into the envelope, for metallicities $Z=(1 / 10,1 / 3,1 / 2,1) \times Z_{\odot}$. Here an initial mass $M=2 M_{\odot}$ was chosen, but the trend is generally applicable. Enhancements are computed for the initial composition; solar abundances are taken from Anders \& Grevesse (1989) and Grevesse et al (1996). As can be seen from the plot, at metallicities $Z=(1 / 3-1 / 2) \times Z_{\odot}$, the distribution for the $s$-only nuclei of $A \geq 85$ (heavy dots) is remarkably flat. This guarantees that such models are capable of mimicking the 


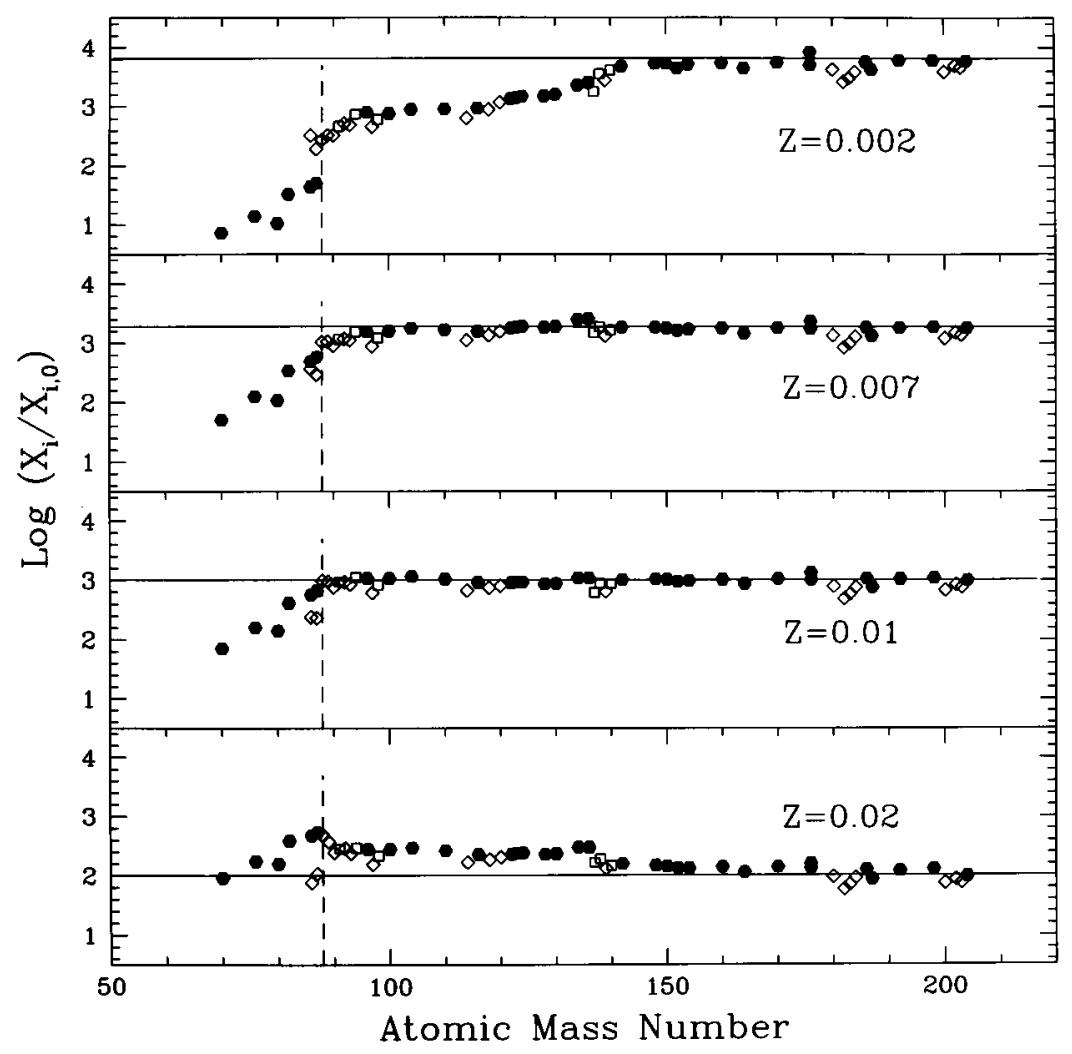

Figure 13 Distributions of enhancement factors of neutron-rich slow neutron capture $(s)$-process isotopes compared with the initial composition for different $Z$ values in the material cumulatively mixed to the surface of thermal-pulse-asymptotic giant branch stars. The horizontal line through $\mathrm{Bi}$ is drawn as a guide to the eye. Models are for a $2-M_{\odot}$ star, run at different metallicities, with the same standard $(S T)$ choice of the ${ }^{13} \mathrm{C}$ pocket. Heavy dots represent $s$-only nuclei; open squares are nuclei that are at least $80 \% s$ process; open diamonds are nuclei with $s$ contributions between $60 \%$ and $80 \%$. For decreasing $Z$, progressively heavier nuclei are favored. In the range of $Z=(1 / 2-1 / 3) \times Z_{\odot}$ the distribution is relatively flat, and $Z=0.01$ is a good approximation of the solar system main component. Note that the results are dependent on the ${ }^{13} \mathrm{C}$ pocket amount and inversely proportional to $Z$. For example, a case with $Z=0.01$ and ${ }^{13} \mathrm{C}$ pocket $1 / 2$ of the $\mathrm{ST}$ value would be indistinguishable from that of the bottom panel. 
solar main component; in particular the case at $Z=Z_{\odot} / 2$ reproduces it in remarkable detail, although, as previously stated, the main component is actually the result of the galactic average of many $s$-process events. Note that each distribution shown in Figure 13 can also be obtained in a different way, when both the metallicity and the amount of ${ }^{13} \mathrm{C}$ burnt are scaled by the same factor (upward or downward), so that the changes mutually compensate. For example, if one wishes to consider an increase of the ${ }^{13} \mathrm{C}$ pocket by a factor of 2 for $Z=0.02$, then the results will be very close to the curve shown for $Z=0.01$. It is through the interplay of the complex phenomena briefly outlined above that neutron captures in low-mass AGB stars ultimately produce $s$-process distributions that are suitable to account for the observational constraints at various metallicities, as we outline in the next subsections.

\subsection{Comparison with O bservations: Evolved Stars}

The above models and their dependence on metallicity allow a consistent interpretation of the abundances observed in AGB stars of various metallicities. A first constraint concerns the neutron density. High-resolution spectroscopic observations showed that AGB stars of the galactic disk that are $s$-process enhanced have a low $\mathrm{Rb} / \mathrm{Sr}$ abundance ratio. This ratio is sensitive to the neutron density and increases with it (Lambert et al 1995). Values of the $\mathrm{Rb} / \mathrm{Sr}$ ratio as low as those measured can be reproduced only if the neutron density is below $\sim 10^{7} \mathrm{n} \mathrm{cm}^{-3}$, as is indeed the case for radiative ${ }^{13} \mathrm{C}$ burning (Figure 14).

Important observational constraints derive from the abundances of elements near the major $s$-process peaks observed in AGB stars or their progeny and from their variation as a function of stellar metallicity. To explain such data, Busso \& Gallino (1997) constructed sequences of envelope compositions for AGB stars by following the mixing into the envelope of the $s$ elements produced in the intershell zone. The adopted models are the same as described above. Those authors compared their model abundances with spectroscopic observations of evolved stars. At galactic-disk metallicities (see Busso et al 1995b), the observed counterparts of the model stars are s-process- and carbon-enriched AGBs, both intrinsic and extrinsic, that is, both single objects of classes MS-S-C and giant or dwarf Ba stars. The Ba stars were most probably produced by the so-called wind accretion mass exchange in a binary system, by an AGB primary star that subsequently evolved to a white dwarf (e.g. see Jorissen \& Mayor 1992). Among metal-poor stars, $s$-process-enriched atmospheres are generally shown by binary $\mathrm{CH}$ and $\mathrm{Ba}$ stars, although an increasing role is played by newly available observations of post-AGB B-A-F supergiants (Decin et al 1998).

Comparisons with observations are shown by Figures 15 and 16. In particular, Figure $15 a$ and $b$ illustrates the degree to which the abundances of specific AGB stars can be reproduced. Here the observed compositions of a Ba star of the galactic disk (HR774) and of a $\mathrm{CH}$ subgiant of the galactic halo (CPD $\left.-62^{\circ} 6195\right)$ are modeled by mixing to the surface products of the He intershell, using stellar evolutionary computations of the appropriate metallicities $([\mathrm{Fe} / \mathrm{H}]=-0.3$ and 


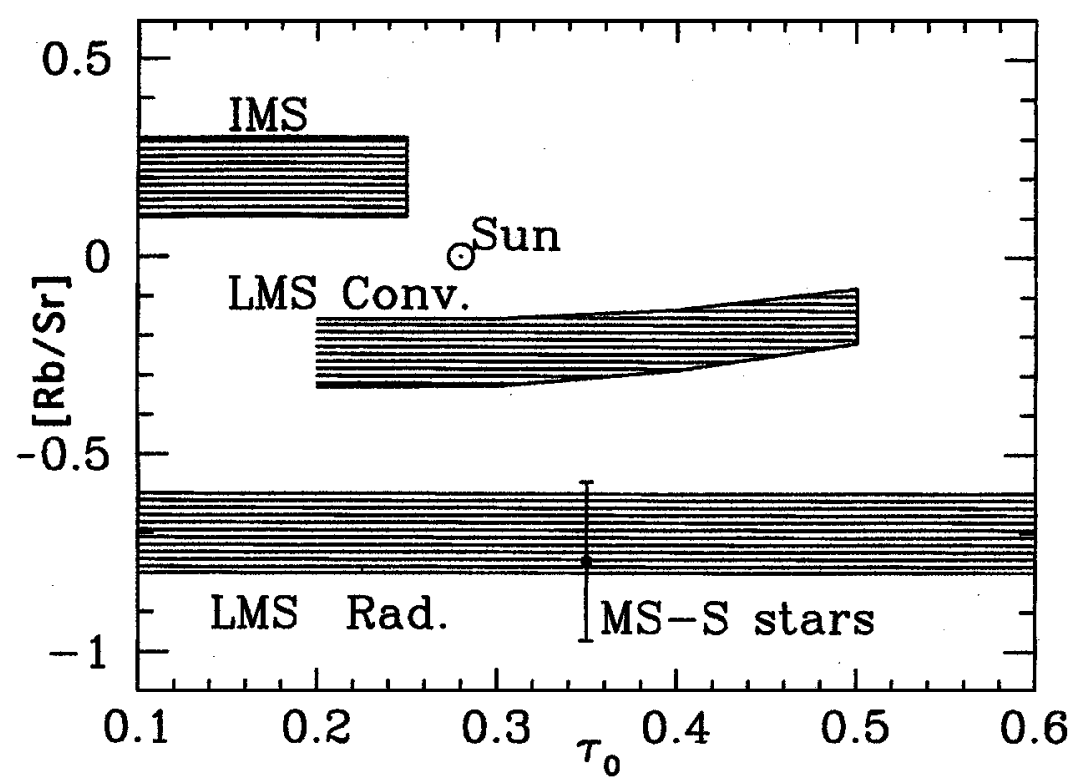

Figure 14 The average abundance ratio $\mathrm{Rb} / \mathrm{Sr}$, as deduced by Lambert et al (1995) from measurements in MS and S stars. The shaded regions cover the predictions we derive from $s$-process models in IMS and in LMS. In the first case, neutrons are produced by ${ }^{22} \mathrm{Ne}$ burning and in the second by ${ }^{13} \mathrm{C}$ burning. Convective (Conv.) and radiative $(\mathrm{Rad}.){ }^{13} \mathrm{C}$ burnings are shown, and it is clear that the convective model prediction is far above the observed value, whereas the radiative model is consistent with the data on MS stars.

-1 , respectively). A less detailed, but more comprehensive, comparison is shown in Figure 16, in terms of the [hs/ls] abundance ratios. This quantity (Luck \& Bond 1991) represents the mean logarithmic abundance ratio between the elements from $\mathrm{Ba}$ to $\mathrm{Sm}$ (hs) and those from $\mathrm{Sr}$ to $\mathrm{Zr}$ (ls), respectively. The abundances of various classes of observed AGB, post-AGB, or mass-transfer stars are indicated by different symbols. Model curves represent the computed surface compositions near the end of the AGB phase (typical dilution between envelope and He shell material 20) for some representative cases. They correspond to the ST choice of the ${ }^{13} \mathrm{C}$ pocket discussed above (heavy line) and to choices in which the ${ }^{13} \mathrm{C}$ content is scaled upward by a factor of 2.5 and downward by factors of 2 and 4 . The lowest curve represents the case of no ${ }^{13} \mathrm{C}$ pocket. The horizontal, dash-dotted line shows by comparison the maximum [hs/ls] attained by previous models, in which ${ }^{13} \mathrm{C}$ was supposed to burn in convective pulses, producing exponential distributions of neutron exposures (Gallino et al 1993, Busso et al 1995b). It is clear that the new models are compatible with several observations at low $Z$, especially for some $\mathrm{CH}$ giants, whereas the old convective burning models are not in accord with such observations (Vanture 1992). 

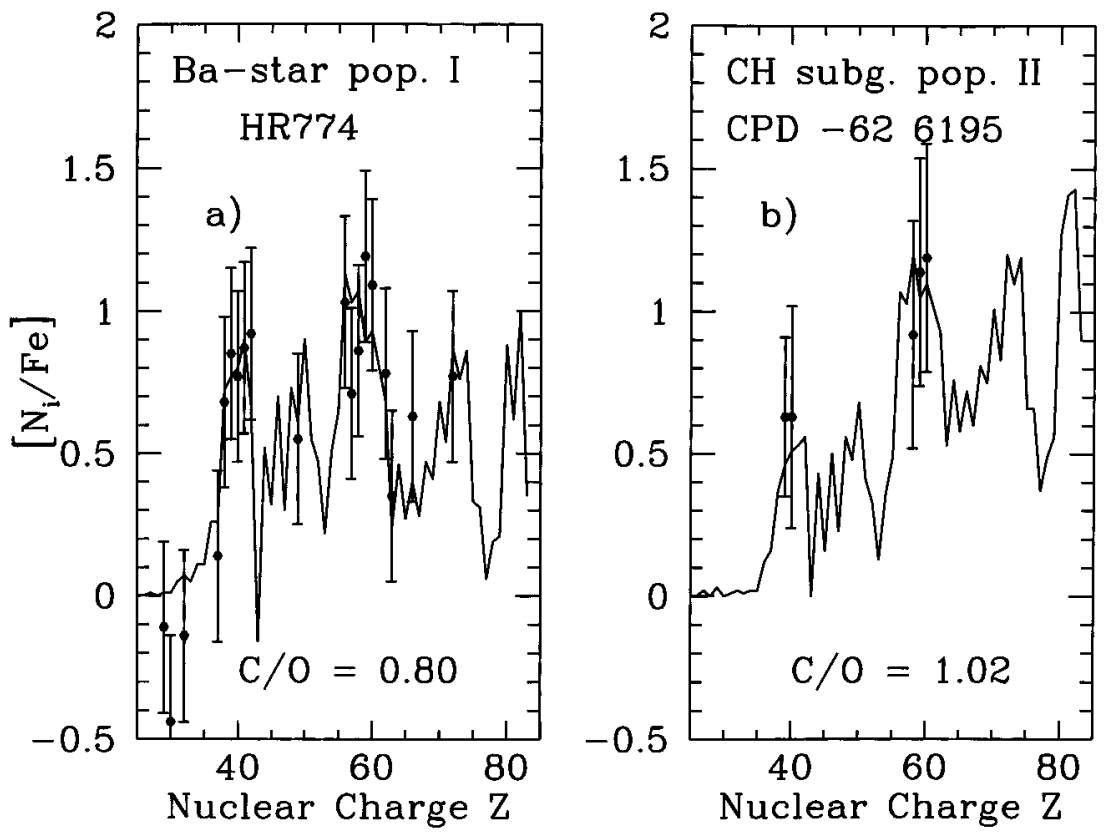

Figure 15 The slow neutron capture $(s)$-element abundances of $(a)$ a classical Ba star of the galactic disk $\left(Z=Z_{\odot} / 3\right)$ and $(b)$ a metal-poor $\mathrm{CH}$ subgiant $\left(Z=Z_{\odot} / 10\right)$. Observations are from (a) Smith (1984) and Tomkin \& Lambert (1983) and (b) Luck \& Bond (1991). Curves represent model surface compositions in models of the appropriate metallicity, using a Reimers' mass loss $(\eta=1.5)$, an initial mass $M=3 M_{\odot}$, and the standard (ST) choice of the ${ }^{13} \mathrm{C}$ pocket. Mixing to the envelope is performed during post-processing, using the TDU extensions found in a 3- $M_{\odot}$ model of $Z=Z_{\odot} / 3$ and stopping when the $\mathrm{C} / \mathrm{O}$ ratio is matched. As is seen from the figures, this procedure automatically produces a good fit to the $s$-process abundances, without adjusting other parameters.

\subsection{Comparison with O bservations: Unevolved Stars}

It has been known for almost two decades that elements dominantly produced by the $s$ process, like $\mathrm{Sr}, \mathrm{Ba}$, or La, apparently behave like primary products in field dwarfs or first-ascent red giants of population $\mathrm{I}$. This means that $\left[\mathrm{X}_{i} / \mathrm{Fe}\right]$ remains roughly constant as a function of $[\mathrm{Fe} / \mathrm{H}]$, within the (large) observational uncertainties. The growing sets of data on metal-poor stars then showed that those abundance ratios remain consistently high even at lower metallicities, for stars belonging to the galactic halo (e.g. see Ryan et al 1991, Gratton \& Sneden 1994, McWilliam et al 1995, McWilliam 1995, 1998). The interpretation of such a behavior is not trivial and involves consideration not only of the $s$-process, but also of the $r$-process contribution to each nuclide. 


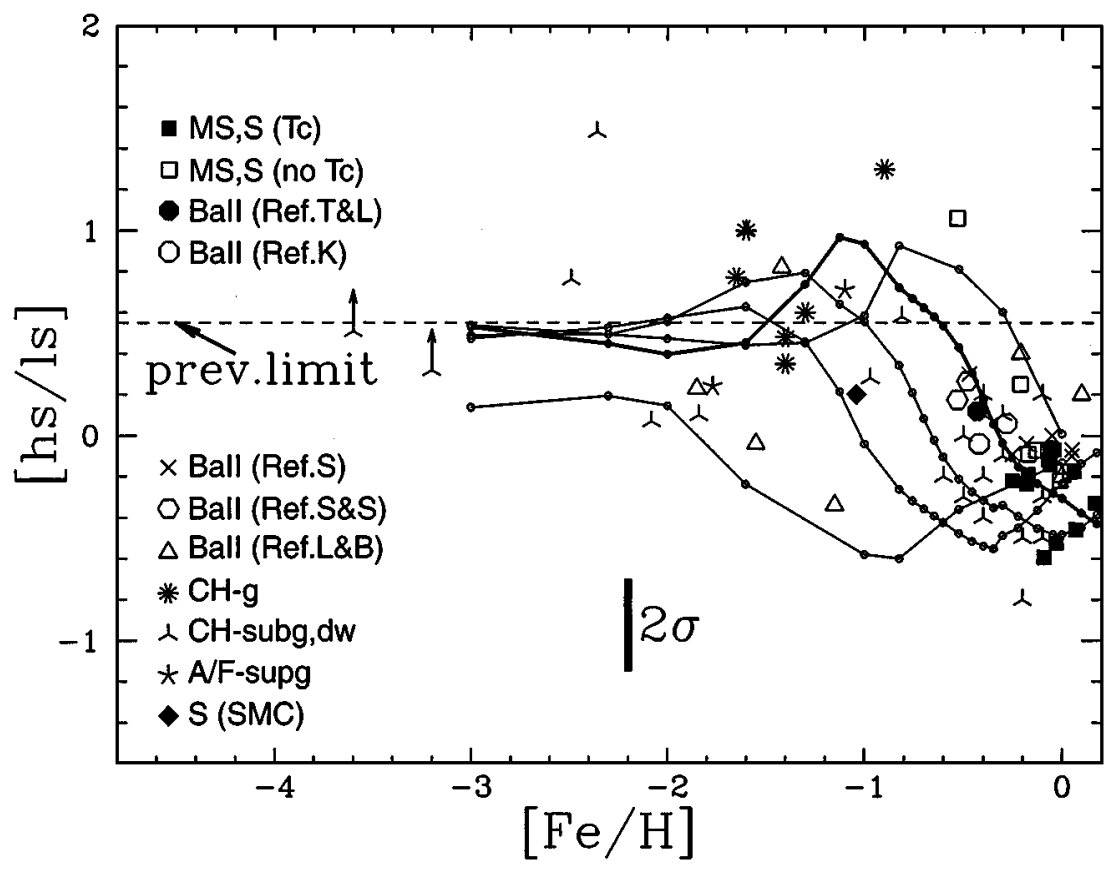

Figure 16 The logarithmic ratio [hs/ls] between heavy ( $\mathrm{Ba}, \mathrm{La}, \mathrm{Nd}, \mathrm{Sm})$ and light slow neutron capture elements $(\mathrm{Y}, \mathrm{Zr})$ as a function of $[\mathrm{Fe} / \mathrm{H}]$. Observations are from Smith \& Lambert (1990), Luck \& Bond (1981, 1984, 1985, 1991), Vanture (1992), Plez et al (1993), Luck et al (1990), Waelkens et al (1991), and Sneden \& Parthasarathy (1983). Each model curve represents surface compositions obtained with a particular choice for the ${ }^{13} \mathrm{C}$ pocket and various initial metallicities. The curve in bold is for the standard (ST) case. Other curves are for choices of the ${ }^{13} \mathrm{C}$ pocket with extensions scaled by $0.25,0.5$, and 2.5 compared with the ST case. The lowest curve is for no ${ }^{13} \mathrm{C}$ pocket. The dispersion in the observations may indicate a real spread in the magnitude of the ${ }^{13} \mathrm{C}$ pocket. These models appear to account for the observations of $\mathrm{CH}$ giants. The horizontal dashed line shows the maximum [hs/ls] with previous (convective) ${ }^{13} \mathrm{C}$-burning models with an exponential distribution of neutron exposures.

We discuss the above trend by using Ba as an example (Figure 17a,b), following results obtained by Travaglio et al (1998). These workers used the stellar yields shown in Figures 12 and 13 and computed the chemical enrichment of the galaxy in neutron-rich elements, adopting a previously established chemical-evolution model (Ferrini et al 1992) that explained the galactic evolution of major light elements. Models that consider only the $s$-process contribution to $\mathrm{Ba}$ as coming from the integrated production of AGB stars throughout the galactic life yield the continuous line shown in Figure 17a. A comparison with observations of unevolved stars shows a sharp disagreement for low metallicities. However, for stars of the galactic disk, the model curve roughly reproduces the observed data. 


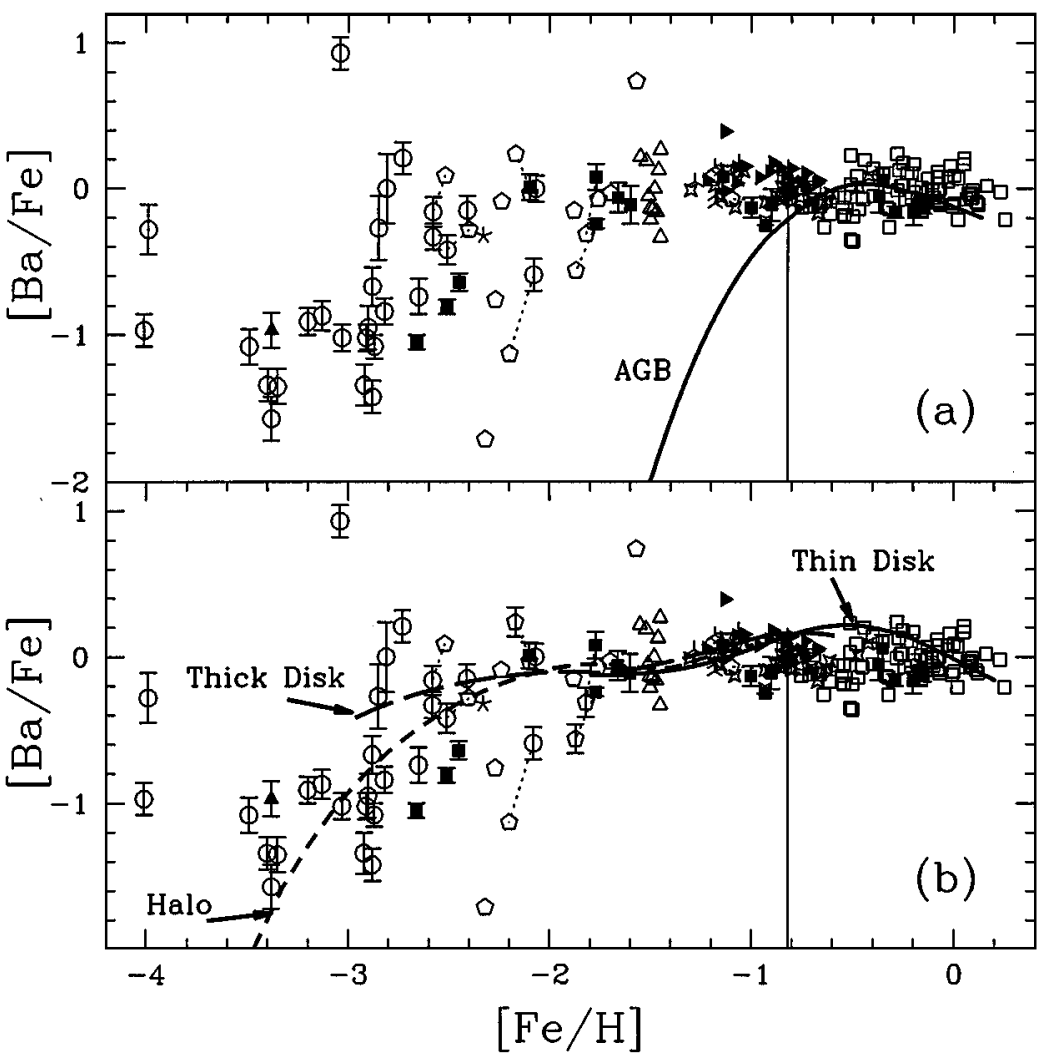

Figure 17 Observed abundances of $[\mathrm{Ba} / \mathrm{Fe}]$ in unevolved stars as a function of $[\mathrm{Fe} / \mathrm{H}]$. $a$. The curve for galactic enrichment of purely slow neutron capture $(s)$-process $\mathrm{Ba}$, as produced by asymptotic giant branch stars of different metallicities calculated by using the galactic chemical-evolution model of Travaglio et al (1998). The curve shows a clear disagreement with the data for low-metallicity stars but provides a reasonable reproduction of $\mathrm{Ba}$ abundances in the galactic disk. The $s$ contribution to Ba dominates after the first Gyr of galactic evolution (corresponding to the vertical line in the two panels). $b$. Results after adding the $r$-process contribution, determined to be $20 \%$ of the solar values. Different model curves in panel $b$ represent the halo population (short dashed line), thick disk (long dashed line), and thin disk of the galaxy (continuous line). In panel $a$ these curves are indistinguishable (see Travaglio et al 1998, for details).

Here the lower and upper limits of AGB stellar masses were taken to be 2 and 4 $M_{\odot}$, respectively, and the ST choice for the ${ }^{13} \mathrm{C}$ pocket was made. The results were found to remain qualitatively the same when the upper mass limit of AGB stars is increased, because more massive AGBs do not contribute much to Ba. Similarly, the curves are about the same when a reasonable spread in $s$-process efficiencies at each metallicity is taken into account. The $s$-process fraction of $\mathrm{Ba}$ at $t=t_{\odot}$ is calculated to be $80 \%$. Travaglio et al (1999) estimated the $r$ contribution to Ba 
by subtracting the $s$ contribution shown in Figure $17 a$ : for the sun, at $Z=Z_{\odot}$, $\left(1-N_{s} / N\right)_{\mathrm{Ba}}=0.20$.

From Figure 17a, one can see that the $s$-process contribution begins to dominate the galactic enrichment for $[\mathrm{Fe} / \mathrm{H}] \simeq-1$, corresponding to a galactic age of $\sim 1 \mathrm{Gyr}$. At lower $[\mathrm{Fe} / \mathrm{H}]$ values, the $s$ process is clearly incapable of explaining the observed data. This is linked both to the long time scales of LMS evolution and to the efficiency of $s$-element production in AGB stars of different metallicities, as summarized in Figures 12 and 13. Indeed, at low $Z$ values the Ba peak nuclei do not receive strong contributions from AGB stars, and it is conceivable that they are dominated by their $r$-process components, as early suggested by Truran (1981). It is in fact known from observations that the heavy $r$-process nuclei are produced by short-lived massive stars, presumably in supernova explosions that also produce ${ }^{16} \mathrm{O}$ (cf Cowan et al 1996).

Travaglio et al (1999) made the hypothesis that the $r$-process yield of Ba comes from moderately massive supernovae of type II $\left(8-10 M_{\odot}\right)$. Hence, they assumed that the yield of $\mathrm{Ba}$ relative to $\mathrm{O}$ is independent of metallicity for the producing supernovae. This means that, at low $Z$, where the $\mathrm{O} / \mathrm{Fe}$ ratio is higher than in the sun (by a factor of 3-4), the $\mathrm{Ba}_{r} / \mathrm{O}$ ratio increases by the same factor. In the above hypotheses, Travaglio et al (1999) reconstructed the galactic history of Ba, including both $r$ and $s$ contributions. The results are shown in Figure 17b. Here the model curves for the three major components of the galaxy are shown (halo, short dashed line; thick disk, long dashed line; thin disk, continuous line). The curves extend through the intervals of metallicity (in part mutually overlapping) over which star formation is active in the three zones. As can be seen from the plot, the model curves are compatible with the bulk of observed data. The galactic evolution model used for the halo phase implies a well-homogeneized situation and cannot explain the large scatter of spectroscopic data. This spread has been commented upon by various authors (Griffin et al 1982; Gilroy et al 1988, Ryan et al 1991; McWilliam et al 1995, McWilliam 1998). As more and more data is collected, the scatter appears to be largely intrinsic in nature, a clear indication of an inhomogeneous evolution of the halo, with incomplete mixing of stellar ejecta over the extended interstellar medium. This role can now be studied using numerical simulation techniques, either as a stochastic analysis with Monte Carlo simulation (see McWilliam \& Searle 1998) or with $N$-body smooth particle hydrodynamics (SPH) (Raiteri et al 1999) to create models of the chemical and dynamical evolution of the Galaxy to account for the incomplete mixing. A similar effort has been made by Ishimaru \& Wanajo (1999). There is a wide scatter that is not easily explained. Raiteri et al (1999) show that part of the scatter at low metallicity can be caused by observations of stars from different galactic subsystems. Later, when star formation becomes limited to the thin disk, the $s$-process component to $\mathrm{Ba}$ from AGB stars becomes dominant. These results are in support of the suggestions by Truran (1981) that at low $Z$ the abundance of neutron-rich nuclei is caused by the $r$ process. Important progress in this respect has been obtained through observations in very metal-poor stars (Gilroy et al 1988; Sneden et al 1996, 1998; Norris et al 
1997). What is relevant in the present context is that the $r$ component to Ba was deduced as a difference, after the $s$ contribution from AGB stars was modeled.

From the examples discussed above we can say that the present status of $s$-process nucleosynthesis in AGB stars appears to offer an understanding of the behavior of n-capture nuclei in stars of the galactic disk. It also serves to infer the expected $r$-process contributions and hence to aid in a reconstruction of the whole galactic history of the nucleosynthesis of neutron-rich isotopes.

\section{SOURCESOF SHORT-LIVED NUCLEI IN THE EARLY SOLAR SYSTEM}

From studies of meteorites that formed early in solar system history, which were based on long-lived (mean life $\bar{\tau} \geq 10^{9}$ years) radioactive parents and their daughter products, it has been established that the age of the solar system is about $4.556 \times 10^{9}$ years (cf Bahcall et al 1995). The problem of the galactic time scale and the abundances of the actinides was formulated by Burbidge et al (1957) and Fowler \& Hoyle (1960). In addition to the long-lived actinides, many of the meteorites also contain evidence that some radioactive nuclei with relatively short lifetimes were present at the time of their formation. The extent to which a radioactive nucleus will survive until the present time is dependent on its mean lifetime. The shortest-lived species that is preserved today from the beginning of the solar system, with a small but significant abundance, is ${ }^{235} \mathrm{U}\left(\bar{\tau}=1.015 \times 10^{9}\right.$ years $)$. For an isotope with $\bar{\tau}=2 \times 10^{8}$ years, the initial abundance would be decreased today by a factor of $10^{10}$. We thus consider the current inventory of all original radioactivities in the solar system with $\bar{\tau} \leq 1 \times 10^{8}$ years to be extinct (this does not relate to those few nuclei produced later by cosmic rays or local nuclear interactions or swept up from the interstellar medium). Considering long-term production in the history of the galaxy (over a time $T$ ) prior to solar system formation, the abundance of a stable nuclide (S) is $N_{S}(T)=P_{S}\langle p\rangle T$. Here $P_{S}\langle p\rangle$ is the average production rate, assumed to be the product of a time-invariant stellar production factor $P_{S}$ and of a time-dependent scaling factor $p(t)$. A short-lived radioactive nuclide $\mathrm{R}$ produced in the same process that is in steady state in the ISM between production and decay will have the abundance $N_{R}(T) \approx P_{R} p(T) \bar{\tau}_{R}$. Here $p(T)$ is the value of the scaling factor close to the formation of the solar system. Then $N_{R}(T) / N_{S}(T) \approx P_{R} p(T) \bar{\tau}_{R} / P_{S}\langle p\rangle T$. If material is separated from the ISM for a period $\Delta$ without further addition of freshly nucleosynthesized nuclei, then its abundance will be decreased by $\exp \left(-\Delta / \bar{\tau}_{R}\right)$. For nuclides with $\bar{\tau} \leq 10^{6}$ years (e.g. ${ }^{41} \mathrm{Ca},{ }^{26} \mathrm{Al}$ ), isolation times of a few Myr are sufficent to greatly diminish their abundance (see Schramm \& Wasserburg 1970 for a full treatment).

Consider a reference state in which solar materials first began to form from the interstellar medium, during which a diverse set of radioactive nuclei was present. Their existence in the early solar system cannot be determined directly by measuring them today but depends on showing that their decay products are present and 
that these decay products are quantitatively correlated with the chemical properties of the parent element, not with the daughter element. For a sample that has been preserved as an isolated system from its initial state (0) until today, the relationship governing an extinct radioactive parent nucleus $\mathrm{R}$ that decays to a daughter D (a different element) is $N_{D}{ }^{\text {today }}=N_{D}{ }^{0}+N_{R}{ }^{0}$. Here $N_{D}{ }^{0}$ is the initial number of D nuclei. Consider now a stable isotope I (index) of the same element as the daughter and another stable isotope $\mathrm{S}$ (stable) of the same element as the unstable parent $\mathrm{R}$. The isotope $\mathrm{S}$ serves as a surrogate of $\mathrm{R}$ of the same element. One has $\left(N_{D} / N_{I}\right)^{\text {today }}=\left(N_{D} / N_{I}\right)^{0}+\left(N_{R} / N_{S}\right)^{0}\left(N_{S} / N_{I}\right)^{\text {today }}$. Here $N_{I}^{\text {today }}=N_{I}^{0}$ and $N_{S}{ }^{\text {today }}=N_{S}{ }^{0} \cdot N_{D}{ }^{0} / N_{I}^{0}$ is the initial isotopic ratio before decay of the parent nucleus $\mathrm{R}$. The above also applies to the bulk solar ratio today, using solar abundance ratios. Thus for ${ }^{26} \mathrm{Al}$ (which decays to ${ }^{26} \mathrm{Mg}$ ) we use the index isotope ${ }^{24} \mathrm{Mg}$, and for ${ }^{107} \mathrm{Pd}$ (which decays to ${ }^{107} \mathrm{Ag}$ ) we use the index isotope ${ }^{109} \mathrm{Ag}$. In these examples, the ratio $N_{S}{ }^{\text {today }} / N_{I}{ }^{\text {today }}$ corresponds to ${ }^{27} \mathrm{Al} /{ }^{24} \mathrm{Mg}$ and to ${ }^{108} \mathrm{Pd} /{ }^{109} \mathrm{Ag}$, respectively, whereas $\left(N_{R} / N_{S}\right)^{0}$ would correspond to $\left({ }^{26} \mathrm{Al} /{ }^{27} \mathrm{Al}\right){ }^{0}$ or $\left({ }^{107} \mathrm{Pd} /{ }^{108} \mathrm{Pd}\right)^{0}$. This exhibits the relationship that the isotope ratio $N_{D}{ }^{\text {today }} / N_{I}{ }^{\text {today }}$ linearly correlates with the ratios of $N_{S}{ }^{\text {today }} / N_{I}{ }^{\text {today }}$. A sample with none of the parent element (neither R nor $\mathrm{S}$ ) would show the initial value for the ratio of the daughter nuclide to I. To see observable effects in $N_{D} / N_{I}$ today, the samples studied must have been formed early with a large range in values of $N_{S} / N_{I}$ and must have been preserved until today as isolated systems. Evidence for the presence of the radioactive nuclides can thus be found only by isotopic shifts relative to the solar value, which requires that there have been very large chemical fractionates of the element represented by $\mathrm{S}$ as compared with the element represented by I at the time of formation (Figure 18). By chemical fractionation we mean that a phase (crystal or liquid) is formed from some uniform bulk material but has major differences in proportions of some elements (large variations in $N_{S} / N_{I}$ for a sample) as compared with the bulk material (e.g. the crystallization of a salt from a liquid or the condensation of $\mathrm{Al}_{2} \mathrm{O}_{3}$ or $\mathrm{SiC}$ crystals from a gas of stellar composition). For simplicity we assume that the solar nebula was once reasonably well mixed. For a complete presentation of the formalism, see Lee et al (1977) and Wasserburg (1987).

There is now clear evidence for the presence of many radioactive nuclei in the ESS (see Table 1 and Figure 18). The first short-lived nuclide discovered was ${ }^{129}$ I by JH Reynolds (1960). It was a crucial discovery. The discovery of ${ }^{26} \mathrm{Al}$ in the early solar system (Lee et al 1976, 1977) juxtaposed the presence of two radioactive nuclides $\left({ }^{129} \mathrm{I}\right.$ and $\left.{ }^{26} \mathrm{Al}\right)$ with grossly disparate lifetimes. It was to take over three decades to obtain a fuller assessment of the early solar system inventory. We have indicated our assessment of the degree of certainty with which the presence of these nuclei has been established and their abundance relative to a stable or longlived isotope of the same element $\left(N_{R} / N_{S}\right)$ in the ESS at $4.55 \times 10^{9}$ years ago. References cited here are usually for recent publications covering the observations. A recent review of the evidence and interpretation of the short-lived nuclei is given by Podosek \& Nichols (1997). More extensive literature references can be found in these citations. We indicate the nucleosynthetic processes associated with these nuclei. An extensive summary of the ${ }^{26} \mathrm{Al}$ as observed in the galaxy from gamma 
spectroscopy is given by Prantzos \& Diehl (1996). Abundances of $r$-process nuclei in the ISM have been calculated for constant production rates derived by $r$-process systematics, assuming a timescale $T=10^{10}$ years before isolation of the solar system. For ${ }^{60} \mathrm{Fe},{ }^{53} \mathrm{Mn},{ }^{41} \mathrm{Ca}$, and ${ }^{26} \mathrm{Al}$, we have used estimates of supernova type-II (SNII) yields from Woosley \& Weaver (1995) and an SNII rate of 0.03 SN year $^{-1}$ for the galaxy (see also Wasserburg et al 1996, 1998). Table 1 makes the implicit assumption of a unique and time-invariant $r$-process site, associated with $\mathrm{SN}$. Both $r$ and $p$ processes are currently believed to be associated with supernovae (Cowan et al 1996). The simple case for uniform production (hereafter UP) made in Table 1 is an approximation representing a stochastic input of discrete events. If we consider a model with discrete events, then there is a distinct granularity in the abundances caused by the most recent sources. A late-stage addition close to the time of formation of the solar system will enhance the short-lived nuclei. Note that the isotopic ratios inferred from meteoritic studies for the various species are from a multiplicity of objects found in meteorites or from different bulk meteorites that formed under different conditions at different times. This is particularly important for nuclei with $\bar{\tau}<10^{7}$ years, (i.e. ${ }^{41} \mathrm{Ca},{ }^{26} \mathrm{Al},{ }^{60} \mathrm{Fe}$, and ${ }^{53} \mathrm{Mn}$ ). Their abundance critically depends both on the initial inventory at some reference time $(0)$ near the formation of the solar system and the time of formation of the meteorite or meteorite subsystem that was studied. These times are not, in general, known (Figure 19). Aspects of the theoretical and observational estimates of various short-lived nuclei and the accretionary lifetime of the solar nebula are given by Podosek \& Cassen (1994). It is evident from studies of meterorites that these objects contain a reliable record of the first several million years of solar system history and also of individual stellar contributions to the early sun (cf Anders \& Zinner 1993).

If we consider the abundances of these nuclei at the time of formation of the solar system, we must recognize that they came from various stellar sources. It is actually well established that some of the materials in the ISM from which the solar system formed are assemblages of debris from different stars born in different molecular clouds at different times (Figure 20). For radioactive nuclei with mean lives sufficiently long compared with the local astration rate, the abundance will reflect the longer-term galactic inventory in the ISM. In contrast, for those radioactive nuclei with short mean lives, it may be required that their abundance reflects injection of freshly synthesized material from stellar sources immediately before the solar nebula formed (Figure 20). The earliest objects formed within the solar system by melting, cooling, and crystallization are considered to be the calcium-aluminum-rich inclusions (CAIs). In contrast, the chondrites are assemblages of early and later formed solar system debris and usually contain in their matrix a very small amount of unprocessed presolar grains (cf Huss \& Lewis 1995). They thus contain a variety of different objects (e.g. CAIs, chondrules, matrix, and metal) formed at different times and each with its own previous history. The other objects (e.g. iron meteorites and eucrites) are the result of planetary melting that took place after the formation of CAIs and, presumably (but not certainly), after chondrites were assembled. Most meteorites have been subjected to some heating and chemical changes after their "initial" formation (metamorphism). We note 


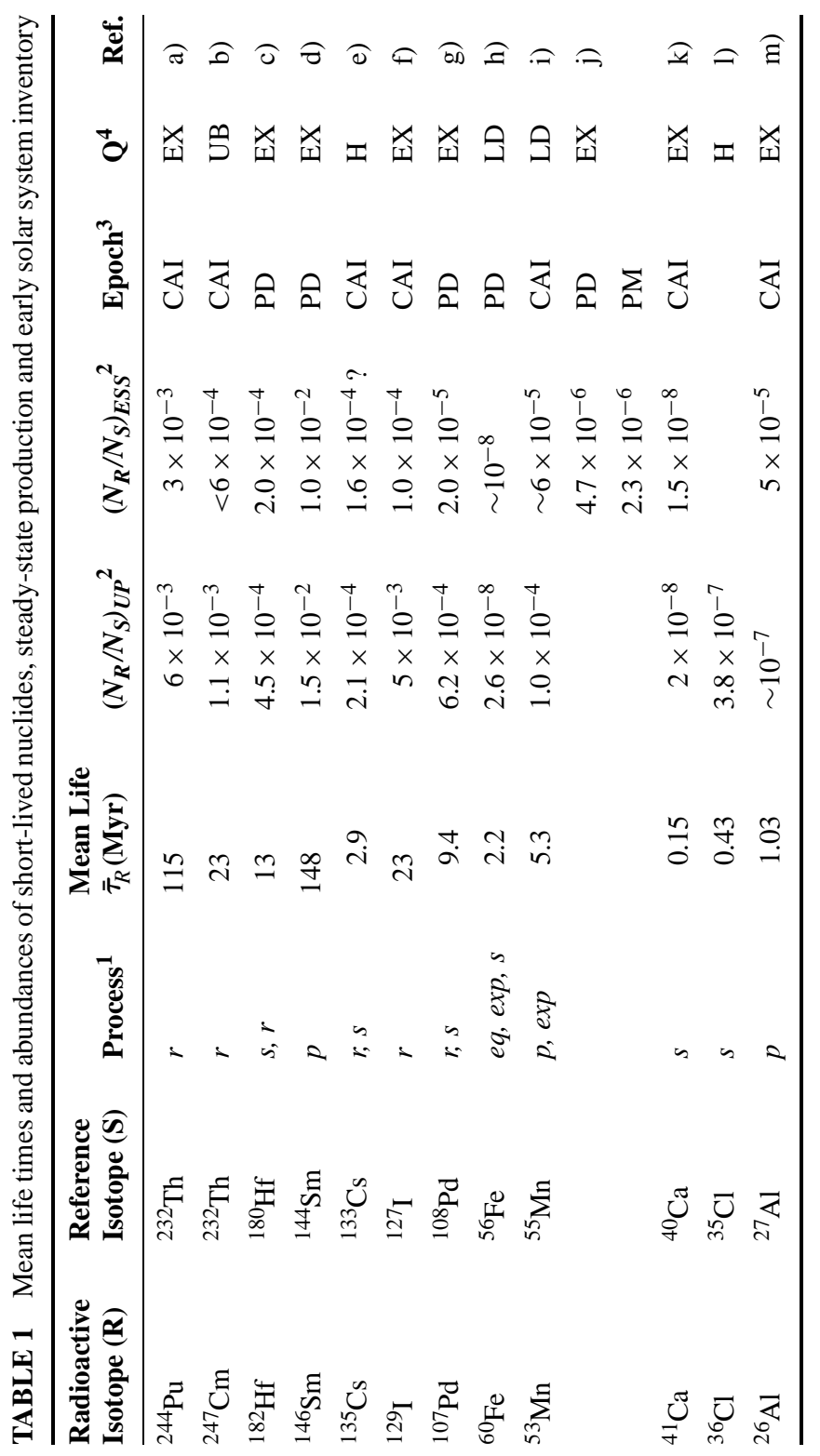




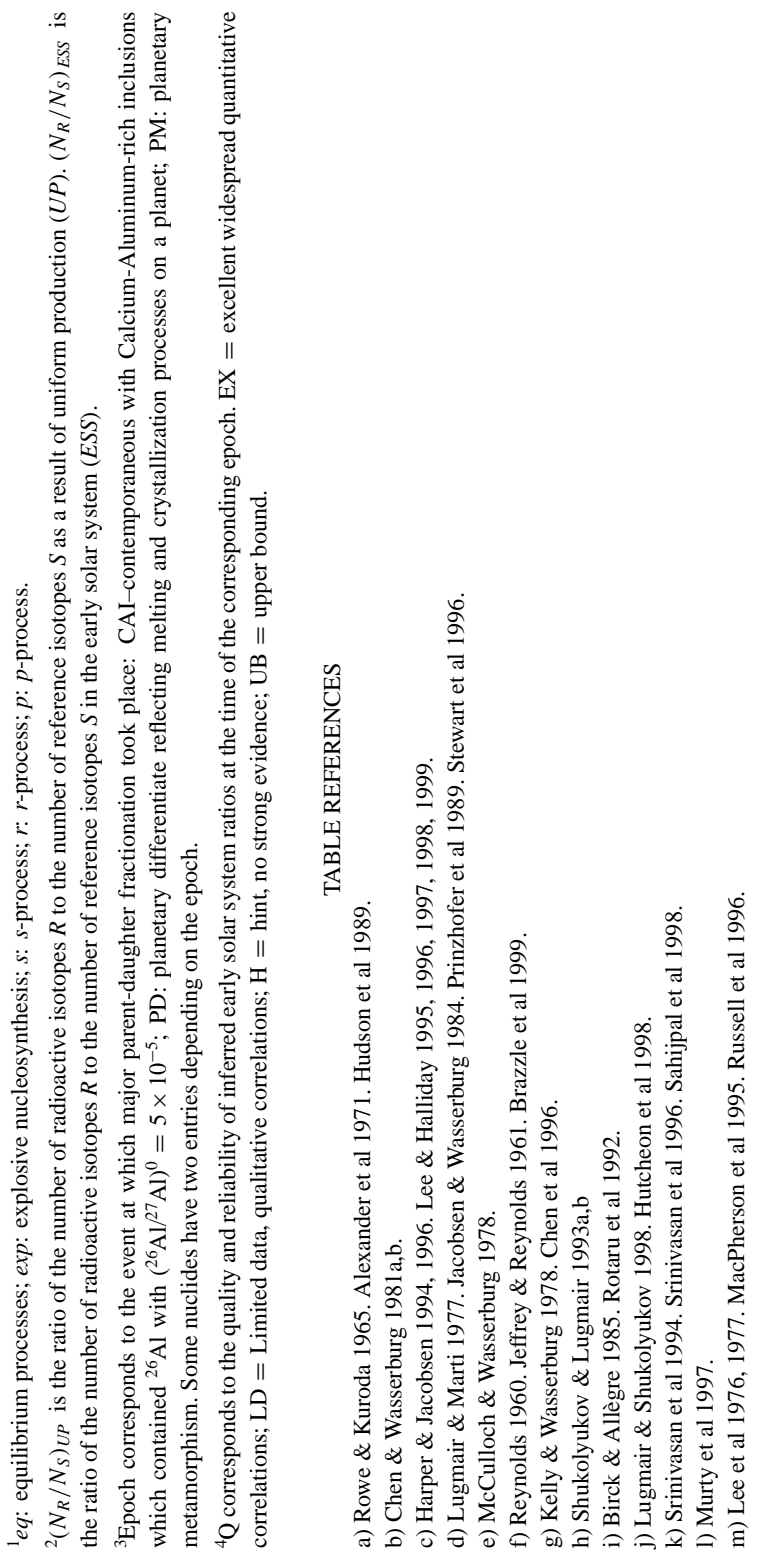



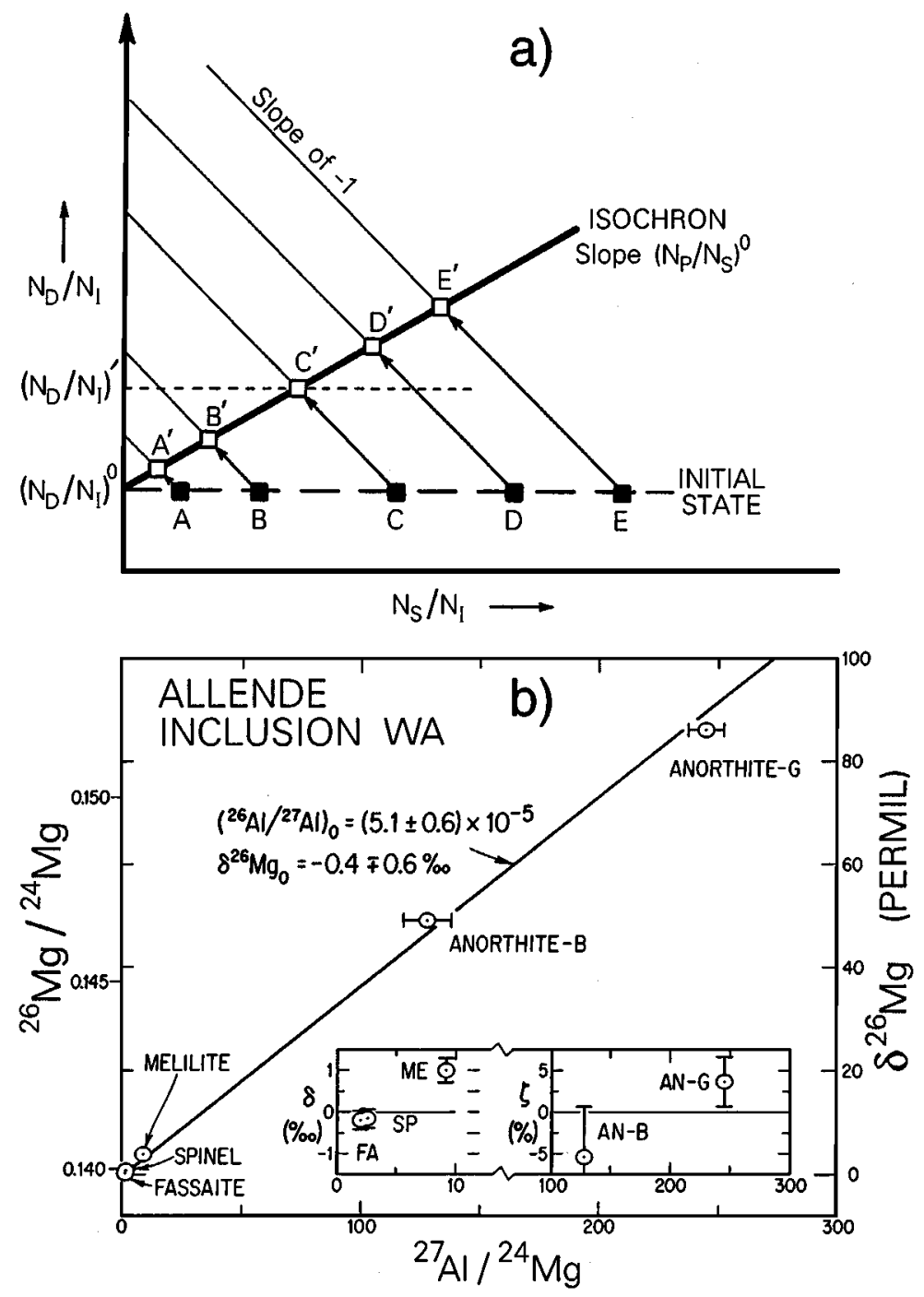

Figure $18(\boldsymbol{a}, \boldsymbol{b}) \quad a$. Evolution diagram of $N_{D} / N_{I}$ for a series of samples with different values of $N_{S} / N_{I}$ but with the same initial value $\left(N_{D} / N_{I}\right)^{0}$. The evolution through time for sample $\mathrm{E}$ follows the line of slope -1 to the point $E^{\prime}$ when the parent nucleus $\mathrm{P}$ has completely decayed; all such samples will lie on the isochron with slope $\left(N_{P} / N_{S}\right)^{0}$ (after Wasserburg 1987); $b$. Plot of ${ }^{26} \mathrm{Mg} /{ }^{24} \mathrm{Mg}$ vs ${ }^{27} \mathrm{Al} /{ }^{24} \mathrm{Mg}$, showing the correlation of excess ${ }^{26} \mathrm{Mg}$ with ${ }^{27} \mathrm{Al}$. Data are from various phases with different ${ }^{27} \mathrm{Al} /{ }^{24} \mathrm{Mg}$ from the same calcium-aluminum-rich inclusion from the Allende meteorite. This demonstrates the presence of ${ }^{26} \mathrm{Al}$ at an abundance of ${ }^{26} \mathrm{Al} /{ }^{27} \mathrm{Al}=5 \times 10^{-5}$ at the time of crystallization. Inset shows the deviations of the data from the line in parts per thousand (Lee et al 1977). 

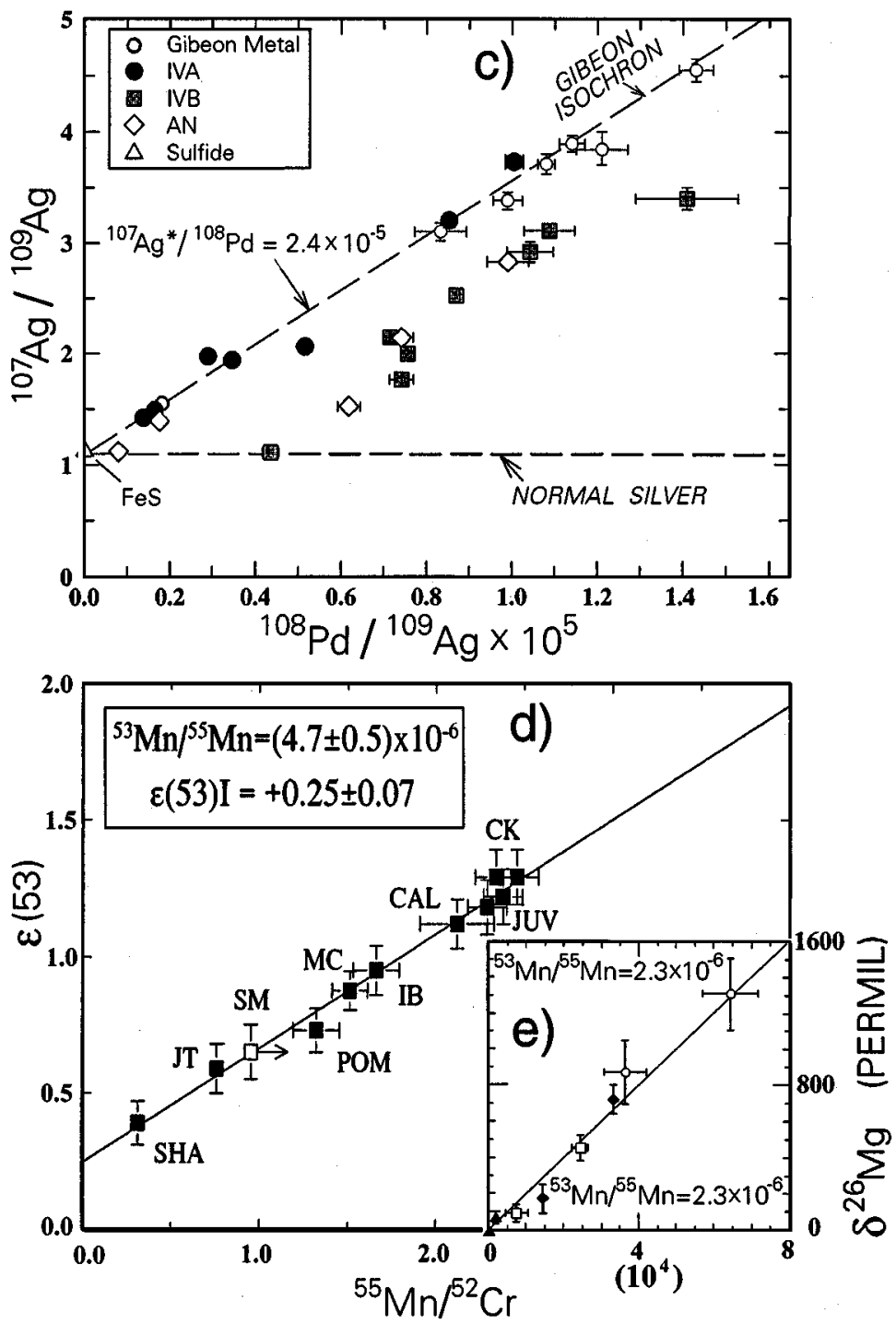

Figure $18(\boldsymbol{c}-\boldsymbol{e}) \quad c$. Correlation of ${ }^{107} \mathrm{Ag} /{ }^{109} \mathrm{Ag}$ with ${ }^{108} \mathrm{Pd} /{ }^{109} \mathrm{Pd}$ in different iron meteorites, demonstrating the presence of ${ }^{107} \mathrm{Pd}$ in these planetary segregates at the time they formed. A suite of different samples from the meteorite Gibeon defines the reference isochron (after Chen \& Wasserburg 1996). Some iron meteorites appear contemporaneous with Gibeon, and others appear more recent. $d$. Correlation of ${ }^{53} \mathrm{Cr} /{ }^{52} \mathrm{Cr}$ vs ${ }^{55} \mathrm{Mn} /{ }^{52} \mathrm{Cr}$, showing the presence of ${ }^{53} \mathrm{Mn}$ in different eucritic meteorites (basaltic rocks) at the time the melts formed on their parent planet (Lugmair \& Shukolyukov 1998). Here $\epsilon_{53} \equiv\left[\left({ }^{53} \mathrm{Cr} /{ }^{54} \mathrm{Cr}\right)_{\text {sample }} /\left({ }^{53} \mathrm{Cr} /{ }^{54} \mathrm{Cr}\right)_{\odot}-1\right] \times 10^{4} . e$. Isochron for chondrules from a $\mathrm{CV}$ meteorite (same general class as Allende) also demonstrating the presence of ${ }^{53} \mathrm{Mn}$ but showing that large Fe and very large Mn enrichments caused by planetary metamorphism in the olivine, occurring $5 \times 10^{6}$ years after the eucrites were crystallized (Hutcheon et al 1998). This illustrates some of the basic problems of establishing a truly refined, self-consistent chronology. 


\section{$\mathcal{A}$}

CONG-TERM PRESOLAR

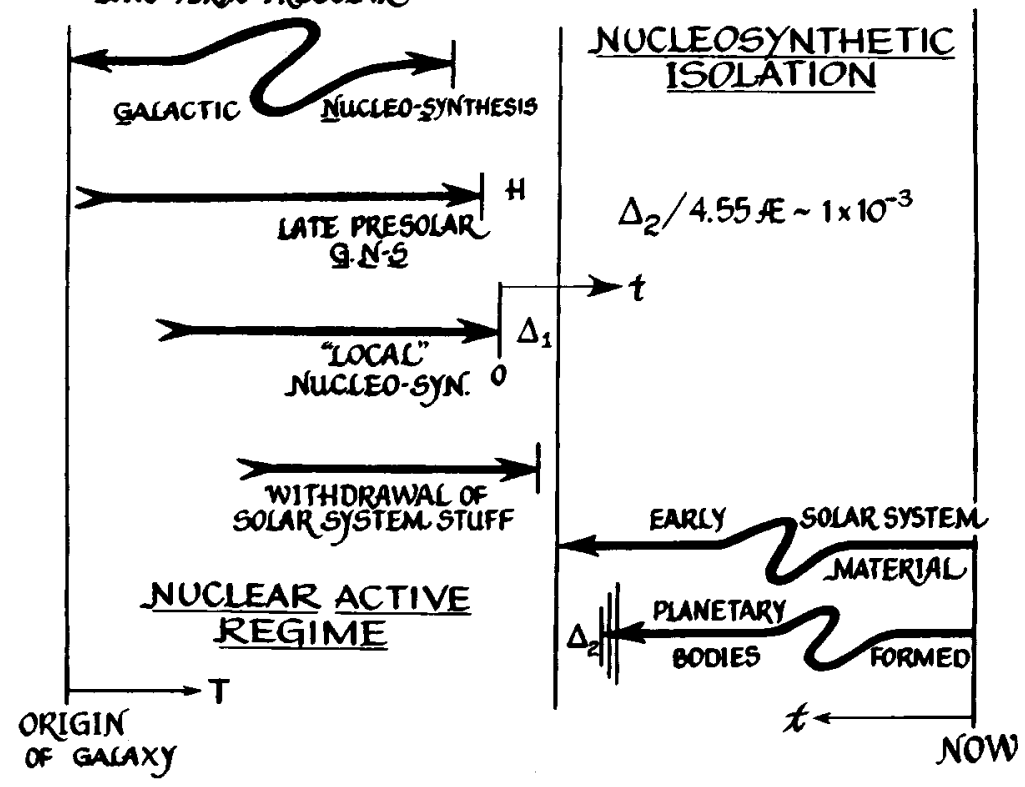

$\mathcal{B}$
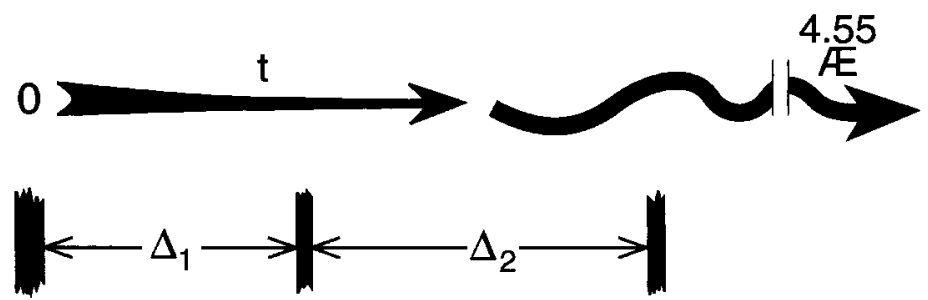

SYNTHESIS

CAI

P.D.

INJECTION

MIXING

I LIVE ${ }^{26}$ A I I CHONDRITE I
IACCUMULATION I

I ZONE

1 PLANETARY

IDIFFERENTION ! 
that ${ }^{26} \mathrm{Al}$ is an extremely potent heat source that would cause major melting and metamorphism even on small planetesimals that formed early (cf LaTourrette \& Wasserburg 1998). In comparing the observations with stellar-source models, it is imperative that possible differences in formation times are taken into account.

Table 1 and Figure 21 (see also Wasserburg et al 1996) show that ${ }^{244} \mathrm{Pu}$ (a pure $r$-process nuclide with a relatively long $\bar{\tau}$ ) is fully compatible with long-term uniform production (UP), much like all the longer-lived radioactivities. Hence, ${ }^{244} \mathrm{Pu}$ does not require a special stellar source close to the time of formation of the solar system. The only other information on transuranics is the upper bound on ${ }^{247} \mathrm{Cm}(\bar{\tau}=22.5 \mathrm{Myr})$, which should be present at the value $\left({ }^{247} \mathrm{Cm} /{ }^{235} \mathrm{U}\right) \sim$ $10^{-3}$ in the ESS. We note that determining ${ }^{247} \mathrm{Cm}$ from the variations in ${ }^{235} \mathrm{U} /{ }^{238} \mathrm{U}$ also requires estimates on the $\mathrm{Cm} / \mathrm{U}$ fractionation between phases. This factor is not well known. In the above hypothesis, all of the other nuclides would have substantial inventories in the ISM owing to long-term production. As long recognized by AGW Cameron (e.g. see Cameron 1993, Cameron et al 1993), this is the case for ${ }^{129} \mathrm{I}$, a pure $r$-process nuclide that cannot be produced in an AGB source. We not that the UP ratio of ${ }^{129} \mathrm{I} /{ }^{127} \mathrm{I}$ in Table 1 is far above the value observed; also ${ }^{107} \mathrm{Pd}(s+r)$ is overproduced. The pure $p$-process $($ or $\gamma, \mathrm{n})$ nuclide ${ }^{146} \mathrm{Sm}$ is instead compatible with long-term production. The same is true for ${ }^{53} \mathrm{Mn}$, which also may be associated with supernovae and cannot be produced in AGB stars. ${ }^{41} \mathrm{Ca}$ and ${ }^{26} \mathrm{Al}$ are underproduced by UP. The presence of the $r$-nucleus ${ }^{182} \mathrm{Hf}$ can be accounted for by UP, but this, together with the high ${ }^{129} \mathrm{I}$ production, requires that at least two $r$-process mechanisms are active, one producing the actinides and ${ }^{182} \mathrm{Hf}$, and the other explaining the lighter ${ }^{129} \mathrm{I}$ and ${ }^{107} \mathrm{Pd}$. This last process must be associated with rarer supernovae, to reconcile the high production with observations. The assignment of ${ }^{182} \mathrm{Hf}$ to long-term production requires that the replenishment of heavy $r$-process nuclei in molecular clouds must have a time scale of $\sim 10^{7}$ years (Wasserburg et al 1996).

Figure $19 a$. Schematic diagram showing the time scale and events of nucleosynthesis contributing to the solar nebula. The time from the origin of the galaxy to the last significant addition of nucleosynthetic material to the solar system is $T$. The time from the formation of the solar system and its state of isolation to the present is $4.55 \times 10^{9}$ years; this time is indicated in the figure as $4.55 \mathrm{AE}$, with $1 \mathrm{AE}=1 \times 10^{9}$ years. The period $T$ covers long-term and late-galactic nucleosynthesis (GNS). The first is responsible for the general inventory of nuclei. Late GNS events (i.e. supernovae) at time $H$ before the formation of the solar system are considered to be responsible for ${ }^{182} \mathrm{Hf}$ and ${ }^{53} \mathrm{Mn}$, whereas some local late-stage stellar source (supernovae, asymptotic giant branch star, Wolf Rayet) is responsible for injection of ${ }^{26} \mathrm{Al},{ }^{41} \mathrm{Ca},{ }^{107} \mathrm{Pd}$, and ${ }^{60} \mathrm{Fe}$ (after Wasserburg 1987). $b$. Schematic diagram showing the time scale and schedule of events after local late-stage injection. $\Delta_{1}$ is the interval between the last injection of freshly synthesized stellar materials and formation of calcium-aluminum-rich inclusions in the solar system. Note the range in time over which ${ }^{26} \mathrm{Al}$ is alive (up to $\sim 4 \times 10^{6}$ years). Here $\Delta_{2}$ is a time of planetary melting, differentiation, and crystallization. 


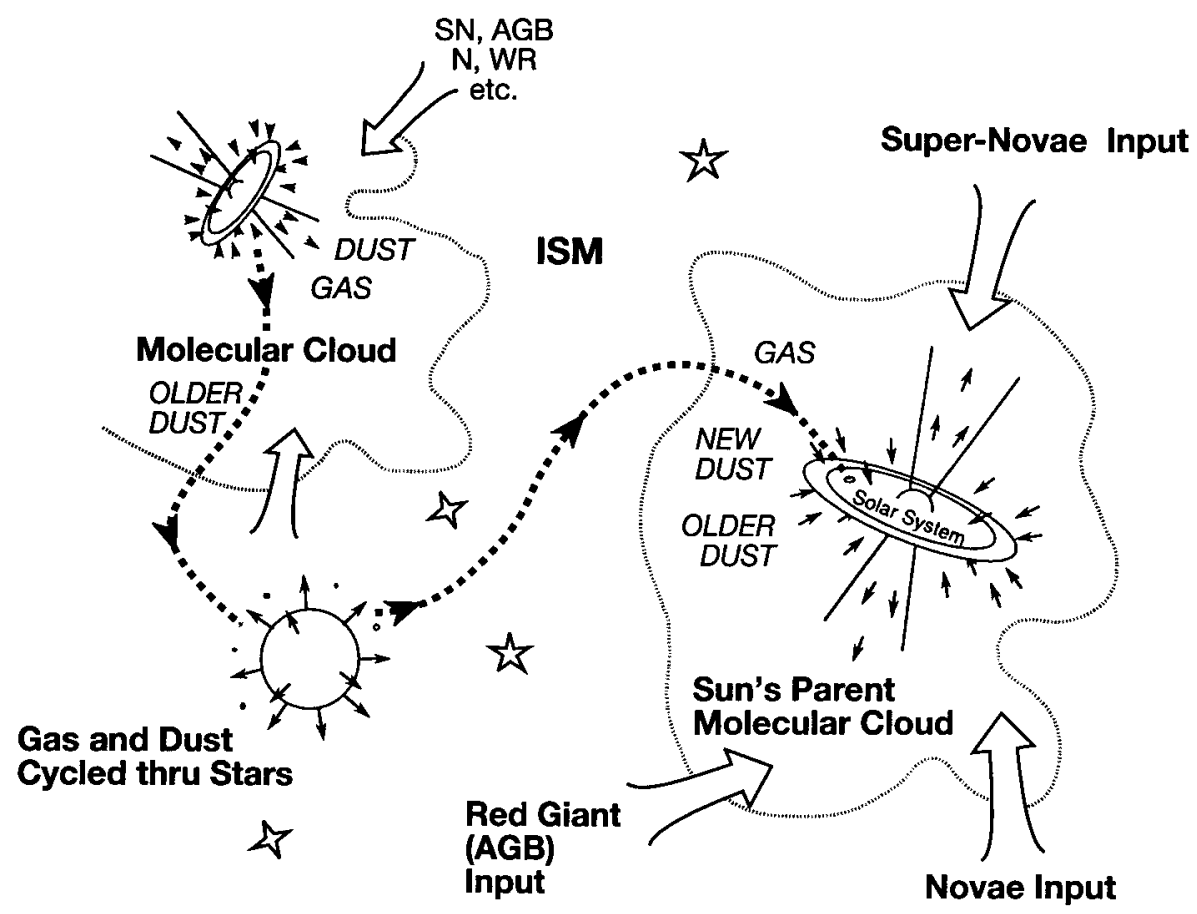

Figure 20 Injections of material from different stellar sources into a sequence of molecular clouds. The parent cloud of the protosun contains gas and preserved dust grains from several generations of stars formed in different molecular clouds, as well as the local inventory of short-, intermediate-, and long-lived nuclei produced over long time scales (after Huss GR, Nichols RH Jr, Wasserburg 1996, unpublished data).

In summary, some radioactive nuclei $\left({ }^{238,235} \mathrm{U},{ }^{232} \mathrm{Th},{ }^{244} \mathrm{Pu},{ }^{247} \mathrm{Cm}(?),{ }^{146} \mathrm{Sm}\right.$, ${ }^{129} \mathrm{I},{ }^{107} \mathrm{Pd},{ }^{60} \mathrm{Fe},{ }^{53} \mathrm{Mn}$, and ${ }^{182} \mathrm{Hf}$ ) can be understood in terms of UP from supernovae sources. However, some isotopes $\left({ }^{129} \mathrm{I}\right.$ and $\left.{ }^{107} \mathrm{Pd}\right)$ are overproduced. If UP is assumed for a single $r$-process-type site for both nuclei, then the solar system inventory of ${ }^{129} \mathrm{I}$ and ${ }^{107} \mathrm{Pd}$ would require a withdrawal of the ISM material from

Figure $21 a$. Graph of the log of the measured ratios (M) of the actinides ( $r$-process) relative to ${ }^{232} \mathrm{Th}$ and of ${ }^{146} \mathrm{Sm}$ ( $p$-process) relative to ${ }^{232} \mathrm{Th}$ in the early solar system as a function of their mean lifetimes. $\bar{\tau}_{i}$ is the reciprocal of their decay constant. The straight line is a reference line corresponding to unit production ratios for all species. UP are the calculated values using best estimates of the relative production rates $\left(P_{i}\right)$ for $T=10 \mathrm{AE}\left(1 \mathrm{AE}=10^{9}\right.$ years $) . S S P$ corresponds to the steady-state case using $\left(P_{i}\right)$ values. The ${ }^{247} \mathrm{Cm}$ is an upper bound (Wasserburg et al 1996). $b$. Same as $a$ but for mean lives $<2 \times 10^{8}$ years; these include the $r$-process nuclei ${ }^{182} \mathrm{Hf},{ }^{129} \mathrm{I}$, and ${ }^{107} \mathrm{Pd}$, as well as ${ }^{26} \mathrm{Al}$. Note that ${ }^{129} \mathrm{I}$ and ${ }^{107} \mathrm{Pd}$ for UP are far above the measured points. The upper right corner corresponds to the normalization point for ${ }^{232} \mathrm{Th}$. 

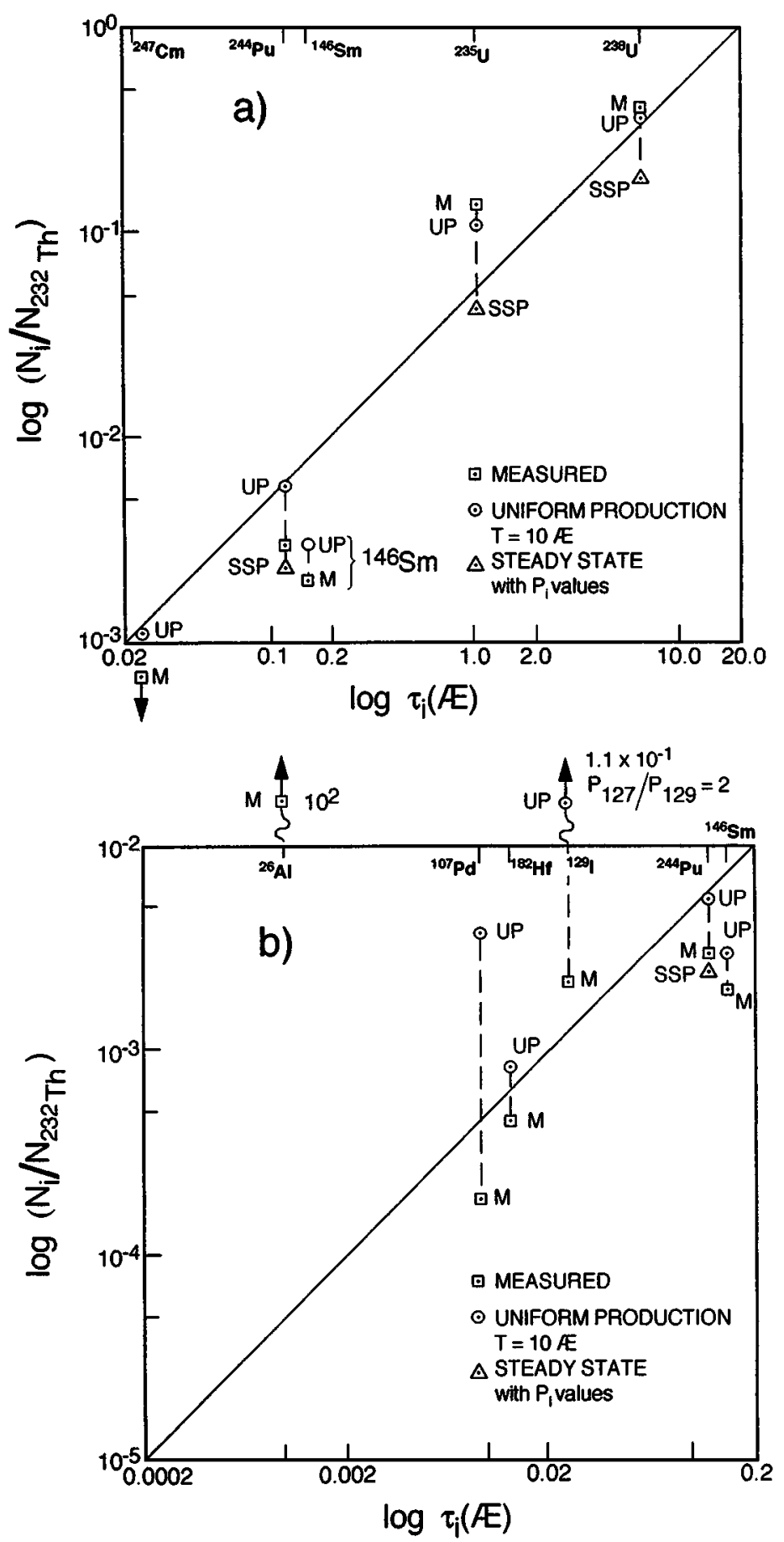
further fresh addition for $\sim 10^{8}$ years, which has long been recognized as a requirement of ${ }^{129} \mathrm{I} /{ }^{127} \mathrm{I}=10^{-4}$ (cf Wasserburg et al 1960, Schramm \& Wasserburg 1970). This is in conflict with ${ }^{182} \mathrm{Hf}$ and ${ }^{53} \mathrm{Mn}$ data. We follow the approach of Wasserburg et al (1996) for the model of two sites producing $r$-process nuclei at different rates. Other non- $r$-process nuclei that can be produced in supernovae (e.g. ${ }^{26} \mathrm{Al}$ ) are inadequately contributed by UP. There are thus three distinct issues: how can the observations on nuclides produced solely in supernovae be reconciled with theoretical models; what is the role that an AGB star may play in providing some of the nuclei; and how can rapid addition of freshly synthesized nuclei be achieved in a physically reasonable scenario?

\subsection{The Asymptotic Giant Branch Contamination Model}

It is assumed that a mass of stellar ejecta is well mixed with some part of a molecular cloud and that the solar nebula was formed from this material. We assume that both the stellar ejecta and cloud each have their own uniform composition. The composition of the ejecta is determined by the stellar model and by the time scale between production $(t=0)$ and the formation of an object from the solar mix $(t=\Delta)$. For reference, we take the formation of CAIs to be $\Delta_{1}$ and of later objects to be $\Delta_{1}+\Delta_{2}$ (Figure 19). If the ejection and mixing were instantaneous, then, in the mixture (mix), the ratio of a short-lived radioactive nuclide $\mathrm{R}$ to the net number of stable nuclei $\mathrm{S}$ (of the same element) is given by $\left(N_{R}{ }^{0} / N_{S}\right)^{m i x} \equiv \alpha_{R, S}=N_{R}{ }^{A G B} /\left(N_{S}{ }^{0}+N_{S}{ }^{A G B}\right)$. Here $N_{S}{ }^{0}$ and $N_{S}{ }^{A G B}$ are the numbers of $S$ nuclei per gram in the molecular protosolar cloud (SC) and the AGB ejecta, respectively. If the contribution of the stable nuclide $S$ from the star to the cloud is small compared with the amount in the protosolar cloud SC, we obtain $\alpha_{R, S} \approx\left(N_{R} / N_{S}\right)^{A G B} q_{S}{ }^{A G B} M_{A G B} / q_{S}{ }^{S C} M_{S C}$. Here $q_{S}{ }^{S C}$ and $q_{S}{ }^{A G B}$ are the numbers of $\mathrm{S}$ nuclei per gram in the cloud and in the AGB ejecta, respectively. It is evident that the ratios $N_{R} / N_{S}$ in the envelope of the star and the yields ( $q$ 'S) of stable nuclei S in the star's ejecta compared with that in the ambient ISM are the governing factors. A self-consistent scenario would require that the dilution factor $M_{A G B} / M_{S C}$ be the same for all isotope pairs. If we consider only nuclei produced in the He shell (or the $\mathrm{H}$ shell), then $M_{A G B}$ should simply refer to the mass of He-intershell material $\left(M_{\mathrm{He}}\right)$ or the mass of H-shell material $\left(M_{\mathrm{H}}\right)$ cumulatively dredged up into the envelope, and the values of the other ratios are computed for the same stellar layers (e.g., $q_{S}{ }^{\mathrm{He}}$ and $N_{R}{ }^{\mathrm{He}} / N_{S}{ }^{\mathrm{He}}$ ). If an object forms at time $\Delta$ after the production event, then the $\alpha_{R, S}$ becomes $\alpha_{R, S}(\Delta)=\alpha_{R, S}(0) e^{-\Delta / \bar{\tau}_{R}}$. The term $q_{S}{ }^{A G B} / q_{S}{ }^{S C}\left(\equiv q_{S} / q_{S}{ }^{0}\right)$ is $>1$ if $S$ is produced in the star and $<1$ if $S$ is destroyed. We note that, for a given stellar model, the values of $q_{S} / q_{S}{ }^{0}$ may range over several orders of magnitude for different species $S$; the same is true for the term $\exp \left(-\Delta / \bar{\tau}_{R}\right)$ for different species R. To compare the data on short-lived nuclei in the ESS to any AGB model, we must first establish a dilution factor for one pair of nuclei and then compare the results for all others. Because the formation time $\Delta$ is critical, we have chosen the pair with well-defined relative production characteristics and with a lifetime that is sufficiently long so that an uncertainty 
in $\Delta$ will not seriously alter the estimate of the dilution factor. For this reason, the pair ${ }^{107} \mathrm{Pd}\left(\bar{\tau}=9 \times 10^{6}\right.$ years $)$ and ${ }^{108} \mathrm{Pd}$ were chosen for $\Delta_{1}+\Delta_{2}=5 \times 10^{6}$ years (see Wasserburg et al 1994, 1995).

\subsection{Asymptotic Giant Branch Sources of Short-Lived Nuclei}

Results of Previous Models Estimating the possible contributions from different stellar sources is dependent on the existence of adequate stellar models. Detailed stellar models of $s$-process nucleosynthesis have been developed, based on a prescription for the ${ }^{13} \mathrm{C}$ neutron source and activation of the ${ }^{22} \mathrm{Ne}$ neutron source as determined by the internal temperature structure. Although there are serious concerns with the actual mechanisms governing the formation of the ${ }^{13} \mathrm{C}$ neutron source, the general AGB results appear to be consistent with observations and have provided a good guide for their interpretation (see Sections 5 and 6). The $s$-process calculations are based on stellar models that follow the evolution of a star through the TP-AGB phase. The nucleosynthetic results are, of course, dependent on the specific nuclear-reaction rates. The stellar structure, instead, is not governed by the minor energetic contributions from neutron production and $s$ processing. It follows that the fundamental, independent parameters determining the production of short-lived nuclei for the TP-AGB phase are initial stellar mass and composition (metallicity $Z$ ), mass loss rates, and the magnitude of the ${ }^{13} \mathrm{C}$ pocket that is assumed. Note that AGB sources have well-mixed envelopes, which is not the case for supernovae. There have been some efforts at evaluating the yields of a large number of radioactive products in a self-consistent fashion for AGB stars, which lead to clear predictions that appear to be reasonably reliable.

Previous estimates of some $s$-process species were made by using a simplified steady-flow approximation for the neutron exposure and a constant neutron density (cf Cameron 1993). Isotopic ratios for a single element for steady-state flow patterns provided an excellent guide. There are also significant contributions from non-s-process isotopes in AGB stars. Forestini et al (1991) evaluated the production mechanism of ${ }^{26} \mathrm{Al}$ in the $\mathrm{H}$ shell and showed that this could contribute significantly to the ${ }^{26} \mathrm{Al}$ inventory. However, no self-consistent yields for different isotopes were obtained by this approach, nor were the destruction mechanisms properly considered. If one is to consider late contributions of an AGB star to the protosolar nebula, then it is clear that a comprehensive analysis is required that follows AGB evolution and takes the net production (including destruction) from $\mathrm{H}$ burning and $\mathrm{He}$ burning in a star. One has also to consider the dilution effects of mixing nuclear-processed matter to the surface through TDU and the final losses to the ISM through stellar winds.

A first effort in this direction (Wasserburg et al 1994) was the calculation of AGB yields for $Z=Z_{\odot}=0.02$ and for masses from 1.5 to $3 M_{\odot}$ from the models then available (see Section 4). A series of yields were obtained for a wide variety of isotopes. Results for the production ratios were not significantly sensitive to the stellar mass, but the yields of the different isotopes depended 
critically on the extent of neutron exposure and neutron density. Those nuclei produced by an AGB star could be reasonably estimated, and others were clearly excluded. For ${ }^{26} \mathrm{Al}$, a time scale $\Delta_{1} \approx 10^{6}$ years was found. The nuclide ${ }^{26} \mathrm{Al}$ is produced in the H-burning shell, but it was also found to be extensively destroyed in the $\mathrm{He}$ shell. In the $\mathrm{H}$ shell, the main production process of ${ }^{26} \mathrm{Al}$ is through ${ }^{25} \mathrm{Mg}(\mathrm{p}, \gamma){ }^{26} \mathrm{Al}$. The rate for the competing reaction ${ }^{26} \mathrm{Al}(\mathrm{p}, \gamma){ }^{27} \mathrm{Si}$ is still uncertain (Arnould et al 1995) and constitutes the main problem for estimating the ${ }^{26} \mathrm{Al}$ production. The ${ }^{26} \mathrm{Al}$ nuclei surviving $\mathrm{p}$ captures sink into the He zone with H-burning ashes. Here they are efficiently destroyed by ${ }^{26} \mathrm{Al}(\mathrm{n}, \mathrm{p})^{26} \mathrm{Mg}$ and by ${ }^{26} \mathrm{Al}(\mathrm{n}, \alpha)^{23} \mathrm{Na}$ reactions, because the total neutron capture cross section of ${ }^{26} \mathrm{Al}$ is 385 mbarn in the temperature conditions of a convective pulse. Somewhat surprisingly, it was found that AGB models could contribute ${ }^{60} \mathrm{Fe}$. Production of ${ }^{60} \mathrm{Fe}$ requires substantial neutron densities and occurs during the high-neutron-density peak in the convective He zone, owing to the ${ }^{22} \mathrm{Ne}$ neutron source. ${ }^{60} \mathrm{Fe}$ is fed through the minor $(\mathrm{n}, \gamma)$ channel on ${ }^{59} \mathrm{Fe}(\bar{\tau}=65.1 \mathrm{~d})$, which is not affected by the temperature in the He zone (Takahashi \& Yokoi 1987). Once produced, ${ }^{60} \mathrm{Fe}$ does not suffer appreciable destruction by neutron captures because of its low cross-section ( $\sigma_{60} \simeq 3.2 \mathrm{mbarn}$ ). Agreement was obtained for ${ }^{60} \mathrm{Fe} /{ }^{56} \mathrm{Fe}$, assuming $\Delta_{1}+\Delta_{2} \leq 5 \times 10^{6}$ years. Upon the discovery of ${ }^{41} \mathrm{Ca}\left(\bar{\tau}=0.15 \times 10^{6}\right.$ years $)$, it was found that this datum could also be matched by the same AGB source if $\Delta_{1} \approx\left(5 \times 10^{5}\right)-\left(7 \times 10^{5}\right)$ years (Wasserburg et al 1995). As in the case of ${ }^{60} \mathrm{Fe}$, ${ }^{41} \mathrm{Ca}$ is produced by ${ }^{40} \mathrm{Ca}(\mathrm{n}, \gamma){ }^{41} \mathrm{Ca}$ when the ${ }^{22} \mathrm{Ne}$ source is activated. Because ${ }^{40} \mathrm{Ca}$ has no precursor that is fed by neutron captures, production of ${ }^{41} \mathrm{Ca}$ depends on the initial ${ }^{40} \mathrm{Ca}$ abundance (i.e. $Z$ ). ${ }^{41} \mathrm{Ca}$ has a high neutron capture cross-section (560 mbarn) undergoing the reactions ${ }^{41} \mathrm{Ca}(\mathrm{n}, \alpha){ }^{38} \mathrm{Ar}$ and ${ }^{41} \mathrm{Ca}(\mathrm{n}, \mathrm{p}){ }^{41} \mathrm{~K}$. Hence we have the ratio ${ }^{41} \mathrm{Ca} /{ }^{40} \mathrm{Ca} \approx \sigma_{40} / \sigma_{41} \approx 10^{-2}$ (Cameron 1993). Note that the mean life of ${ }^{41} \mathrm{Ca}$ for $\mathrm{e}^{-}$capture is longer in stellar conditions than in the laboratory (by 1 order of magnitude, see Fuller et al 1985). This means that ${ }^{41} \mathrm{Ca}$ is virtually stable in the He shell and through most of the envelope, so that its decay before ejection is small. The results for ${ }^{129} \mathrm{I}$ and ${ }^{53} \mathrm{Mn}$ showed that these nuclei could not be produced in an AGB source, in agreement with earlier calculations by Cameron (1993). For ${ }^{129} \mathrm{I}$, the very low yield is caused by the low neutron density and the branching at ${ }^{128} \mathrm{I}$. For ${ }^{53} \mathrm{Mn}$, there are no channels feeding this nuclide during the neutron exposure. A good quantitative agreement was obtained between some of the observed isotopic ratios and those for a late AGB injection. This accord was obtained for the nuclides ${ }^{107} \mathrm{Pd},{ }^{26} \mathrm{Al},{ }^{60} \mathrm{Fe}$, and ${ }^{41} \mathrm{Ca}$ by matching the dilution factor $M_{A G B} / M_{S C}$ and the neutron exposure $\left(\tau_{0}\right)$.

From the above results it was suggested that a late-stage injection from an AGB star of $\sim 3 M_{\odot}$ into the protosolar cloud could provide the inventory of ${ }^{107} \mathrm{Pd},{ }^{26} \mathrm{Al},{ }^{60} \mathrm{Fe}$, and ${ }^{41} \mathrm{Ca}$ in the early solar system with a dilution factor of $M_{\mathrm{He}} / M_{S C}=1.46 \times 10^{-4}$ for a low neutron exposure $\left(\tau_{0}=0.03 \mathrm{mbarn}^{-1}\right)$. The corresponding ratio of the mass of the AGB envelope to the contaminated solar cloud was $M_{A G B} / M_{S C} \sim 10^{-2}$. The choice of 3-M $M_{\odot}$ models was based on obtaining adequate ${ }^{60} \mathrm{Fe}$ production. This scenario required that the time between 


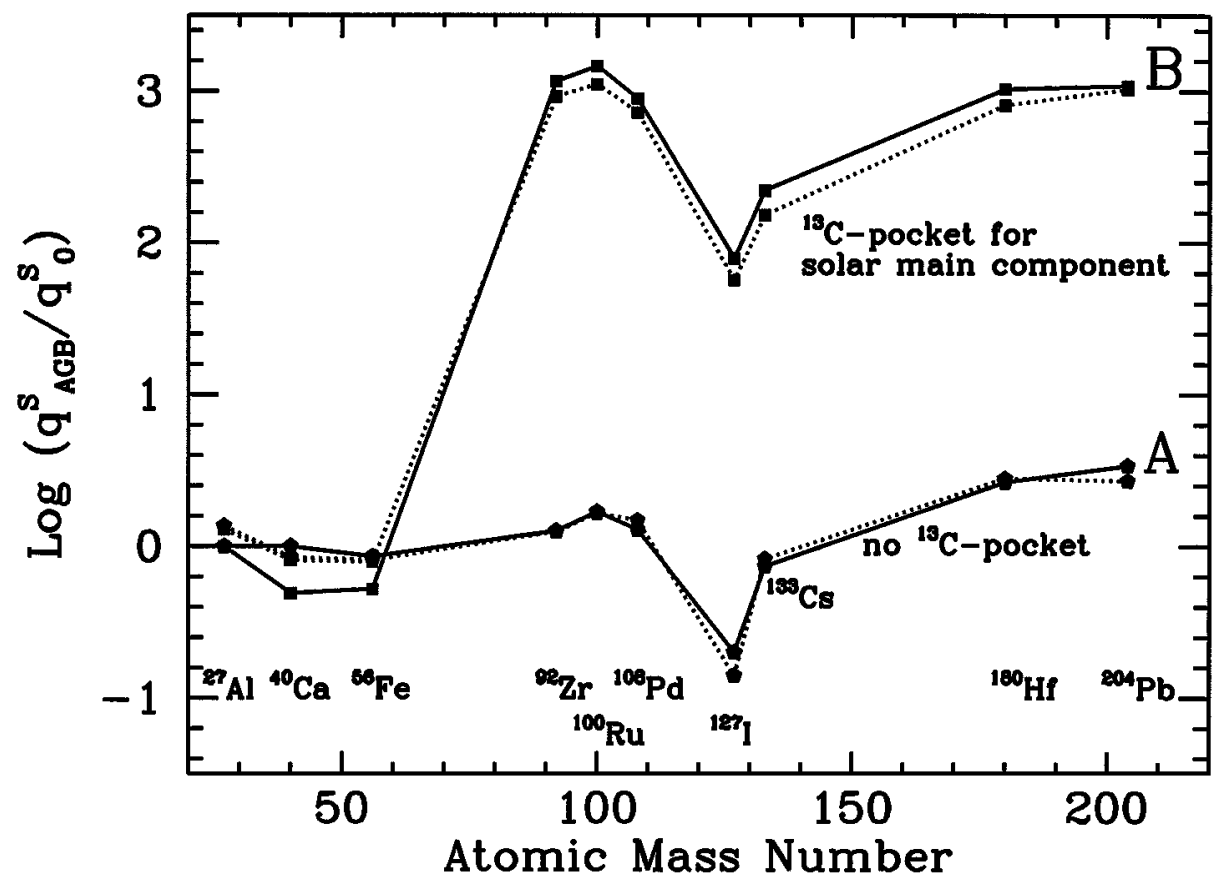

Figure 22 Logarithm of the enrichment factors of a slow neutron capture $s$-process nuclide $\mathrm{S}$ from a model asymptotic giant branch (AGB) source relative to its original abundance as a function of atomic mass number for selected nuclides. Full curves $(a)$ are for earlier models with $\tau_{0}=0.03$ and 0.28 mbarn $^{-1}$, respectively. Dotted curves $(b)$ are for new models, either without a carbon pocket or with the choice standard (ST) for it discussed in the text. Note that AGB models with $\log \left(q_{A G B}{ }^{s} / q_{o}{ }^{s} \approx 1\right)$ appear to provide ${ }^{26} \mathrm{Al},{ }^{41} \mathrm{Ca},{ }^{60} \mathrm{Fe}$, and ${ }^{107} \mathrm{Pd}$, whereas all models with ${ }^{13} \mathrm{C}$ pockets overproduce the heavy nuclei compared with the light ones so that no concordant solution is possible.

production and injection and the formation of the earliest solar system condensates be very small $\left(0.5-0.7 \times 10^{6}\right.$ years $)$. This time interval places strong dynamical constraints on the injection, mixing, and collapse of the protosolar cloud. The basic requirement on the model AGB star was that the neutron exposure would have to be low $\left(\tau_{0}=0.03 \mathrm{mbarn}^{-1}\right)$ as compared with $\tau_{0}=0.28 \mathrm{mbarn}^{-1}$, which is the value used to obtain a good reproduction of the solar main $s$-process component in this class of models. The low value of $\tau_{0}$ was necessary owing to the dependence of the yields on the neutron exposure. From Figure 22 it can be seen that stellar models with high values of $\tau_{0}$ produce large amounts of ${ }^{107} \mathrm{Pd}$ and low amounts of ${ }^{26} \mathrm{Al},{ }^{60} \mathrm{Fe}$, and ${ }^{41} \mathrm{Ca}$. The low value of $\tau_{0}$ corresponds to the minimum neutron exposure generated by the ${ }^{22} \mathrm{Ne}$ source (plus a marginal contribution from the low ${ }^{13} \mathrm{C}$ abundance in the $\mathrm{H}$ shell ashes); no ${ }^{13} \mathrm{C}$ pocket was assumed by Wasserburg et al (1995). 
The AGB models also provided explicit predictions of other radioactive species. The prediction of ${ }^{182} \mathrm{Hf} /{ }^{180} \mathrm{Hf}$ was of particular interest. Lee \& Halliday (1995) and Harper \& Jacobsen (1994) discovered ${ }^{182} \mathrm{~W}$ deficiencies in iron meteorites. It was further demonstrated by Lee \& Halliday (1996) that there was a widespread occurrence of the ${ }^{182} \mathrm{~W}$ isotopic effects. The observed value of ${ }^{182} \mathrm{Hf} /{ }^{180} \mathrm{Hf}$ was a factor of 100 greater than the predicted value from the AGB model. This was true even though $\sim 43 \%$ of ${ }^{182} \mathrm{~W}$ is produced by the $s$ process caused by the branching at ${ }^{181} \mathrm{Hf}$. The high $\left({ }^{182} \mathrm{Hf} /{ }^{180} \mathrm{Hf}\right)_{E S S}$ has been explained (Wasserburg et al 1996) by showing that the ${ }^{182} \mathrm{Hf}$ abundance, as well as ${ }^{53} \mathrm{Mn}$, is a consequence of the UP model (see Table 1). Thus, like the actinides, ${ }^{182} \mathrm{Hf}$ and ${ }^{53} \mathrm{Mn}$ are considered to be present in the inventory of the precursor ISM owing to long-time-scale production by supernovae. The ambient ISM is not a reasonable source for ${ }^{41} \mathrm{Ca}$, because its abundance would require a time scale of $<3 \times 10^{5}$ years for $\Delta$. However, as noted earlier, this model requires that the usual assumption of a single $r$ process must be rejected. The question of the diversity of $r$-processes is now the subject of vigorous investigation of the abundances of elements in very low metallicity $([\mathrm{Fe} / \mathrm{H}]<-2)$ stars. If the hypothesis of two distinct $r$-process sources for the two $r$-abundance peaks (and the inferred time scales) is valid, then this could be used to identify the first generation of stars with $r$ nuclei in the Galaxy. It is necessary to have relative quantitative abundances of $r$-elements at both of the $r$-process peaks to test this model (Sneden et al 1998, Crawford et al 1998, Cowan et al 1999). This problem is both technically and observationally very demanding. The possibility of multiple $r$-processes is a fundamental one. If the hypothesis adopted here is not valid, there are major problems with regard to our understanding of the short- and intermediate-lived nuclei found in the early solar system. In addition to the optical observations, there are proposed studies using both gamma-rays and X-rays to study $r$-process sites (Diehl \& Timmes 1998, Qian et al 1998).

New Generation Models We now consider a new set of calculations of yields for short-lived nuclei based on the advances in stellar models outlined in Section 5 , using recently revised reaction rates. In these calculations, the abundances in the various stellar layers (He intershell, $\mathrm{H}$ shell, and envelope) were followed in detail, based on the prescriptions given by the new stellar models for processes like dredge-up and mass loss. This allowed us to obtain a precise estimate of the relative mass contributions of the reservoirs producing $\mathrm{n}$-rich radioactive nuclei (e.g. ${ }^{60} \mathrm{Fe}$ and ${ }^{107} \mathrm{Pd}$ ) and the regions producing ${ }^{26} \mathrm{Al}$. As for the contribution to ${ }^{26} \mathrm{Al}$ that comes from the $\mathrm{H}$ shell, it has a rather complex fate, a large fraction of it being cycled and partially destroyed in the He shell. For this reason, in the new model we present results on the production of ${ }^{26} \mathrm{Al}$ split into two parts by whether it is injected into the AGB winds either directly from the $\mathrm{H}$-burning reservoir (either $\mathrm{H}$ shell or $\mathrm{HBB}$ ) or after partial depletion in the He shell. Concerning ${ }^{26} \mathrm{Al}$, note that there is a very large experimental uncertainty in the reaction rate for ${ }^{26} \mathrm{Al}(\mathrm{p}, \gamma)^{27} \mathrm{Mg}$ (Arnould et al 1995). On the whole, any difference between the model and the ESS values of less than a factor of 2 should not be considered critical. The results 
are now discussed in terms of the following parameters: $(a)$ the efficiency of the ${ }^{22} \mathrm{Ne}$ source, which is essentially given by the stellar model, $(b)$ the magnitude of the ${ }^{13} \mathrm{C}$ pocket, and $(c)$ the initial mass and metallicity. The effectiveness of ${ }^{22} \mathrm{Ne}$ burning is controlled by the maximum bottom temperature $T_{b}$, and is dependent on the stellar mass and $Z$. As discussed in Sections 3, 4, and 5, in low-mass stars $T_{b} \sim 3 \times 10^{8} \mathrm{~K}$, and the ${ }^{22} \mathrm{Ne}$ source is only marginally activated; in more massive AGBs, $T_{b}$ increases up to $\sim 3.5 \times 10^{8} \mathrm{~K}$, and the ${ }^{22} \mathrm{Ne}$ source is very effective. As for the ${ }^{13} \mathrm{C}$ source, in most cases discussed here, we did not introduce a ${ }^{13} \mathrm{C}$ pocket, because Wasserburg et al $(1994,1995)$ found only models with very low $s$-process exposures to be consistent with the record of extinct radioactivities in the ESS. This was confirmed with calculations of some new generation models (see Figure 22). In the calculations listed in Table 2, the neutron sources were within the convective $\mathrm{He}$ pulse and consisted of the ${ }^{22} \mathrm{Ne}$ source and the limited ${ }^{13} \mathrm{C}$ ingested into the pulse from $\mathrm{H}$-burning ashes.

Table 2 shows the results for a model of $1.5 M_{\odot}$. The value of $\mathrm{d}_{0}$ corresponds to the dilution factor calculated as in Wasserburg et al (1994), using the net ${ }^{107} \mathrm{Pd}$ produced in the $\mathrm{He}$ intershell region (in this case, the ${ }^{107} \mathrm{Pd}$ produced both during the radiative burning in the ${ }^{13} \mathrm{C}$ pocket and the contribution from the intershell convective pulse). The ratio $\mathrm{M}_{\mathrm{E}} / \mathrm{M}_{\mathrm{He}}$ is the ratio of the mass of the convective envelope to the net contribution of mass from the He intershell after repeated dredge-up when the star reaches $\mathrm{C} / \mathrm{O}$ about unity. $\mathrm{M}_{\mathrm{H}} / \mathrm{M}_{\mathrm{He}}$ is the ratio of the masses of the contributions from the $\mathrm{H}$-shell to those from the He-intershell. The ${ }^{26} \mathrm{Al} /{ }^{27} \mathrm{Al}$ ratio in the $\mathrm{H}$-shell and the ${ }^{26} \mathrm{Al} /{ }^{27} \mathrm{Al}$ in the He-intershell are listed in Table 2. The values calculated for the early solar system at different times represent the total from these 2 sources $(\mathrm{H}$-shell $+\mathrm{He}$-intershell). These results were obtained by starting from the model by Straniero et al (1997), in which TDU was found, but mass loss was not included. For the present calculations, the envelope evolution was monitored through a post-processing phase in which we introduced a mass loss with the Reimer's (1975) formula using $\eta=0.5$. (For all the other stellar models discussed here, TDU was self-consistently produced in the stellar models, and mass loss was included from the beginning of the calculation.) These results (Table 2) give production ratios and $q_{S} / q_{S}{ }^{0}$ values that are very similar to those of the previous-generation models for $\tau_{0}=0.03 \mathrm{mbarn}^{-1}$. As in that case, the abundances of the short-lived nuclei are also in good agreement with the observed ESS values. There is an excess of ${ }^{41} \mathrm{Ca}$ (5.7-fold) with $\Delta_{1}=0.5 \mathrm{Myr}$, which could readily be accommodated if $\Delta_{1}=0.7 \mathrm{Myr} .{ }^{60} \mathrm{Fe}$ production is increased but remains, as before, dependent on $\Delta_{1}+\Delta_{2}$ and the corresponding value of $\left({ }^{60} \mathrm{Fe} /{ }^{56} \mathrm{Fe}\right)_{P D}$, where the subscript PD is for planetary differentiate, as well as on the uncertainty in the neutron capture cross-section of its precursor ${ }^{59} \mathrm{Fe}$ (of up to a factor of 2$)$ and on the ${ }^{22} \mathrm{Ne}(\alpha, \mathrm{n})^{25} \mathrm{Mg}$ rate. We also found that, for a $1.5-M_{\odot}$ model and $Z=Z_{\odot} / 2$, the results are essentially unchanged. There is thus no requirement that a polluting AGB star be initially of solar composition.

Models at $3 M_{\odot}$ were explored for the case of no ${ }^{13} \mathrm{C}$ pocket. The results are again roughly commensurate with those found for $1.5 M_{\odot}$. However, the ${ }^{60} \mathrm{Fe}$ is 


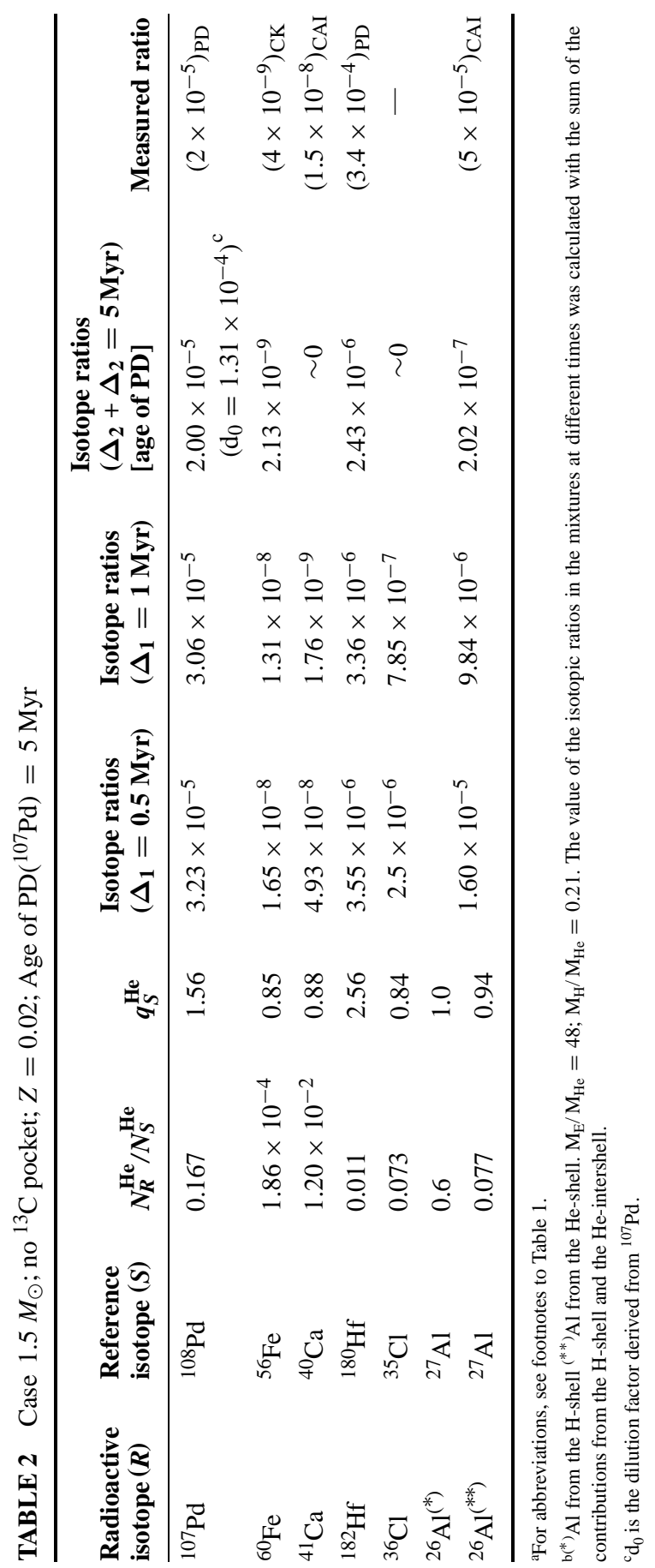


enhanced by a factor of 5.5 owing to the higher $T_{b}$ in the pulses. The difference in the ${ }^{60} \mathrm{Fe}$ yields compared with the earlier model is principally due to the somewhat higher temperatures obtained for the convective pulse region in the new model and to the increased rate for ${ }^{22} \mathrm{Ne}(\alpha, \mathrm{n}){ }^{25} \mathrm{Mg}$ reaction (Denker et al 1995). ${ }^{26} \mathrm{Al}$ is down by a factor of 5 owing to the narrowing of the $\mathrm{H}$ shell with increased stellar mass, so that production of ${ }^{26} \mathrm{Al}$ appears to be more difficult in this case. ${ }^{41} \mathrm{Ca} /{ }^{40} \mathrm{Ca}$ is high by a factor of 5.5 and can be readily accommodated by taking $\Delta_{1}=0.7 \mathrm{Myr}$. Any ${ }^{13} \mathrm{C}$ pocket introduced would grossly overproduce ${ }^{107} \mathrm{Pd}$, and no match would be obtained for the other observations.

Figure 23 shows the $N_{R} / N_{S}$ ratios in the envelope for ${ }^{26} \mathrm{Al}$ and ${ }^{107} \mathrm{Pd}$ for the models of a 1.5- and of a 3- $M_{\odot}$. The ratios are plotted together with the $\mathrm{C} / \mathrm{O}$ ratio as a function of fractional envelope mass lost owing to stellar winds. The vertical line indicates the point at which a $\mathrm{C}$ star $(\mathrm{C} / \mathrm{O}=1)$ is formed. The ${ }^{26} \mathrm{Al} /{ }^{27} \mathrm{Al}$ ratios achieved in the envelope match typical values measured in circumstellar $\mathrm{SiC}$ grains recovered from meteorites (see e.g. Zinner 1997).

We also explored models of higher AGB masses that were experiencing HBB, because this is known to give a potentially high contribution to ${ }^{26} \mathrm{Al}$ (Lattanzio et al 1996, Frost et al 1998). We computed HBB for a $7-M_{\odot}$ star and $Z_{\odot}$ through a post-processing code, using the stellar parameters from the complete stellar model. At this mass, we considered two cases - one without and one with a ${ }^{13} \mathrm{C}$ pocket. In the latter case, we used a ${ }^{13} \mathrm{C}$ pocket that was found necessary to fit the observed abundances of some post-AGB stars that are probably descendants of IMS (e.g. see Decin et al 1998). The ${ }^{13} \mathrm{C}$ pocket was introduced in this massive AGB (Table 3) to compensate for the high $T_{b}$ at the bottom of the pulses, which induces a high neutron density and, hence, a high ${ }^{60} \mathrm{Fe}$ abundance. Neutrons from the ${ }^{13} \mathrm{C}(\alpha, \mathrm{n}){ }^{16} \mathrm{O}$ reaction, indeed, increase the abundance of ${ }^{107} \mathrm{Pd}$ at low $\mathrm{n}$ density without affecting ${ }^{60} \mathrm{Fe}$. The values of $N_{R}{ }^{\mathrm{He}} / N_{S}{ }^{\mathrm{He}}$ for all species are very similar to what was found in lower stellar masses; however, $q_{S}{ }^{\mathrm{He}} / q_{S}{ }^{0}$ is high for ${ }^{108} \mathrm{Pd}$ and $\left(q_{S}{ }^{\mathrm{He}} / q_{S}{ }^{S C}=103\right)$ when a ${ }^{13} \mathrm{C}$ pocket (ST case) is included. ${ }^{26} \mathrm{Al}$ is produced in high abundance by HBB. Here the ${ }^{26} \mathrm{Al}$ in Table 3 is produced in the envelope by HBB. The ratio of the mass of the envelope $\left(\mathrm{M}_{\mathrm{E}}\right)$ to the mass of the cumulatively dredged-up material from the He-intershell $\left(\mathrm{M}_{\mathrm{He}}\right)$ is high. Due to the fairly high neutron exposure in the He-intershell, all of the ${ }^{26} \mathrm{Al}$ initially present in the H-burning ashes is completely consumed in the case of the $5 M_{v}$ (see Table 3 ). ${ }^{60} \mathrm{Fe}$ is especially high, as shown in Table 3, but could possibly be accomodated with a large $\Delta_{1}+\Delta_{2}$ (not shown). In both cases (with and without a ${ }^{13} \mathrm{C}$ pocket), a $7-M_{\odot}$ AGB source is excluded if we use the underproduction of ${ }^{41} \mathrm{Ca}$ as a criterion.

In summary, both the new-generation results and the earlier calculations require a very small ${ }^{13} \mathrm{C}$ pocket if an AGB is to roughly explain the observed abundances of ${ }^{41} \mathrm{Ca},{ }^{26} \mathrm{Al},{ }^{60} \mathrm{Fe}$, and ${ }^{107} \mathrm{Pd}$ in the ESS. There is a clear overall self-consistency in the results that have been obtained with the new model and the earlier calculations, and we consider that they are robust. The basis for rejecting any of the AGB models must rest on the very low relative yields of some nuclei with ${ }^{107} \mathrm{Pd} /{ }^{108} \mathrm{Pd}$ as the primary reference. The ratio and yields of ${ }^{107} \mathrm{Pd} /{ }^{108} \mathrm{Pd}$ are by far the least 

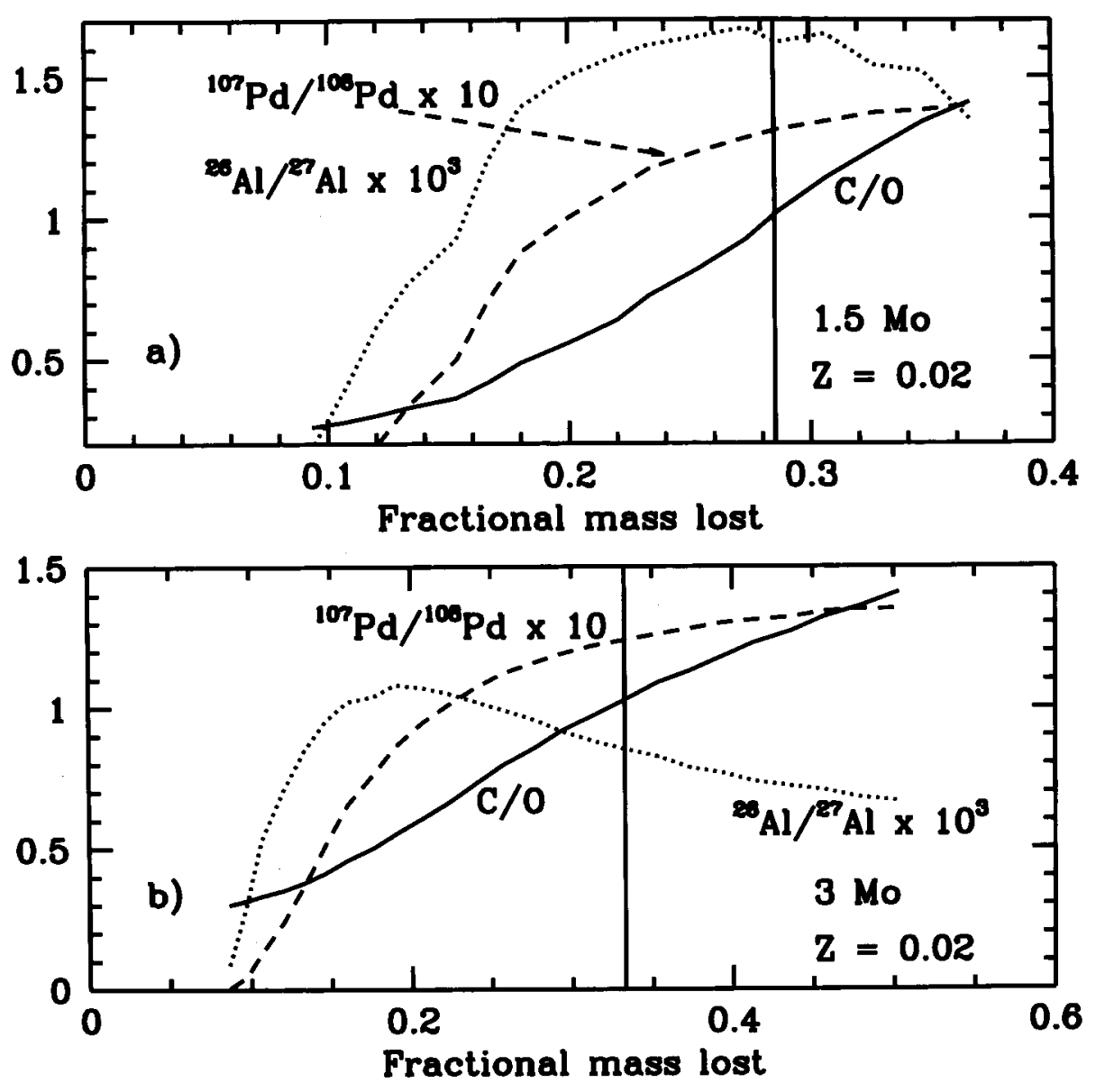

Figure 23 Ratios $N_{D} / N_{R}$ for ${ }^{26} \mathrm{Al}$ and ${ }^{107} \mathrm{Pd}$ in the envelope of $(a)$ the $1.5-M_{\odot}$ asymptotic giant branch (AGB) model and $(b)$ the $3-M_{\odot}$ AGB model of solar metallicity. No ${ }^{13} \mathrm{C}$ pocket was assumed. The abscissa represents the mass cumulatively lost through stellar winds, expressed as a fraction of the initial mass. Also shown is the $\mathrm{C} / \mathrm{O}$ ratio. The vertical line indicates when $\mathrm{C} / \mathrm{O}=1$. In the subsequent evolution (to the right of the line) the star is carbon-rich, i.e. when a $\mathrm{C}$ star develops.

susceptible to any possible changes due to the well-defined production rates. In addition, we note that ${ }^{107} \mathrm{Pd}$ is abundant in the UP model. The high abundance of ${ }^{107} \mathrm{Pd}$ in the UP model is diminished by decay (over $\sim 10^{8}$ years) after the multiple $r$-process source proposed by Wasserburg et al (1996). As a result, ${ }^{107} \mathrm{Pd}$ from the ISM was taken to be negligible at the time of solar system formation. If, however, some of the ${ }^{107} \mathrm{Pd}$ in the ESS came from UP, then this would require an even lower production of ${ }^{107} \mathrm{Pd}$ from an $\mathrm{AGB}$ source. It therefore follows that, if the source 


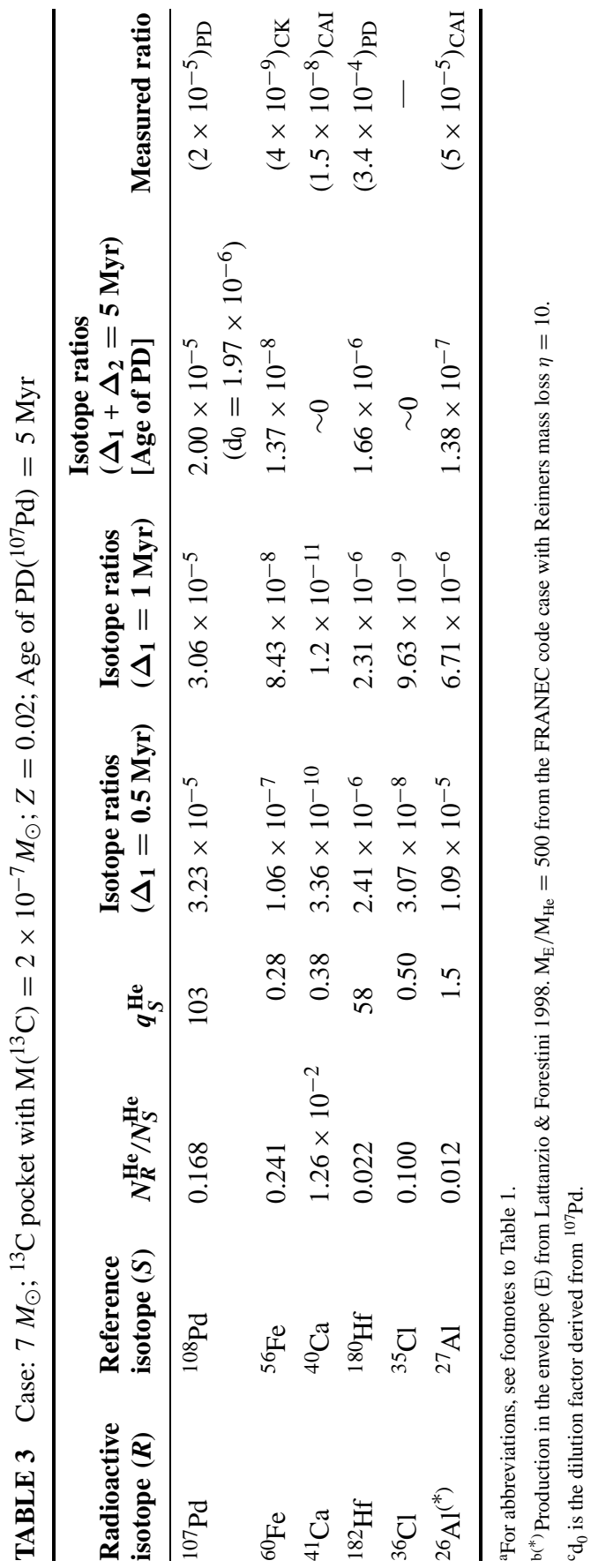


of late addition to the ESS comes from an AGB star, it must have been dominated by neutrons from the ${ }^{22} \mathrm{Ne}$ source in the convective He shell alone and have only a very small to negligible contribution from any ${ }^{13} \mathrm{C}$ pocket. The question of a fully quantitative agreement cannot yet be answered.

As for more detailed characteristics of a possible AGB source, we thus consider that any model source that grossly (within an order of magnitude) underproduces any of the nuclei ${ }^{41} \mathrm{Ca},{ }^{26} \mathrm{Al}$, and ${ }^{60} \mathrm{Fe}$ must be excluded. The same is true if the model requires a time scale (as determined by ${ }^{41} \mathrm{Ca}$ ) shorter than a reasonable free-fall time $\left(\sim 3 \times 10^{5}\right.$ years, see Wasserburg et al 1995). The overproduction of a nuclide such as ${ }^{60} \mathrm{Fe}$ can, in principle, be accounted for by changing $\Delta_{1}+\Delta_{2}$. The most serious question then is regarding the production of ${ }^{26} \mathrm{Al}$. As discussed earlier, our assessment of the ${ }^{26} \mathrm{Al}$ yields has uncertainties caused both by the production/destruction and the details of transport. Insofar as the TDU is properly described in the present models, we are thus left with the issue of the reaction rates, especially for the ${ }^{26} \mathrm{Al}(\mathrm{p}, \gamma)$ reaction.

An IMS with hot bottom burning appears to be excluded because of low ${ }^{41} \mathrm{Ca}$. The remaining choices are LMS between 1.5 and $3 M_{\odot}$. The $1.5-M_{\odot}$ case has a value for ${ }^{26} \mathrm{Al}$ that is too low by a factor of $\sim 3$, and the $3-M_{\odot}$ case is too low by a factor of 8 . This matter can be resolved only if there is a significant decrease in the ${ }^{26} \mathrm{Al}$ destruction rate by proton capture as must be determined experimentally, or if there is an additional mechanism that may produce ${ }^{26} \mathrm{Al}$ without major destruction (e.g. CBP). The production of ${ }^{26} \mathrm{Al}$ has been found to be problematical for stars of $3 M_{\odot}$. However, if the production were found to be more efficient than currently calculated values or if $\mathrm{CBP}$ were effective in producing ${ }^{26} \mathrm{Al}$, then another polluting source may also be an AGB star of perhaps $3 M_{\odot}$ within the cloud. This would enable a self-contaminating mechanism within the cloud without requiring an encounter.

The ${ }^{60} \mathrm{Fe}$ remains an issue owing to the matter of early solar system time scales $\left(\Delta_{1}+\Delta_{2}\right)$. A reliable determination must be made of ${ }^{60} \mathrm{Fe} /{ }^{56} \mathrm{Fe}$ on samples with a known ${ }^{26} \mathrm{Al} /{ }^{27} \mathrm{Al}$ (Wasserburg et al 1998). This would eliminate the floating parameter $\left(\Delta_{2}\right)$ and come close to establishing a quantitative scale for assessing the legitimacy of an AGB source. The experimental works on ${ }^{60} \mathrm{Fe}$ by Shukolyukov \& Lugmair (1993a,b) are of great importance. However, we note that the available data on ${ }^{60} \mathrm{Fe} /{ }^{56} \mathrm{Fe}$ is very limited, and the experimental difficulties of measuring and correlating ${ }^{60} \mathrm{Ni}$ excesses with $\mathrm{Fe}$ are formidable. Further, there are at present no data on ${ }^{60} \mathrm{Fe}$ in CAIs in which ${ }^{26} \mathrm{Al}$ was present or in the rare chondrules with some small levels of ${ }^{26} \mathrm{Al}$. In addition, although ${ }^{53} \mathrm{Mn} /{ }^{55} \mathrm{Mn}$ in planetary differentiates is now very well established, there has been no serious effort to determine this on samples showing the presence of ${ }^{26} \mathrm{Al}$ although the original work by Birck \& Allègre $(1985,1988)$ on CAIs suggests a connection. A quantitative relationship between ${ }^{53} \mathrm{Mn}$ and ${ }^{26} \mathrm{Al}$ for CAIs would provide a clear estimate of the ${ }^{53} \mathrm{Mn}$ inventory of the ESS that has been postulated to be from the ISM as a product of longer-term UP (Wasserburg et al 1996). These data on ${ }^{53} \mathrm{Mn}$ and ${ }^{26} \mathrm{Al}$ would also be used to eliminate the uncertainty in $\Delta_{1}+\Delta_{2}$, using the connection through 
${ }^{53} \mathrm{Mn}$ in both CAIs and planetary differentiates. There is always the problem of multistage metamorphism (e.g. see Figure 18d,e) so that a simple assignment of $\Delta_{2}$ to an object is not a priori evident.

Can Contamination Happen? We now try to address the plausibility that an AGB star with suitable characteristics could encounter a protosolar cloud at the right distance to pollute it with short-lived nuclei. It was argued by Kastner \& Myers (1994) that today the probability of a close encounter between a masslosing AGB star and a molecular cloud in the solar neighborhood is one encounter in $10^{6}$ years. In the scenario emphasized in the present section, the AGB star having suitable nucleosynthesis yields is of low mass $\left(\sim 1.5 M_{\odot}\right)$ and is therefore certainly older than the molecular cloud out of which the sun may have formed. A chance encounter is therefore necessary. We underline, however, a series of issues that have to be considered. As noted by Kastner \& Myers (1994), the number of AGB stars at the time of the solar system formation was probably much larger than today (perhaps a factor $e^{2}$; see Wyse \& Silk 1987). Among these AGBs, a low-mass star would certainly be favored in the initial mass function as compared with an IMS. Moreover, while cloud cores of large mass, forming clusters, are associated with high-density molecular regions (Myers 1998), low-mass cores like the one from which the sun plausibly formed are often found in relatively lowdensity molecular clouds [e.g. in Taurus (Codella et al 1997)]. This increases the probability of encounter consistently and may possibly not require the pollution of a large cloud mass, but only of the cloud core itself.

Basic dynamical questions to be answered are (a) can the shock wave from the blown-off envelope provide the required momentum to trigger the collapse of a dense cloud core on a short time scale without disrupting it; and $(b)$ can there be entrapment $(\sim 1 \%)$ of the ejected material in the collapsing SC? These problems also pertain to the suggestion of a supernova trigger (Cameron \& Truran 1977) and have received recent attention in a number of works (Boss 1995, Foster \& Boss 1998, Boss \& Foster 1998, Vanhala \& Cameron 1998). It was found that, for shock velocities of 10-25 km/s, the collapse of the dense core can be obtained, with an $\sim 1 \%$ entrapment of fresh debris. It appears that this may be a plausible mechanism for both triggering collapse and injecting freshly synthesized matter. However, the dynamical problem is complex and will undoubtedly be the object of intense study in the near future.

\section{CONCLUSIONS}

In recent years there has been significant progress in studies of the late stages of evolution of low and intermediate mass stars. In some stellar evolutionary codes, using computational schemes at high resolution and improved opacities and requiring only the Schwarzschild criterion for convection, third dredge-up is found to self-consistently occur in AGB stars of low mass and nearly solar metallicity. 
It remains to be seen whether this approach will provide the generally accepted solution to the third dredge-up problem. These new models allow the formation of low-mass carbon stars at the appropriate luminosities in accordance with observations. It was further found that the ${ }^{13} \mathrm{C}(\alpha, n){ }^{16} \mathrm{O}$ reaction takes place under radiative conditions (not convective) during the interpulse periods in a layer below the H-burning zone. It is in this region that the dominant $s$ processing takes place in AGB stars at a relatively low neutron density $\left(\sim 10^{7} \mathrm{n} \mathrm{cm}^{-3}\right)$. Subsequent entrainment of this $s$-processed material into the thermal pulses exposes the mixed matter to a weak neutron source from the ${ }^{22} \mathrm{Ne}(\alpha, \mathrm{n})^{25} \mathrm{Mg}$ reaction at somewhat higher neutron densities $\left(\lesssim 5 \times 10^{10} \mathrm{n} \mathrm{cm}^{-3}\right)$ than were previously obtained. With these recent stellar models, full $s$-process calculations have been done for both LMS and IMS. Based on the burning of ${ }^{13} \mathrm{C}$ under radiative conditions, the distribution of neutron exposures during the $s$ process cannot be approximated by an exponential form, as is instead assumed in the phenomenological approach (and was achieved in stellar models in which the ${ }^{13} \mathrm{C}$ neutron source was also assumed to operate within the convective pulse). It appears that the solar system $s$-process abundances are not the result of a unique $s$ process, but rather of galactic chemical evolution, which mixes the products of $s$ processing in stars of different metallicity and with a range of ${ }^{13} \mathrm{C}$ pockets. The mechanism of formation of the ${ }^{13} \mathrm{C}$ pocket still remains a fundamental problem. As such, it is selected to fit the observational data. Current models show that the third dredge-up establishes a sharp discontinuity between the $\mathrm{H}$-rich and ${ }^{12} \mathrm{C}$-rich layers. This suggests that the ${ }^{13} \mathrm{C}$ pocket is the result of transport mechanisms that mix protons from the convective envelope into the radiative ${ }^{12} \mathrm{C}$-rich zone, where they are subsequently burned to ${ }^{13} \mathrm{C}$. Dedicated hydrodynamical studies of this boundary region are required to establish the actual mechanism of formation of the ${ }^{13} \mathrm{C}$ pocket. Until this is accomplished and shown to be a natural consequence of stellar evolution models, the ${ }^{13} \mathrm{C}$ pocket remains a matter of parameterization in $s$-process nucleosynthesis.

There is also the problem of additional mixing of the envelope down to regions close to the H-burning shell. This is required by the observational data on LMS for $\mathrm{C}$ and $\mathrm{O}$ isotopes, and on the precise measurements of $\mathrm{O}$ on circumstellar dust grains extracted from meteorites. While slow convective mixing down to just above the H-burning shell [cool bottom processing (CBP)] is required by the observations, it does not appear as a natural consequence of the existing models of stellar evolution and may again require a very detailed hydrodynamical treatment. We also note that all of the stellar calculations discussed here are based on onedimensional models and that three-dimensional dynamics may define transport mechanisms that are obscured by the one-dimensional treatment. This might be the cause of some level of transport near the $\mathrm{H}$ shell that is required by CBP. Threedimensional hydrodynamics may involve the role of convective plumes, which could affect both the above mixing problems and also the transport of "delicate" nuclei such as Li to the stellar envelope. We further note that the problem of mass loss has so far also been treated parametrically; dynamical models are still in 
their infancy, particularly as related to high-mass-loss regimes and to sporadicmass-loss enhancements. We recognize that there are great difficulties in treating the external boundary of the star, where there are complex interactions between neutral gas, dust, charged particles, radiation, and the associated dynamics of loss.

Insofar as the stellar evolution is now properly described by the major nuclearreaction rates, the opacity function, and the mixing-length parameter $\alpha_{p}, s$-process enhancements in stellar envelopes in the AGB models show that the envelope compositions are functions of the initial mass, mass loss rate, metallicity, and ${ }^{13} \mathrm{C}$ pocket. For a given initial stellar mass and metallicity, the composition is then dependent on two arbitrary parameters, the mass of the ${ }^{13} \mathrm{C}$ pocket and the mass loss rate. Current models appear to provide a good quantitative description of several observations and also a basis for estimating the reasons for the abundance scatter that is evident at any metallicity. There are many remaining problems, particularly concerning very low-metallicity stars $([\mathrm{Fe} / \mathrm{H}]<-2)$ and the Li-rich stars.

Whereas many radioactive isotopes in the ESS with intermediate lifetimes $\left({ }^{244} \mathrm{Pu},{ }^{247} \mathrm{Cm},{ }^{182} \mathrm{Hf},{ }^{129} \mathrm{I}\right.$, and $\left.{ }^{53} \mathrm{Mn}\right)$ appear to derive from the standing inventory in the ISM provided by diverse supernova sources, the abundances of some short-lived nuclei $\left({ }^{26} \mathrm{Al},{ }^{41} \mathrm{Ca},{ }^{60} \mathrm{Fe}\right.$, and $\left.{ }^{107} \mathrm{Pd}\right)$ are compatible with a contamination of the placental nebular cloud from which the sun formed with the ejecta of a closeby AGB source. The new AGB models show that the source must be a low-mass star $\left(\sim 1.5 M_{\odot}\right)$. Higher-mass stars appear to be excluded because of inadequate ${ }^{26} \mathrm{Al}$ and/or ${ }^{41} \mathrm{Ca}$ production. There appears to be some problem with the extent to which AGB stars can provide sufficient ${ }^{26} \mathrm{Al}$. There is a marginal shortfall of ${ }^{26} \mathrm{Al}$ production in a $1.5-M_{\odot}$ star and, for higher-mass stars, a substantial shortfall. The results on ${ }^{26} \mathrm{Al}$ are critically dependent on some nuclear-reaction rates that need to be established and on the details of transport at the third dredge-up, because the destruction of ${ }^{26} \mathrm{Al}$ in the thermal pulses controls the amount in the envelope. It is possible that $\mathrm{CBP}$ contributes to ${ }^{26} \mathrm{Al}$ production in the envelope and should be investigated. The question of a supernova or AGB star as a source of short-lived contaminants and as a possible trigger remains to be tested. A possible test may be the ${ }^{26} \mathrm{Al} /{ }^{60} \mathrm{Fe}$ ratio in the early solar system (Wasserburg et al 1998).

\section{ACKNOWLEDGMENTS}

We acknowledge valuable comments by A McWilliam concerning the manuscript and Allan Sandage's critical and constructive input. We thank O Straniero and A Chieffi for useful suggestions. We are deeply indebted to ME Johnson for her continuous help in the preparation of $6.023 \times 10^{23}$ successive versions of this work. MB and RG are grateful to the Lunatic Asylum and to Caltech for their hospitality. This work was supported by NASA grants NAG5-4076 and NAG54083, and by a MURST Cofin98 Italian grant. Caltech Division Contribution 8594(1012). 


\section{Visit the Annual Reviews home page at http://www.AnnualReviews.org}

\section{LITERATURE CITED}

Alexander DR, Ferguson JW. 1994. Ap. J. 437:879

Alexander EC Jr, Lewis RS, Reynolds JH, Michel MC. 1971. Science 172:837

Alongi M, Bertelli G, Bressan A, Chiosi C. 1991. Astron. Astrophys. 244:95

Anders E, Grevesse N. 1989. Geochim. Cosmochim. Acta 53:197

Anders E, Zinner E. 1993. Meteoritics 28: 490

Anders E, Zinner E. 1994. Icarus 112:303

Aoki W, Tsuji T, Ohnaka K. 1998a. Astron. Astrophys. 333:L19

Aoki W, Tsuji T, Ohnaka K. 1998b. Astron. Astrophys. 340:222

Arndt TU, Fleischer AJ, Sedlmayr E. 1997. Astron. Astrophys. 327:614

Arnould M, Mowlavi N, Champagne AE. 1995. In Stellar Evolution. What Should Be Done. XXXII Liège Astrophys. Colloq. ed. A Noels, D Fraipont-Caro, M Gabriel, N Grevesse, B Demarque, p. 17. Liège, Belgium: Inst. d'Astrophys.

Bahcall J, Pinsonneault MH, Wasserburg GJ. 1995. Rev. Mod. Phys. 67:781

Bao ZY, Käppeler F. 1987. At. Data Nucl. Data Tables 36:411

Barnes CA. 1982. In Essays in Nuclear Astrophysics, ed. CA Barnes, DD Clayton, DN Schramm, p. 193. Cambridge, U.K.: Cambridge Univ. Press

Bazan G, Lattanzio JC. 1993. Ap. J. 409:726

Becker SA. 1981. In Physical Processes in Red Giants, ed. I Iben Jr, A Renzini, p. 121. Dordrecht, The Netherlands: Reidel

Becker SA, Iben I Jr. 1980. Ap. J. 232:831

Beer H, Voss F, Winters RR. 1992. Ap. J. Suppl. 80:403

Bernatowicz TJ, Amari S, Zinner EK, Lewis RS. 1991. Ap. J. 373:L73

Bernatowicz TJ, Cowsik R, Gibbons PC, Lodders K, Fegley B, et al. 1996. Ap. J. 472: 760
Bertelli G, Bressan A, Chiosi C. 1985. Ap. J. 392:522

Birck J-L, Allègre CA. 1985. Geophys. Res. Lett. 12:745

Birck J-L, Allègre CA. 1988. Nature 331:579

Blanco VM, McCarthy M, Blanco BM. 1980. Ap. J. 242:938

Blöcker T. 1995a. Astron. Astrophys. 299:755

Blöcker T. 1995b. Astron. Astrophys. 297:727

Blöcker T, Schönberner D. 1991. Astron. Astrophys. 244:L43

Blöcker T, Schönberner D. 1996. Mem. Soc. Sci. Liège 32:455

Blommaert JADJ, Trams N, Okumura K, Groenewegen MAT, Cioni MR, et al. 1998. Astrophys. Space Sci. 255:399

Boothroyd AI, Sackmann I-J. 1988a. Ap. J. 328:632

Boothroyd AI, Sackmann I-J. 1988b. Ap. J. 328:641

Boothroyd AI, Sackmann I-J. 1988c. Ap. J. 328:653

Boothroyd AI, Sackmann I-J. 1988d. Ap. J. 328:672

Boothroyd AI, Sackmann I-J, Wasserburg GJ. 1994. Ap. J. 430:L77

Boothroyd AI, Sackmann I-J, Wasserburg GJ. 1995. Ap. J. 442:L21

Boss AP. 1995. Ap. J. 439:224

Boss AP, Foster PN. 1997. In Proc. AIP Conf. 402, Astrophysical Implications Laboratory Study Presolar Materials, St. Louis, p. 649. Woodbury, NY: Am. Inst. Phys.

Boss AP, Foster PN. 1998. Ap. J. 494:L103

Brazzle RH, Pravdivtseva OV, Meshik AP, Hohenberg CM. 1999. Geochim. Cosmochim. Acta. 63. In press

Bujarrabal V, Fuente A, Omont A. 1994a. Astron. Astrophys. 285:247

Bujarrabal V, Fuente A, Omont A. 1994b. Ap. J. 421:L47

Burbidge EM, Burbidge GR, Fowler WA, Hoyle F. 1957. Rev. Mod. Phys. 29:547 
Busso M, Gallino R. 1997. Nucl. Phys. A 621: C431

Busso M, Gallino R, Raiteri CM, eds. 1995a. Proc. AIP Conf. Nuclei in the Cosmos, III, Assergi, Italy. 327, p. 625. Woodbury, NY: Am. Inst. Phys.

Busso M, Lambert DL, Beglio L, Gallino R. 1995b. Ap. J. 446:775

Busso M, Picchio G, Gallino R, Chieffi A. 1988. Ap. J. 326:196

Buzzoni A, Fusi Pecci F, Buonanno R, Corsi CE. 1983. Astron. Astrophys. 128:94

Cameron AGW. 1954. Phys. Rep. 93:932

Cameron AGW. 1957. Chalk River Rep. CRL41

Cameron AGW. 1960. A. J. 65:485

Cameron AGW. 1993. In Protostars and Planets III, ed. EH Levy, JL Lunine, p. 47. Tucson: Univ. Arizona Press

Cameron AGW, Thielemann FK, Cowan JJ. 1993. Phys. Rep. 227:283

Cameron AGW, Truran JW. 1977. Icarus 30: 447

Cameron AGW, Vanhala H, Höflich P. 1997. In Proc. AIP Conf. 402, Astrophysical Implications Laboratory Study Presolar Materials St. Louis, ed. TJ Bernatowicz, E Zinner, p. 667. Woodbury, NY: Am. Inst. Phys.

Castellani V, Giannone P, Renzini A. 1969. Astrophys. Space Sci. 4:103

Caughlan GR, Fowler WA, Harris MJ, Zimmerman BA. 1985. At. Data Nucl. Data Tables 32:197

Caughlan GR, Fowler WA. 1988. At. Data. Nucl. Data Tables 40:283

Champagne AE, Wiescher M. 1992. Annu. Rev. Nucl. Part. Sci. 42:39

Chan SJ, Kwok S. 1988. Ap. J. 334:362

Charbonnel C. 1994. Astron. Astrophys. 282: 811

Charbonnel C. 1995. Ap. J. 453:L41

Chen JH, Wasserburg GJ. 1996. In Earth Processes: Reading the Isotopic Code, ed. A. Basu, S Hart. Geophys. Monogr. 95:1. Washington, DC: Am. Geophys. Union

Chen JH, Wasserburg GJ. 1981a. Earth Planet. Sci. Lett. 52:1
Chen JH, Wasserburg GJ. 1981b. Anal. Chem. 53:2060

Chiosi C, Bertelli G, Bressan A. 1992. Annu. Rev. Astron. Astrophys. 30:235

Choi B-G, Huss GR, Wasserburg GJ, Gallino R. 1998. Science 282:1284

Clayton DD. 1968. Principles of Stellar Evolution and Nucleosynthesis. Chicago: Univ. Chicago Press. 612 pp.

Clayton DD. 1988. MNRAS 234:1

Clayton DD, Fowler WA, Hull TE, Zimmerman BA. 1961. Ann. Phys. 12:331

Clayton DD, Jin LP. 1995. Ap. J. 451:681

Clayton DD, Ward RA. 1974. Ap. J. 193:397

Clegg RES, Lambert DL, Bell RA. 1979. Ap. J. 234:188

Codella C, Welser R, Henkel C, Benson PJ, Myers PC. 1997. Astron. Astrophys. 324:203

Cohen M. 1984. MNRAS 206:137

Cosner K, Iben I Jr, Truran JW. 1980. Ap. J. 238:L91

Cowan JJ, Pfeiffer B, Kratz K-L, Thielemann F-K, Sneden C, et al. 1999. Ap. J. In press

Cowan JJ, Sneden C, Truran JW, Burris DL. 1996. Ap. J. 460:L115

Crawford JL, Sneden C, King JR, Boesgaard AM, Deliyannis CP. 1998. A. J. 116:2489

D’Antona F, Mazzittelli I. 1996. Ap. J. 470: 1093

Dearborn DSP. 1992. Phys. Rep. 210:367

Decin L, Van Winckel H, Waelkens C, Bakker EJ. 1998. Astron. Astrophys. 332:929

Denissenkov PA, Weiss A. 1996. Astron. Astrophys. 308:773

Denker A, Drotleff HW, Grosse M, Knee H, Kunz R, et al. 1995. In Proc. AIP Conf. 327, Nuclei Cosmos III Assergi Italy, ed. M Busso, CM Raiteri, R Gallino, p. 255. New York: Am. Inst. Phys.

Despain KH. 1980. Ap. J. 236:648

Diehl R. Timmes FX. 1998. PASP 110:637

Dominy JF, Wallerstein G. 1986. Ap. J. 310: 371

Edvardsson B, Andersen J, Gustafsson B, Lambert DL, Nissen PE, Tomkin J. 1993. Astron. Astrophys. 275:101

El Eid MF. 1994. Astron. Astrophys. 285:915 
Ferrini F, Matteucci F, Pardi C, Penco U. 1992. Ap. J. $387: 138$

Forestini M, Charbonnel M. 1997. Astron. Astrophys. Suppl. 123:241

Forestini M, Paulus G, Arnould M. 1991. Astron. Astrophys. 252:597

Foster PN, Boss AP. 1998. Ap. J. 494:L103

Fowler WA, Caughlan GR, Zimmermann BA. 1967. Annu. Rev. Astron. Astrophys. 5: 525

Fowler WA, Caughlan GR, Zimmermann BA. 1975. Annu. Rev. Astron. Astrophys. 13:69

Fowler WA, Hoyle F. 1960. Ann. Phys. 10: 280

Frogel JA, Mould J, Blanco VM. 1990. Ap. J. 352:96

Frost CA, Lattanzio JC. 1996. Ap. J. 473:383

Frost CA, Lattanzio JC, Wood PR. 1998. Ap. J. 500:355

Fuller GM, Fowler WA, Newman MJ. 1985. Ap. J. 293:1

Gallino R. 1989. In Evolution of Peculiar Red Giant Stars, ed. HR Johnson, B Zuckerman, International Astronomical Union (IAU) Collog. 106:176. Cambridge, U.K.: Cambridge Univ. Press

Gallino R, Arlandini C, Busso M, Lugaro M, Travaglio C, et al. 1998. Ap. J. 497:388

Gallino R, Busso M, Lugaro M. 1997. In Proc. AIP Conf. 402, Astrophysical Implications Laboratory Study Presolar Materials, ed. TJ Bernatowicz, E Zinner, St. Louis Mo, p. 115. Woodbury, NY: Am. Inst. Phys.

Gallino R, Busso M, Lugaro M, Travaglio C, Vaglio P. 1999. In Nuclei in the Cosmos V, ed. N Prantzos, S Harissopulos, p. 216. Paris: Ed. Frontières.

Gallino R, Busso M, Picchio G, Raiteri CM, Renzini A. 1988. Ap. J. 334:L45

Gallino R, Raiteri CM, Busso M. 1993. Ap. J. 410:400

García-Berro E, Ritossa C, Iben I Jr. 1997. Ap. J. $485: 765$

Gilroy KK. 1989. Ap. J. 347:835

Gilroy KK, Brown JA. 1991. Ap. J. 371:578

Gilroy KK, Sneden C, Pilachowski CA, Cowan JJ. 1988. Ap. J. 327:298
Glassgold AE. 1996. Annu. Rev. Astron. Astrophys. 34:241

Glassgold AE, Feigelson ED, Montmerle T. 1998. In Protostars \& Planets. Tucson: Univ. Arizona Press. In press

González G, Wallerstein G. 1992. MNRAS 254: 343

Görres J, Mathews G, Wiescher M, Shore S, eds. 1997. Nuclei in the Cosmos IV, Nucl. Phys. A 621:1c-643c

Gratton RG. 1996. In AGB Evolution and Nucleosynthesis, ed. M Busso, R Gallino, C Arlandini, Mem. Soc. Astron. It. 67:777

Gratton RG, Ortolani S. 1986. Astron. Astrophys. 169:201

Gratton RG, Sneden C. 1994. Astron. Astrophys. 287:927

Greenstein JL. 1954. In Modern Physics for Engineers, ed. L Ridenour. New York: McGraw-Hill

Grevesse N, Noels A, Sauval AJ. 1996. In Cosmic Abundances, ed. SS Holt, G Sonneborn. ASP Conf. Ser. Vol. 99, p. 117. San Francisco: Astronomical Society of the Pacific

Groenewegen MAT, de Jong T. 1999. Astron. Astrophys. In press

Gustafsson B. 1989. Annu. Rev. Astron. Astrophys. 27:701

Harper CL, Jacobsen SB. 1994. Lunar Planet. Sci. 25:509

Harper CL, Jacobsen SB. 1996. Geochim. Cosmochim. Acta 60:1131

Hartwick FDA, Härm R, Schwarzschild M. 1968. Ap. J. 153:389

Herwig F, Blöcker T, Schönberner D, El Eid M. 1997. Astron. Astrophys. 324:L81

Herwig F, Schönberner D, Blöcker T. 1998. Astron. Astrophys. 340:L43

Hollowell D, Iben I Jr. 1988. Ap. J. 333:L25

Hollowell D, Iben I Jr. 1989. Ap. J. 340:966

Hollowell D, Iben I Jr. 1990. Ap. J. 349:208

Holmes JA, Woosley WA, Fowler WA, Zimmerman BA. 1976. At. Data Nucl. Data Tables 18:305

Hoppe P, Ott U. 1997. In Proc. AIP Conf. 402, Astrophysical Implications Laboratory Study Presolar Materials, St. Louis Mo, ed. TJ 
Bernatowicz, EZinner, p. 27. Woodbury, NY: Am. Inst. Phys.

Hoyle F, Schwarzschild M. 1955. Ap. J. Suppl. 2:1

Howard WM, Mathews GJ, Takahashi K, Ward RA. 1986. Ap. J. 309:633

Hron J, Loidl R, Hofner S, Jorgensen UG, Aringer B, Kerschbaum F. 1997. Astron. Astrophys. 335:L69

Hudson GB, Kennedy BM, Podosek FA, Hohenberg CM. 1989. Proc. Lunar Planet. Sci. Conf. 19:547

Huss GR, Fahey AJ, Gallino R, Wasserburg GJ. 1994. Ap. J. 430:L81

Huss GR, Lewis RS. 1995. Geochim. Cosmochim. Acta 59:115

Hutcheon ID, Krot AN, Keil K, Phinney DL, Scott ERD. 1998. Science 282:1865

Iben I Jr. 1973. Ap. J. 185:209

Iben I Jr. 1975. Ap. J. 196:525

Iben I Jr. 1976. Ap. J. 208:165

Iben I Jr. 1977. In Advanced Stages of Stellar Evolution, ed. P Bouvier, A Maeder, p. 1. Sauverny, Switzerland: Geneva Obs.

Iben I Jr. 1982. Ap. J. 260:821

Iben I Jr. 1983. Ap. J. 275:L65

Iben I Jr. 1984. Ap. J. 277:333

Iben I Jr. 1991. In Evolution of Stars: the Photospheric Abundance Connection, ed. G Michaud, A Tutukov. IAU Symp. 145:257. Dordrecht, The Netherlands: Kluwer

Iben I Jr, Renzini A. 1982a. Ap. J. 249:L79

Iben I Jr, Renzini A. 1982b. Ap. J. 263:L23

Iben I Jr, Renzini A. 1983. Annu. Rev. Astron. Astrophys. 21:271

Iben I Jr, Renzini A. 1984. Phys. Rep. 105: 329

Iben I Jr, Ritossa C, García-Berro E. 1997. Ap. J. 489:772

Iben I Jr, Truran JW. 1978. Ap. J. 220:980

Israelian G, García López RJ, Rebolo R. 1998. Ap. J. 507:805

Izumiura H, Hashimoto O, Kawara K, Yamamura I, Waters LBFM. 1996. Astron. Astrophys. 315:L221

Jacobsen SB, Harper CL. 1996. In Earth Processes: Reading the Isotopic Code, ed.
A Basu, S Hart. Geophys. Monogr. 95:47. Washington, DC: Am. Geophys. Union

Jacobsen SB, Wasserburg GJ. 1984. Earth Planet. Sci. Lett. 67:137

Jeffrey PM, Reynolds JH. 1961. J. Geophys. Res. 66:3582

Jorissen A, Mayor M. 1992. Astron. Astrophys. 260:115

Jura M. 1997. In Proc. AIP Conf. 402, Astrophysical Implications Laboratory Study Presolar Materials, ed. TJ Bernatowicz, E Zinner, p. 379. Woodbury, NY: Am. Inst. Phys.

Jura M, Kahane C, Omont A. 1988. Astron. Astrophys. 201:80

Jura M, Kleinmann SG. 1989. Ap. J. 341:359

Kahane C, Cernicharo J, Gómez-González J, Guélin M. 1992. Astron. Astrophys. 256: 235

Käppeler F. 1997. In Nuclei in the Cosmos IV, ed. J Görres, G Mathews, M Wiescher, S Shore. Nucl. Phys. A 621:221c

Käppeler F. 1999. In Nuclei in the Cosmos V, ed. N Prantzos, S Harissopulos, p. 174. Paris: Ed. Frontières.

Käppeler F, Beer H, Wisshak K. 1989. Rep. Prog. Phys. 52:945

Käppeler F, Beer H, Wisshak K, Clayton DD, Macklin RL, Ward RA. 1982. Ap. J. 257:821

Käppeler F, Gallino R, Busso M, Picchio G, Raiteri CM. 1990. Ap. J. 354:630

Käppeler F, Wiescher M, Giesen U, Görres J, Baraffe I, et al. 1994. Ap. J. 437:396

Käppeler F, Wisshak K, eds. 1993. Nuclei in the Cosmos II, Bristol, U.K.: Inst. Phys.

Kastner JH, Myers PC. 1994. Ap. J. 421:605

Kavanagh RW. 1982. In Essays in Nuclear Astrophysics, ed. CA Barnes, DD Clayton, DN Schramm, p. 159. Cambridge, U.K.: Cambridge Univ. Press

Kelly WR, Wasserburg GJ. 1978. Geophys. Res. Lett. 5:1079

Kilston S. 1985. PASP 97:189

Kipper T. 1991. In Evolution of Stars: the Photospheric Abundance Connection, ed. G Michaud, A Tutukov, IAU Symp. 145:317. Dordrecht, The Netherlands: Kluwer 
Knapp GR, Chang KM. 1985. Ap. J. 293:281

Kraft RP. 1994. PASP 106:553

Kraft RP, Sneden C, Langer GE, Shetrone MD. 1993. Astron. J. 106:1490

Kurucz RL. 1991. In Stellar Atmospheres: Beyond Classical Models, ed. L Crivellari, I Hubeny, DG Hummer, p. 141. Dordrecht, The Netherlands: Kluwer

Lambert DL. 1985. In Cool Stars with Excesses of Heavy Elements, ed. M Jaschek, PC Keenan, p. 191. Dordrecht, The Netherlands: Reidel

Lambert DL, Ries LM. 1981. Ap. J. 248:228

Lambert DL, Smith VV, Busso M, Gallino R, Straniero O. 1995. Ap. J. 450:302

Langer N. 1986. Astron. Astrophys. 164:45

Langer N, Heger A, Woosley SE, Herwig F. 1999. In Nuclei in the Cosmos V, ed. $\mathrm{N}$ Prantzos. S Harissopulos, p. 129. Paris: Ed. Frontières

LaTourrette T, Wasserburg GJ. 1998. Earth Planet. Sci. Lett. 158:91

Lattanzio JC. 1986. Ap. J. 311:708

Lattanzio JC. 1989. Ap. J. 344:L25

Lattanzio JC. 1995. In Nuclei in the Cosmos III, ed. M Busso, R Gallino, CM Raiteri, AIP Conf. Proc. 327:591. Woodbury, New York: AIP

Lattanzio JC, Boothroyd A. 1997. In Proc. AIP Conf. 402, Astrophysical Implications Laboratory Study Presolar Materials, ed. TJ Bernatowicz, E Zinner, St. Louis Mo, p. 85. Woodbury, NY: Am. Inst. Phys.

Lattanzio JC, Frost C, Cannon R, Wood P. 1996. In AGB Evolution and Nucleosynthesis, ed. M Busso, R Gallino, C Arlandini, Mem. Soc. Astron. It. 67:729

Le Bertre T. 1998. Astron. Astrophys. 203:85

Lee D-C, Halliday AN. 1995. Nature 378:771

Lee D-C, Halliday AN. 1996. Science 274:1876

Lee D-C, Halliday AN. 1997. Nature 388:854

Lee D-C, Halliday AN. 1998. Lunar Planet. Sci. 29:1416

Lee D-C, Halliday AN. 1999. Chem. Geol. In press

Lee T, Papanastassiou DA, Wasserburg GJ. 1977. Ap. J. 211:L107
Lee T, Papanastassiou DA, Wasserburg GJ. Geophys. Res. Lett. 3:109

Lee T, Shu FH, Shang H, Glassgold AE, Rehm KE. 1998. Ap. J. 506:898

Little SJ, Little-Marenin IR, Hagen Bauer W. 1987. Ap. J. 94:981

Little-Marenin IR. 1986. Ap. J. 307:L15

Little-Marenin IR. 1989. In Evolution of Peculiar Red Giant Stars, ed. HR Johnson, B Zuckerman, IAU Coll. 106:131. Cambridge, U.K.: Cambridge Univ. Press

Lodders K, Fegley B Jr. 1995. Meteoritics 30: 661

Lodders K, Fegley B Jr. 1997. In Proc. AIP Conf. 402, Astrophysical Implications Laboratory Study Presolar Materials, ed. TJ Bernatowicz, E Zinner, p. 391. Woodbury, NY: Am. Inst. Phys.

Loup C, Forveille T, Omont A, Paul JF. 1993. Astron. Astrophys. Suppl. 99:291

Luck RE, Bond HE. 1981. PASP 93:211

Luck RE, Bond HE. 1984. Ap. J. 279:729

Luck RE, Bond HE. 1985. Ap. J. 292:559

Luck RE, Bond HE. 1991. Ap. J. Suppl. 77:515

Luck RE, Bond HE, Lambert DL. 1990. Ap. J. 357:188

Luck RE, Lambert DL. 1982. Ap. J. 256:189

Lugmair GW, Marti K. 1977. Earth Planet. Sci. Lett. 35:273

Lugmair GW, Shukolyukov A. 1998. Geochim. Cosmochim. Acta 62:2863

MacPherson GJ, Davis AM, Zinner EK. 1995. Meteoritics 30:365

Maeder A. 1975. Astron. Astrophys. 40:303

Malaney RA. 1986. MNRAS 223:683

Malaney RA. 1987. Astrophys. Space Sci. 137: 251

Marigo P, Bressan A, Chiosi C. 1996. Astron. Astrophys. 313:545

Martin PG, Rogers C. 1987. Ap. J. 322:374

Mathews GJ, Bazan G, Cowan JJ. 1992. Ap. J. 391:719

Mathews GJ, Takahashi K, Ward RA. 1986. Ap. J. 302:410

Mathews GJ, Ward RA. 1985. Rep. Prog. Phys. 48:1371

McClure RD. 1984. PASP 96:117 
McClure RD, Fletcher JM, Nemec JM. 1980. Ap. J. 238:L35

McCulloch MT, Wasserburg GJ. 1978. Ap. J. 220:L15

McWilliam A. 1995. Annu. Rev. Astron. Astrophys. 35:503

McWilliam A. 1998. Ap. J. 115:1640

McWilliam A, Lambert DL. 1988. MNRAS 230: 573

McWilliam A, Preston GW, Sneden C, Searle L. 1995. Astron. J. 109:2757

McWilliam A, Searle L. 1999. In Galaxy Evolution: Connecting the Distant Universe with the Local Fossil Record, Paris-Meudon Observatory. pp. 21-25, Sept. 1998. Dordrecht, The Netherlands: Kluwer. In press

Merrill PW. 1952. Science 115:484

Meyer BS. 1994. Annu. Rev. Astron. Astrophys. 32:153

Mould J, Reid N. 1987. Ap. J. 321:156

Mowlavi N, Jorissen A, Arnould M. 1996. Astron. Astrophys. 311:803

Mowlavi N, Jorissen A, Arnould M. 1998. Astron. Astrophys. 334:153

Murty SVS, Goswami JN, Shukolyukov A. 1997. Ap. J. 475:L65

Myers PC. 1998. Ap. J. 497:850

Nittler LR, Alexander CMO'D, Gao X, Walker RM, Zinner E. 1994. Nature 370:443

Nordlund A, Stein RS. 1995. In Stellar Evolution. What Should Be Done. XXXII Liège Astrophys. Colloq. ed. A Noels, D FraipontCaro, M Gabriel, N Grevesse, B Demarque, p. 75. Liège, Belgium: Inst. d'Astrophys.

Norris JE, Ryan SG, Beers T. 1997. Ap. J. 488: 350

North P, Berthet S, Lanz T. 1994. Astron. Astrophys. 281:775

Nyman LA, Olofsson H, Johansson LEB. 1993. Astron. Astrophys. 269:377

Olofsson H, Eriksson K, Gustafsson B, et al. 1993a. Ap. J. Suppl. 87:267

Olofsson H, Eriksson K, Gustafsson B, et al. 1993b. Ap. J. Suppl. 87:305

Ott U, Begemann F. 1990. Ap. J. 353:L57

Paczyński B. 1970. Acta Astron. 20:47

Paczyński B. 1971. Acta Astron. 21:417
Paczyński B. 1975. Ap. J. 202:558

Pilachowski CA. 1998. In Unsolved Problems in Stellar Evolution. Cambridge, U.K.: Cambridge Univ. Press. In press

Pilachowski CA, Sneden C, Hinkle K, Joyce R. 1997. Ap. J. 114:545

Plez B, Brett JM, Nordlund A. 1992. Astron. Astrophys. 256:551

Plez B, Smith VV, Lambert DL. 1993. Ap. J. 418:812

Podosek FA, Cassen P. 1994. Meteoritics 29:6

Podosek FA, Nichols RH Jr. 1997. In Proc. AIP Conf. 402, Astrophysical Implications Laboratory Study Presolar Materials, ed. TJ Bernatowicz, E Zinner, p. 617. Woodbury, NY: Am. Inst. Phys.

Prantzos N, Harissopulos S. eds. 1999. Nuclei in the Cosmos V. Paris: Ed. Frontières. 592 pp.

Prantzos N, Diehl R. 1996. Phys. Rep. 267:1

Prinzhofer A, Papanastassiou DA, Wasserburg GJ. 1989. Ap. J. 344:L81

Qian Y-Z, Vogel P, Wasserburg GJ. 1998. Ap. J. 506:868

Raiteri CM, Villata M, Gallino R, Busso M, Cravanzola A. 1999. Ap. J. 518:L91

Ramaty R, Kozlovsky B, Lingenfelter RE. 1996. Ap. J. 456:525

Reimers D. 1975. In Problems in Stellar Atmospheres and Envelopes, ed. B Baschek, H Kegel, G Traving, p. 229. Berlin: SpringerVerlag

Renzini A. 1977. In Advanced Stages in Stellar Evolution, ed. P Bouvier, A Maeder, p. 151. Sauverny, Switzerland: Geneva Obs.

Renzini A. 1984. In Observational Tests of the Stellar Evolution Theory, ed. A Maeder, I Renzini, p. 21. Dordrecht, The Netherlands: Reidel

Renzini A. 1987. Astron. Astrophys. 188:49

Renzini A, Fusi Pecci F. 1988. Annu. Rev. Astron. Astrophys. 26:199

Renzini A, Voli M. 1981. Astron. Astrophys. 94:175

Reynolds JH. 1960. Phys. Rev. Lett. 4:8

Ritossa C, García-Berro E, Iben I Jr. 1996. Ap. J. 460:489 
Rogers GJ, Iglesias CA. 1992. Ap. J. 412:752

Rolfs CE, Rodney WS. 1988. Cauldrons in the Cosmos. Chicago: Univ. Chicago Press $561 \mathrm{pp}$.

Rotaru M, Birck J-L, Allègre CJ. 1992. Nature 358:465

Rowe MW, Kuroda PK. 1965. J. Geophys. Res. 70:709

Russell SS, Srinivasan G, Huss GR, Wasserburg GJ, MacPherson GJ. 1996. Science 273:757

Ryan SG, Norris JE, Bessell MS. 1991. Astron. J. 102:303

Sackmann IJ, Boothroyd AI. 1991. Ap. J. 366: 529

Sackmann IJ, Boothroyd AI. 1992. Ap. J. 393: L21

Sahai R, Trauger JT, Watson AM, Stapelfeldt KR, Hester JJ, et al. 1998. Ap. J. 493:301

Sahijpal S, Goswami JN, Davis AM, Grossman L, Lewis RS. 1998. Nature 391:559

Sandage A, Schwarzschild M. 1952. Ap. J. 116: 463

Sanders RH. 1967. Ap. J. 150:971

Scalo JM, Ulrich RK. 1973. Ap. J. 183:151

Schramm DN, Wasserburg GJ. 1970. Ap. J. 162: 57

Schönberner D. 1979. Astron. Astrophys. 79: 108

Schönberner D. 1983. Ap. J. 272:708

Schröder K-P, Winters JM, Arndt TU, Sedlmayr E. 1998. Astron. Astrophys. 335:L9

Schwarzschild M, Härm R. 1965. Ap. J. 142:885

Schwarzschild, M, Härm R. 1967. Ap. J. 150: 961

Secchi A. 1868. C. R. Acad. Sci. Paris 66:124

Sedlmayr E, Dominik C. 1995. Space Sci. Rev. 73:211

Seeger PA, Fowler WA, Clayton DD. 1965. Ap. J. Suppl. 11:121

Sharp CM. 1992. Astron. Astrophys. Suppl. 94:1

Sharp CM, Wasserburg GJ. 1995. Geochim. Cosmochim. Acta 59:1632

Shaviv G, Salpeter EE. 1973. Ap. J. 184:191

Shukolyukov A, Lugmair GW. 1993a. Science 259:1138

Shukolyukov A, Lugmair GW. 1993b. Earth Planet. Sci. Lett. 119:159
Singh HP, Roxburgh IW, Chan KL. 1998. Astron. Astrophys. 340:178

Smith VV. 1984. Astron. Astrophys. 132:326

Smith VV, Lambert DL. 1985. Ap. J. 294:326

Smith VV, Lambert DL. 1986. Ap. J. 311:843

Smith VV, Lambert DL. 1989. Ap. J. 345:L75

Smith VV, Lambert DL. 1990. Ap. J. Suppl. 72: 387

Smith VV, Plez B, Lambert DL, Lubowich DA. 1995. Ap. J. 441:735

Smith VV, Wallerstein G. 1983. Ap. J. 273: 742

Sneden C, Cowan JJ, Burris DL, Truran JW. 1998. Ap. J. 496:235

Sneden C, Kraft R, Prosser CF, Langer GE. 1991. Astron. J. 102:2001

Sneden C, McWilliam A, Preston GW, Cowan JJ, Burris DL, Armosky BJ. 1996. Ap. J. 467: 819

Sneden C, Parthasarathy M. 1983. Ap. J. 267: 757

Srinivasan G, Sahijpal S, Ulyanov AA, Goswami GN. 1996. Geochim. Cosmochim. Acta 60:1823

Srinivasan G, Ulyanov AA, Goswami JN. 1994. Ap. J. 431:L67

Spite M, Spite F. 1991. Astron. Astrophys. 252: 689

Stewart B, Papanastassiou DA, Wasserburg GJ. 1996. Earth Planet. Sci. Lett. 143:1

Straniero O, Chieffi A, Limongi M, Busso M, Gallino R, Arlandini C. 1997. Ap. J. 478: 332

Straniero O, Gallino R, Busso M, Chieffi A, Raiteri CM, et al. 1995. Ap. J. 440:L85

Sugimoto D. 1971. Prog. Theor. Phys. 45:761

Sweigart AV, Gross PG. 1978. Ap. J. Suppl. 36: 405

Sweigart AV, Mengel JG. 1979. Ap. J. 229: 624

Takahashi K, Yokoi K. 1987. In At. Data Nucl. Data Tables 36:375

Travaglio C, Galli D, Gallino R, Busso M, Ferrini F, Straniero O. 1999. Ap. J. 520:

Truran JW. 1981. Astron. Astrophys. 97:371

Truran JW, Cameron AGW. 1978. Ap. J. 219: 226 
Truran JW, Iben I Jr. 1977. Ap. J. 216:797

Ulrich RK. 1973. In Explosive Nucleosynthesis, ed. DN Schramm, WD Arnett, p. 139. Austin: Univ. Texas Press

Utsumi K. 1985. In Cool Stars with Excesses of Heavy Elements, ed. M Jashek, PC Keenan, p. 243. Dordrecht, The Netherlands: Reidel

Vanhala HAT, Cameron AGW. 1998. Ap. J. 508: 291

van der Veen WECJ, Habing HJ. 1988. Astron. Astrophys. 194:125

van Loon JTH, Zijlstra AA, Whitelock PA, Te Linkel Hekkert P, Chapman JM, Loup C. 1998. Astron. Astrophys. 329:169

Vanture AD. 1992. Astron. J. 104:1986

Vassiliadis E, Wood PR. 1993. Ap. J. 413: 641

Voors RHM, Waters LBFM, Morris PW, Trams NR, DeKoter A, Bouwman J. 1999. Astron. Astrophys. 341:L67

Waelkens C, Van Winkel H, Boegaert E, Trams NR. 1991. Astron. Astrophys. 251: 495

Wagenhuber J, Groenewegen MAT. 1998. Astron. Astrophys. 340:183

Wallerstein G, Dominy JF. 1988. Ap. J. 330: 937

Wallerstein G, Iben I Jr, Parker P, Boesgaard AM, Hale GM, et al. 1997. Rev. Mod. Phys. 69:995

Wallerstein G, Knapp GR. 1998. Annu. Rev. Astron. Astrophys. 36:369

Ward RA. 1977. Ap. J. 216:540

Ward RA, Newman MJ. 1978. Ap. J. 219: 195

Ward RA, Newman MJ, Clayton DD. 1976. Ap. J. Suppl. 31:33

Wasserburg GJ. 1987. Earth Planet. Sci. Lett. $86: 129$
Wasserburg GJ, Boothroyd AI, Sackmann I-J. 1995. Ap. J. 447:L37

Wasserburg GJ, Busso M, Gallino R. 1996. Ap. J. 466:L109

Wasserburg GJ, Busso M, Gallino R, Raiteri CM. 1994. Ap. J. 424:412

Wasserburg GJ, Fowler WH, Hoyle F. 1960. Phys. Rev. Lett. 4:112

Wasserburg GJ, Gallino R, Busso M. 1998. Ap. J. 500:L189

Wasserburg GJ, Gallino R, Busso M, Goswami JN, Raiteri CM. 1995. Ap. J. 440:L101

Waters LBFM, Loup C, Kester DJM, Bontekoe TJR, de Jong T. 1994. Astron. Astrophys. 281: L1

Waters LBFM, Beintema DA, Zijlstra AA, De Kroter A, Molster FJ, et al. 1998. Astron. Astrophys. 331:L61

Weigert A. 1996. Z. Astrophys. 64:395

Wheeler CJ, Sneden C, Truran JW. 1989. Annu. Rev. Astron. Astrophys. 27:391

Willems FJ, de Jong T. 1988. Astron. Astrophys. 196:173

Winters RR, Macklin RL. 1982. Phys. Rev. C 25:208

Wood PR. 1981. In Physical Processes in Red Giants, ed. I Iben Jr., A Renzini, p. 135. Dordrecht, The Netherlands: Reidel

Woosley SE, Weaver TA. 1995. Ap. J. Suppl. 101:181

Wyse RFG, Silk J. 1987. Ap. J. 313:L11

Yamarura I, De Jong T, Onaka T, Cami J, Waters LBFM. 1998. Astron. Astrophys. 341:L9

Zahn JP. 1992. Astron. Astrophys. 265:115

Zinner E. 1997. In Proc. AIP Conf. 402, Astrophysical Implications Laboratory Study Presolar Materials, ed. TJ Bernatowicz, E Zinner, p. 3. St. Louis Mo. Woodbury, NY: Am. Inst. Phys. 
$12 \begin{aligned} & \text { Annual Review of Astronomy and Astrophysics } \\ & \text { Volume 37, } 1999\end{aligned}$

\section{CONTENTS}

Adventures in Cosmogony, A. G. W. Cameron 1

A Critical Review of Galactic Dynamos, Russell M. Kulsrud 37

Frequency Allocation: The First Forty Years, Brian Robinson 65

Reference Frames in Astronomy, K. J. Johnston and Chr. de Vegt 97

Probing the Universe with Weak Lensing, Yannick Mellier 127

The HR Diagram and the Galactic Distance Scale After Hipparcos, I.
Neill Reid

Nucleosynthesis in Asymptomatic Giant Branch Stars, M. Busso, R. 239

Gallino and G. J. Wasserburg

Physical Conditions in Regions of Star Formation, Neal J. Evans II 311

High-Energy Processes in Young Stellar Objects, Eric D. Feigelson and Thierry Montmerle

Sources of Relativistic Jets in the Galaxy, I. F. Mirabel and L. F. Rodríguez

409

The First 50 Years at Palomar: 1949-1999, Allan Sandage 445

Elemental Abundances in Quasistellar Objects, Fred Hamann and Gary 487

Ferland

Origin and Evolution of the Natural Satellites, S. J. Peale 533

Far-Ultraviolet Radiation from Elliptical Galaxies, Robert W. O'Connell 603 